\title{
UM PROBLEMA SEMILINEAR ELÍPTICO EM DOMÍNIO FINO COM TERMOS DE REAÇÕES CONCENTRADAS NA FRONTEIRA
}

\author{
Salvador Rafael Manjate \\ DisSERTAÇÃo APRESENTADA \\ AO \\ Instituto de Matemática e Estatística \\ DA \\ Universidade de São Paulo \\ PARA \\ OBTENÇÃO DO TÍTULO \\ DE \\ Mestre em CiênCias
}

Programa: Matemática

Orientador: Prof. Dr. Marcone Corrêa Pereira

Durante o desenvolvimento deste trabalho a autor recebeu auxílio financeiro do MCT-Moçambique.

São Paulo, 03 de Agosto de 2015. 

Comissão Julgadora:

- Prof. Dr. Marcone Corrêa Pereira (orientador) - IME-USP 



\section{AGRADECIMENTOS}

Para que este trabalho se tornasse realidade, houve colaboração de várias pessoas pelo que gostaria, antes de tudo, de agradecer pelo apoio prestado para sua realização:

Com muita satisfação ao Prof. Marcone Corrêa Pereira meu Orientador por ter me apresentado esse mundo de Equações Diferenciais Parciais em domínios finos, pelos ensinamentos, orientações constantes, incentivo e pela confiança diante de muitas provações na concepção e prosecussão deste trabalho.

A todos os professores e funcionários do Instituto de Matemática e Estatística (IME) da Universidade de São Paulo (USP) que me formaram e aqueles que manifestaram o seu apoio em especial aos Professores Vyacheslav Futorny, António Luiz Pereira e Eduardo de Nascimento, estendendo a minha gratidão a Professora Gleiciane Aragão da Universidade Federal de São Paulo (UNIFESP) por todas dicas e observações que nos deu durante a realização dos seminários no IME e em geral ao grupo da Dinâmica de Evolução do IME por cada palestra, seminários que serviram de muito aprendizado para todos nós.

Sem me esquecer dos meus companheiros das trincheiras André Porto, André Zaidan, Valdir Filho, Inocêncio Zimba, Arcelino Lobato, German Scobar entre outros pelas discussões e sugestões que me prestaram ao longo desses anos todos. Em especial a Ariadne Nogueira e Jean Nakasato pelo apoio tanto em material assim como em discussões durante os seminários e em geral a toda comunidade imeana.

Ao Departamento de Matemática e Informática (DMI) da Universidade Eduardo Mondlane (UEM) por ter permitido que a minha formação se tornasse uma realidade em especial aos Professores Manuel Alves, Andrei Shindiapin, João Munembe, Yuriy Nepomnyashchikh entre outros por terem tido um papel determinante para minha ida a formação. Aos meus colegas e amigos José Nhavoto, Betuel Canhanga, Domingos Djinja, Helvécio Lumenta, Manuel Nhangumbe, Américo Matusse, Timóteo Sambo, entre outros. 
Ao Ministério de Ciências e Tecnologias (MCT) Moçambique através da Direcção de Desenvolvimento e Capacitação dos Recursos Humanos (DDRCHCT) pelo suporte financeiro.

Por último, mas não os últimos, aos meus familiares o meu muito obrigado pelo apoio constante, paciência e colaboração que concederam ao longo de toda vida, o que impulsionou para que este trabalho pudesse ver a luz do dia. Sem me esquecer dos meus amigos Moçambique/Brasil e todos aqueles que directa ou indirectamente estiveram sempre comigo.

Finalmente à Laura Fernando pelo amor e carinho contínuo que tem me proporcionado e aos meus filhos Salésia e Salaciel, mesmo sabendo que nada pode recompensar o tempo que estive distante deles, mas sempre me deram força e serviram de inspiração para que pudesse concluir este curso. Obrigado! 


\section{RESUMO}

Neste trabalho analizamos o comportamento assintótico de uma família de equilíbrios de uma equação de reação-difusão com condição de fronteira de Neumann homogênea definida num domínio fino bidimensional com termos de reações concentradas em uma vizinhancca oscilante da fronteira. Assumimos que o domínio e portanto a vizinhança oscilante se degeneram em um intervalo quando um parâmetro positivo $\epsilon$ suficientemente pequeno tende para zero. O Objetivo principal deste trabalho é mostrar que essa família de soluções converge para a solução de uma equação limite, unidimensional, que captura a geometria e o comportamento assintótico dos conjuntos abertos onde o problema é estabelecido.

Palavras-chave: Equações semilineares elípticas, equações singulares elípticas, semi-continuidade superior, semi-continuidade inferior, domínios finos, termos concentrados. 


\section{ABSTRACT}

In this work we analyze the behaviour of a family of stead state solutions of a semilinear reaction-diffusion equation with homogeneous Neumann boundary condition, posed in a twodimensional thin domain with reaction terms concentrated in a narrow oscillating neighborhood. Indeed, we assume that the domain of definition of the solutions degenerates into an interval as a small parameter $\epsilon$ goes to zero. Our main result is that this family of solutions converge to the solution of a one-dimensional limit equation capturing the geometry and oscillatory behaviour of the open sets where the problem is estabilished.

Keywords: Semilinear elliptic equations, singular elliptic equations, upper semicontinuity, lower semicontinuity, thin domains, concentrating terms. 


\section{SUMÁRIO}

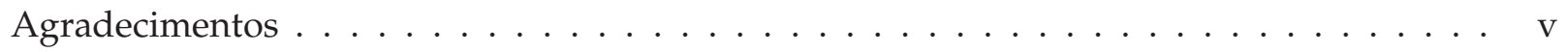

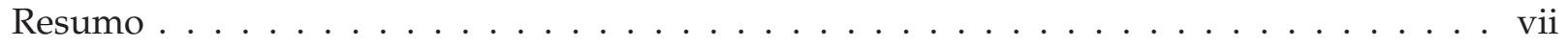

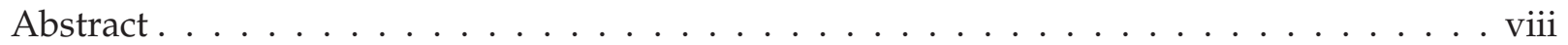

1 INTROduÇÃo 1

1.1 Contextualização . . . . . . . . . . . . . . . . . . . . 1

1.2 Colocação e descrição do problema . . . . . . . . . . . . . . . . . . 3

2 Integrais Concentradas $\quad 8$

2.1 Equivalência entre os Problemas $(1.4)$ e $(1.7) \ldots \ldots \ldots \ldots \ldots$

2.2 Colocação abstrata e a existência de soluções . . . . . . . . . . . . . . . . . . 12

2.2.1 Colocação abstrata e existência de soluções do problema (1.7) . . . . . . . . 12

2.2.2 Colocação abstrata e existência de soluções do problema (1.5) . . . . . . . . . . 18

2.3 Caracterização dos domínios de definição . . . . . . . . . . . . . . . . . . . 21

2.4 Integrais concentradas $\ldots \ldots \ldots \ldots \ldots \ldots \ldots \ldots$

3 SEMI-CONTINUIDADE SUPERIOR E INFERIOR DAS SOLUÇÕES 32

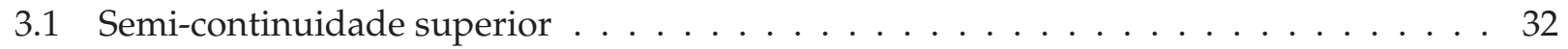

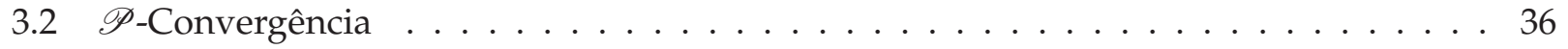

3.3 Propriedades das aplicações $F_{\epsilon} \ldots \ldots \ldots \ldots \ldots \ldots \ldots \ldots \ldots \ldots \ldots$

3.4 Conjunto de equilíbrios . . . . . . . . . . . . . . . . . 41

3.5 Semi-continuidade inferior . . . . . . . . . . . . . . . . . . . . 42 
A Considerações Gerais 45

A.1 Formas lineares em espaços de Banach . . . . . . . . . . . . . . . . . . . 45

A.2 Alguns resultados sobre espaços $L^{P} \ldots \ldots \ldots \ldots \ldots \ldots \ldots \ldots$

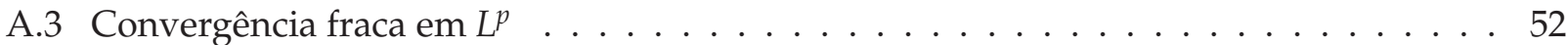

A.4 Funções periódicas rapidamente oscilantes . . . . . . . . . . . . . . . . . 54

A.4.1 Funções periódicas em $L^{1} \ldots \ldots \ldots \ldots \ldots \ldots$. . . . . . . . . . 54

B Alguns resultados sobre espaços de Sobolev 61

B.1 Derivada fraca . . . . . . . . . . . . . . . . . . . . . 61

B.2 Distribuições . . . . . . . . . . . . . . . . . . . . . . . 62

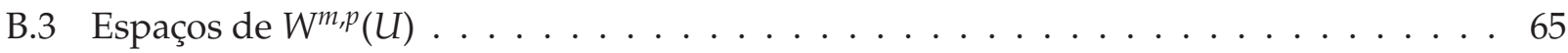

B.4 Imersões de Sobolev . . . . . . . . . . . . . . . . . . 66

B.5 Espaço $W_{0}^{m, p}(U) \ldots \ldots \ldots \ldots \ldots \ldots \ldots \ldots \ldots$

C Teorema de Lax-Milgram $\quad 72$

C.1 Formas bilineares em espaços de Banach . . . . . . . . . . . . . . . . . . . 72

C.2 Teorema de Lax-Milgram . . . . . . . . . . . . . . . . . . . 74

D Grau topológico de Leray \& Schauder

D.1 Aplicações Leray \& Schauder . . . . . . . . . . . . . . . . . . . . . . . . 75

D.2 Grau topológico de Leray \& Schauder . . . . . . . . . . . . . . . . . . 77

$\begin{array}{lr}\text { Referências bibliográficas } & 81\end{array}$ 


\section{CAPÍTULO 1}

\section{INTRODUÇÃO}

\subsection{Contextualização}

Neste trabalho analizamos o comportamento assintótico de uma família de soluções de uma equação parábolica semilinear, com condições de contorno Neumann homogêneas definida em um domínio fino $R^{\epsilon} \subset \mathbb{R}^{2}$, com termos de reações concentradas em uma vizinhança oscilante $\theta_{\epsilon}$ da fronteira superior. Mas precisamente, lidamos com um problema não-linear elíptico definido em um conjunto aberto de $\mathbb{R}^{2}$ que se degenera em um segmento de reta quando o parâmetro positivo $\epsilon$ tende para zero. Além disso, supomos que alguns termos de reação da equação ocorrem somente em uma região extremamente fina próxima da fronteira, região essa que também pode apresentar estrutura oscilatória.

Na figura 1.1 está ilustrado o domínio fino $R^{\epsilon}$, bem como a faixa oscilante $\theta_{\epsilon}$ onde tem lugar as reações concentradas

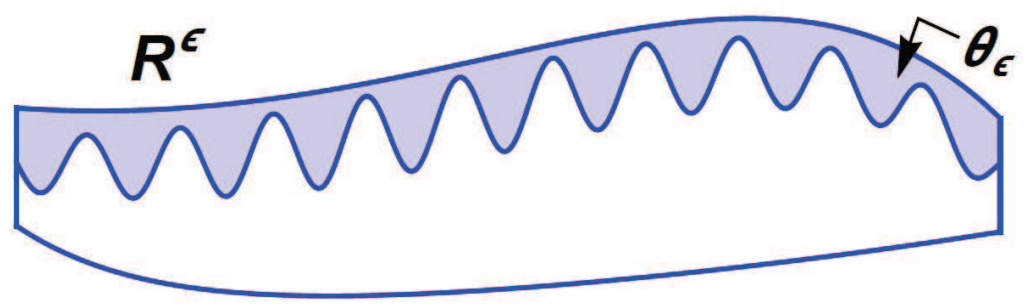

Figura 1.1: O domínio fino $R^{\epsilon}$ e a faixa oscilante $\theta_{\epsilon}$.

O problema de valor de contorno proposto pode ser entendido como um modelo de difusão e interação de agentes que podem ser células, quantidade de produtos químicos ou organismo 
biológicos, que estão localizadas numa região extremamente fina numa pequena vizinhança do bordo onde as reações podem ocorrer. Vale a pena notar que o nosso modelo inclui a possibilidade de que a faixa estreita apresente um comportamento oscilante modelando regiões de complexas interações. As aplicações potenciais dos nossos resultados incluem campos como os da lubrificação, nanotecnologia, de interação dinâmica vascular e gestão de controle de sistemas ecológicos e aquáticos onde pode-se encontrar concentrações localizadas em conexão com a fronteira complexa em canais finos.

Usando técnicas adequadas mostramos que o nosso problema singular definido em um conjunto aberto bidimensional pode ser aproximado por um problema de valor de fronteira unidimensional. Nesse caso a geometria do domínio fino e o comportamento oscilatório da faixa estreita onde ocorrem as reações são capturados pela equação limite unidimensional mostrando o efeito de tais variáveis no limite. A equação limite obtida é não singular, e nos dá uma aproximação do problema original quando o parâmetro $\epsilon$ está próximo de zero (sendo uma opção vantajosa, por exemplo, para a realização de simulações numéricas).

Existem vários trabalhos na literatura que lidam com Equações Diferenciais Parciais em domínios finos. Entre tantos, mencionamos os trabalhos pioneiros de J. Hale e G. Raugel [17, 11], bem como os artigos subsequentes [35, 30, 29], onde os autores investigaram o comportamento assintótico do sistema dinâmico dado por uma classe de equações semilineares parabólicos definidos em um domínio fino em $\mathbb{R}^{n}, n \geq 2$. Mencionamos também [33] onde o autor estudou as aproximações assintóticas das soluções do problema $p$-Laplaciano definido em uma região fina e [34] onde consideraram um problema linear elíptico em domínio perfurado com espessura rapidamente oscilante. Veja também $[3,4]$ que consideraram problemas monótonos não-lineares em multidomínios com fronteira rapidamente oscilante. Mencionamos também [18, 19, 20, 21, 24, 26, 27] onde estudaram uma classe de problemas elípticos e parabólicos com condição de contorno de Neumann homogênea discutindo-se questões ligadas aos problemas limites e propriedades de convergência.

Por outro lado, existem muitos trabalhos que lidam com problemas singulares elípticos e parabólicos que podem modelar fenômenos com termos potenciais e de reação concentrados numa pequena vizinhança de uma porção da fronteira de um domínio fixado e limitado. Nessa direção mencionamos os trabalhos $[1,2,15]$. Em $[8,9,25]$ os autores também estudaram esse mesmo tipo de problema permitindo comportamento oscilatório da faixa fina onde tais concentrações e potenciais são efetivos.

Nessa dissertação estudamos um modelo baseado em uma equação diferencial semiliniar do tipo elíptico que combina as duas situações descritas anteriormente de uma maneira mais geral. Para 
tanto observamos que é necessário adaptar as técnicas e métodos desenvolvidas em [17, 15, 9], bem como em [16], afim de passar o limite obtendo assim o comportamento assintótico do nosso modelo quando $\epsilon \rightarrow 0$.

\subsection{Colocação e descrição do problema}

Com o objetivo de introduzir nosso modelo consideramos inicialmente o domínio fino $R^{\epsilon} \subset \mathbb{R}^{2}$ definido por

$$
R^{\epsilon}=\left\{\left(x_{1}, x_{2}\right) \in \mathbb{R}^{2}: x_{1} \in(0,1),-\epsilon b\left(x_{1}\right)<x_{2}<\epsilon G\left(x_{1}\right)\right\},
$$

onde $G, b:(0,1) \rightarrow \mathbb{R}^{+}$são funções positivas, suaves e uniformemente limitadas, satisfazendo $0<G_{0} \leq G(x) \leq G_{1}$ e $0<b_{0} \leq b(x) \leq b_{1}$ para todo $x \in(0,1)$.

Observação 1.2.1 As funções Ge b são independentes de $\epsilon$ e definem a fronteira superior e inferior do domínio fino $R^{\epsilon}$.

A faixa oscilante $\theta_{\epsilon}$ é definida por

$$
\theta_{\epsilon}=\left\{\left(x_{1}, x_{2}\right) \in \mathbb{R}^{2}: x_{1} \in(0,1), \epsilon\left(G\left(x_{1}\right)-\epsilon^{\alpha} H_{\epsilon}\left(x_{1}\right)\right)<x_{2}<\epsilon G\left(x_{1}\right)\right\},
$$

onde $\alpha>0$ é um parâmetro, $H_{\epsilon}:(0,1) \rightarrow \mathbb{R}^{+}$é uma função suave, não negativa e uniformemente

limitada satisfazendo $0 \leq H_{\epsilon}(x) \leq G_{0}+b_{0} \stackrel{\text { def }}{=} H_{0}$. Como um dos objetivos é de modelar vizinhanças rugosas para concentrações, permitimos que $H_{\epsilon}$ oscile com respeito a variação parâmetro $\epsilon$. Para tanto a expressamos por

$$
H_{\epsilon}(x)=H\left(x, x / \epsilon^{\beta}\right), \quad \beta>0,
$$

onde $H:(0,1) \times \mathbb{R} \rightarrow \mathbb{R}$ é uma função suave, não negativa, limitada e periódica na segunda variável. Permitimos ainda que $H(x, \cdot)$ seja uma função periódica de período variável $l(x) \operatorname{com} x \in(0,1)$. (Mas detalhes sobre a função $H_{\epsilon}$ serão dadas na seção 2.3 ).

Observação 1.2.2 O conjunto aberto $\theta_{\epsilon}$ é uma vizinhança para a fronteira superior de $R^{\epsilon}$ cuja espessura e $O$ comportamento oscilatório dependem de $\alpha$ e $\beta$ respectivamente, bem como da periodicidade de $H$ na segunda variável. Dessa maneira os valores $\epsilon^{\alpha}$ e $\epsilon^{\beta}$ representam a ordem da espessura e a ordem de oscilação da vizinhança $\theta_{\epsilon}$ quando $\epsilon$ tende a zero.

Assim, vamos analizar a família de soluções definidas pelo seguinte problema semilinear elíptico

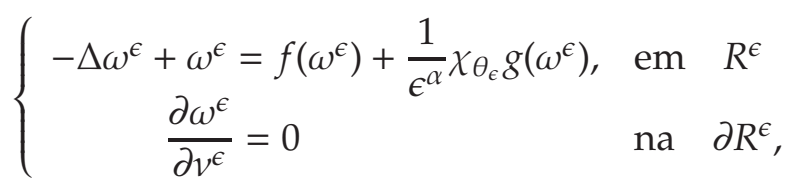


onde $v^{\epsilon}=\left(v_{1}^{\epsilon}, v_{2}^{\epsilon}\right)$ é o vetor unitário normal exterior à fronteira de $\partial R^{\epsilon} \mathrm{e} \frac{\partial}{\partial v^{\epsilon}}$ é a derivada normal exterior. As funções não lineares $f$ e $g: \mathbb{R} \rightarrow \mathbb{R}$ são funções da classe $C^{2}$, limitadas e com derivadas limitadas, e $\chi_{\theta_{\epsilon}}: \mathbb{R}^{2} \rightarrow \mathbb{R}$ é a função característica da faixa estreita $\theta_{\epsilon}$.

Procedendo de modo análogo a $[15,25]$ combinamos a função característica $\chi_{\epsilon}$, o parâmetro positivo $\epsilon$ e o valor fixado $\alpha$ para representar a concentração das reações na região $\theta_{\epsilon} \subset R^{\epsilon}$ quando $\epsilon$ tende para zero através do termo

$$
\frac{1}{\epsilon^{\alpha}} \chi_{\theta_{\epsilon}} \in L^{\infty}\left(R^{\epsilon}\right)
$$

Observemos ainda que $R^{\epsilon} \subset(0,1) \times\left(-\epsilon b_{1}, \epsilon G_{1}\right)$ é fino no sentido de que se degenera ao intervalo unitário quando $\epsilon \rightarrow 0$, razão pela qual espera-se que a família $\omega^{\epsilon}$ convirja para uma solução de uma equação unidimensional do mesmo tipo com a condição de fronteira de Neumann, capturando a geometria do contorno de $R^{\epsilon}$, bem como o comportamento oscilatório da faixa esteita $\theta_{\epsilon}$. Com efeito, sob estas condições mostra-se que o problema limite para (1.4) é dada por

$$
\left\{\begin{aligned}
-\frac{1}{p(x)}\left(p(x) \omega_{x}\right)_{x}+\omega & =f(\omega)+\frac{\mu(x)}{p(x)} g(\omega), \text { em } \quad(0,1) \\
\omega_{x}(0)=\omega_{x}(1) & =0
\end{aligned}\right.
$$

onde as funções suaves $p$ e $\mu:(0,1) \rightarrow(0, \infty)$ são definidas por

$$
p(x)=b(x)+G(x) \quad \mu(x)=\frac{1}{l(x)} \int_{0}^{l(x)} H(x, y) d y .
$$

$p$ está associado a geometria do domínio fino e é estabelecido pelas funções $b$ e $G$. A função $\mu \in L^{\infty}(0,1)$ é não negativa e está associada a faixa oscilante $\theta_{\epsilon}$ dada pela função $H_{\epsilon}$. Como foi mencionado, obtemos um problema limite que captura o perfil do domínio fino $R^{\epsilon}$ e o comportamento oscilante da faixa estreita $\theta_{\epsilon}$ combinando os resultados obtidos em $[9,11]$. Com efeito, aplicamos as técnicas de [17] para calcular o coeficiente $p$ estabelecido pelo domínio fino $R^{\epsilon}$, e usamos as integrais concentradas discutidas em $[1,9,15]$ para obtermos a função $\mu(x)$, que é o valor médio de $H(x, \cdot)$ para cada $x \in(0,1)$.

Notemos que $\mu$ captura o comportamento oscilatório e a geometria da faixa estreita onde as reações são concentradas. Se $H$ é independente da segunda variável, então a vizinhança estreita não possui comportamento oscilatório, e assim $\mu=H(x)$ em $(0,1)$ (veja a figura 1.2). Se tomarmos $H \equiv 0$, o problema (1.4) não apresenta reações concentradas em qualquer região, se reduzindo ao problema considerado em [11, 17]. 


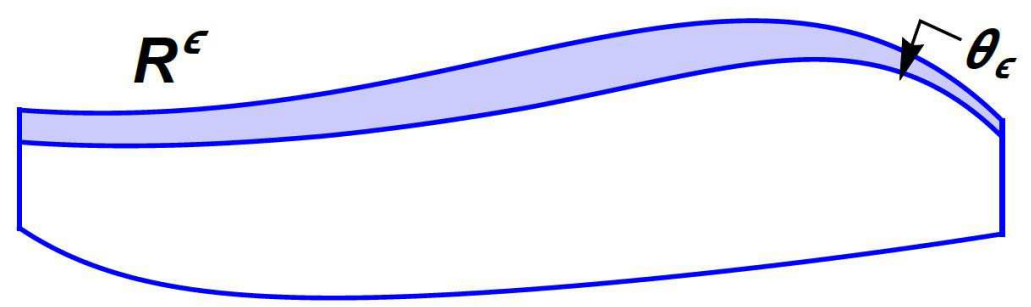

Figura 1.2: O domínio fino $R^{\epsilon}$ e a faixa não oscilante $\theta_{\epsilon}$.

Afim de estudar o problema (1.4) no domínio fino $R^{\epsilon}$ tomamos uma mudança de variável conveniente e obtemos o seguinte problema:

$$
\left\{\begin{array}{cc}
-\frac{\partial^{2} u^{\epsilon}}{\partial x_{1}^{2}}-\frac{1}{\epsilon^{2}} \frac{\partial^{2} u^{\epsilon}}{\partial x_{2}^{2}}+u^{\epsilon}=f\left(u^{\epsilon}\right)+\frac{1}{\epsilon^{\alpha}} \chi_{o_{\epsilon}} g\left(u^{\epsilon}\right), & \text { em } \quad \Omega \\
\frac{\partial u^{\epsilon}}{\partial x_{1}} N_{1}+\frac{1}{\epsilon^{2}} \frac{\partial u^{\epsilon}}{\partial x_{2}} N_{2}=0 & \text { na } \quad \partial \Omega,
\end{array}\right.
$$

onde $\Omega \subset \mathbb{R}^{2}$ é dado por

$$
\Omega=\left\{\left(x_{1}, x_{2}\right) \in \mathbb{R}^{2}: x_{1} \in(0,1),-b\left(x_{1}\right)<x_{2}<G\left(x_{1}\right)\right\}
$$

e $\chi_{o_{\epsilon}}: \mathbb{R}^{2} \rightarrow \mathbb{R}$ é a função característica da faixa estreita $o_{\epsilon}$ dada por

$$
o_{\epsilon}=\left\{\left(x_{1}, x_{2}\right) \in \mathbb{R}^{2}: x_{1} \in(0,1), G\left(x_{1}\right)-\epsilon^{\alpha} H_{\epsilon}\left(x_{1}\right)<x_{2}<G\left(x_{1}\right)\right\}
$$

$N=\left(N_{1}, N_{2}\right)$ é o vetor normal unitário exterior à $\partial \Omega$.

O fator $\epsilon^{-2}$ que aparece em frente da derivada parcial na direção de $x_{2}$ estabelece uma rápida difusão na direção vertical introduzindo um mecanismo de difusão muito forte. Nesse sentido redimensionamos a vizinhança $\theta_{\epsilon}$ para a faixa $o_{\epsilon} \subset \Omega$, e substituimos o domínio fino $R^{\epsilon}$ por um domínio que não é mais fino $\Omega$ e é independente de $\epsilon$.

Devido ao mecanismo de forte difusão espera-se que as soluções se tornem homogêneas na direção de $x_{2}$ de modo que a solução limite não varie nesta direção e por conseguinte o problema limitado será unidimensional. Isto está em concordância com a ideia intuitiva de que uma equação em domínio fino deve ser aproximada por uma equação definida num segmento de reta.

Agora já estamos em condições de enunciar o principal resultado deste trabalho.

Teorema 1.2.3 Seja $u^{\epsilon}$ uma família de soluções do problema (1.7) satisfazendo $\left\|u^{\epsilon}\right\|_{L^{\infty}(\Omega)} \leq R$ para alguma constante positiva independente de $\epsilon$. Então:

(i) Existe uma subsequência ainda denotada por $u^{\epsilon}$ e uma função $u \in H^{1}(\Omega)$, com $\|u\|_{L^{\infty}(\Omega)} \leq R$ dependendo apenas da primeira variável, isto é, $u\left(x_{1}, x_{2}\right)=u\left(x_{1}\right)$ solução do problema (1.5) satisfazendo

$$
\left\|u^{\epsilon}-u\right\|_{H^{1}(\Omega)} \rightarrow 0, \text { quando } \quad \epsilon \rightarrow 0 .
$$


(ii) Se a solução u de (1.5) pertence a uma bola de raio $R$ em $L^{\infty}(\Omega)$ e é hiperbólica, então também temos que existe uma sequência $u^{\epsilon}$ de soluções do problema (1.7) satisfazendo

$$
\left\|u^{\epsilon}-u\right\|_{H^{1}(\Omega)} \rightarrow 0 \text {, quando } \epsilon \rightarrow 0 .
$$

Observação 1.2.4 Dizemos que a solução u de um problema de valor de fronteira é hiperbólico se $\lambda=0$ não é autovalor do problema linearizado em torno de u. Por exemplo se u satisfaz a equação (1.5) e é hiperbólico, então $\lambda=0$ não é autovalor do problema de autovalor

$$
\left\{\begin{aligned}
-\frac{1}{p(x)}\left(p(x) v_{x}\right)_{x}+v & =f_{u}(u) v+\frac{\mu(x)}{p(x)} g_{u}(u) v+\lambda v, \text { em } \quad(0,1) \\
v_{x}(0)=v_{x}(1) & =0 .
\end{aligned}\right.
$$

Observação 1.2.5 Como estamos interessados em estudar soluções que são uniformemente limitadas em $L^{\infty}(\Omega)$, assumimos sem perda de generalidade que as funções não lineares $f$ e g são de classe $C^{2}$ com derivadas limitadas. Com efeito os valores assumidos por $f$ e $g$ fora da região $|u| \leq R$ não interferem no problema e assim, podem ser modificadas de maneira que o tratamento seja simplificado.

Observação 1.2.6 Denotemos por $\mathcal{E}_{\epsilon}$ o conjunto $\mathcal{E}_{\epsilon}=\left\{u^{\epsilon} \in H^{1}(\Omega): u^{\epsilon}\right.$ é solução de (1.7) $\}$. Se $\left\|u^{\epsilon}\right\|_{H^{1}(\Omega)} \leq$ $R$ sempre que $u^{\epsilon} \in \mathcal{E}_{\epsilon}$, (i) e (ii) no Teorema 1.2.3 respectivamente implicam na semi-continuidade superior $e$ inferior do conjunto de equilibrios do problema parabólico associado a (1.7) em $\epsilon=0$.

O presente trabalho é constituido por três capítulos, quatro apêndices e referências bibliográficas. O primeiro capítulo correspondente a Introdução. Nele faz-se a contextualização, colocação e descrição do problema. Definem-se os objetivos, e enuncia-se o resultado principal.

No segundo capítulo consideramos a Integrais concentradas, que são importantes para a passagem ao limite do problema (1.7). Também mostramos que os problemas (1.7) e (1.5) são equivalentes. Definimos formalmente os operadores ilimitados $\mathcal{A}_{\epsilon}$ e $\mathcal{A}_{0}$ e com base neles escrevemos os problemas (1.7) e (1.5) na forma abstrata e discutimos a existência de soluções. Introduzimos também as hipóteses gerais que norteam os dominios $R^{\epsilon}$ e $\Omega$ e as faixas $\theta_{\epsilon}$ e $o_{\epsilon}$. Finalmente, adaptamos os resultados obtidos em $[15,1,9]$ mostrando como integrais concentradas convergem para integrais de contorno.

O terceiro capítulo corresponde a semi-continuidade superior e inferior das soluções. Nele mostramos que soluções do problema (1.5) podem ser obtidas como limite do problema (1.7) mas precisamente a Proposição 3.1.1.

No Apêndice A fazemos uma breve revisão de algumas propriedades dos espaços $L^{p}(1 \leq p \leq \infty)$ e também apresentamos a demonstração do Teorema A.4.4 que é muito importante na obtenção dos 
nossos resultados, bem como na Teoria de Homogeneização. Formalmente, esse teorema diz que uma função periódica rapidamente oscilante $f_{\epsilon}(x)$ converge no sentido fraco para o seu valor médio $\mathcal{M}_{Y}(f)$ quando o número de oscilações aumenta.

No Apêndice B fazemos uma breve revisão sobre os espaços de Sobolev $W^{m, p}(U)$, como são construidos e algumas propriedades desses espaços, os Teorema de Imersão de Sobolev e de RellichKondravich que são frequentemente usados ao longo do texto.

No Apêndice $C$ fazemos uma breve revisão de formas bilineares nos espaços de Banach e o Teorema de Lax-Milgram.

No Apêndice D fazemos uma breve revisão sobre o grau topológico de Leray \& Schauder, devido a sua relevância apresentamos algumas propriedades das aplicações Leray \& Schauder e anunciamos alguns resultados sobre aplicações que são bastante úteis no estudo da semi-continuidade inferior. 


\section{CAPÍTULO 2}

\section{INTEGRAIS CONCENTRADAS}

Neste capítulo pretendemos mostrar a equivalência entre os problemas (1.4) e (1.7), dar a formulação abstrata dos problemas (1.7) e (1.5) e discutir a existência de soluções.Também vamos introduzir as hipóteses sobre caracterização dos domínios de definição das soluções mostrando finalmente como diferentes integrais concentradas convergem para integrais de superficie adaptando os resultados obtidos em $[15,1,9]$.

\subsection{Equivalência entre os Problemas (1.4) e (1.7)}

Afim de estudar o problema (1.4) no domínio fino $R^{\epsilon}$ fazemos uma mudança conveniente de variável que consiste em escalar o domínio fino $R^{\epsilon}$ por um domínio fixo $\Omega$, bem como a faixa fina $\theta_{\epsilon}$ por $o_{\epsilon} \subset \Omega$. Mas precisamente temos a seguinte proposição relacionada

Proposição 2.1.1 $\omega^{\epsilon} \in H^{1}\left(R^{\epsilon}\right)$ é solução do problema (1.4) se e só se, $u^{\epsilon} \in H^{1}(\Omega)$ é solução do problema (1.7).

Demonstração. Para provar esta proposição vamos proceder da seguinte maneira:

Passo 1. Escrever as formulações fracas dos problemas (1.4) e (1.7). No problema (1.4) denotemos $f\left(\omega^{\epsilon}\right)+\frac{1}{\epsilon^{\alpha}} \chi_{\theta_{\epsilon}} g\left(\omega^{\epsilon}\right)$ por $F^{\epsilon}\left(\omega^{\epsilon}\right)$, isto é, $F^{\epsilon}\left(\omega^{\epsilon}\right)=f\left(\omega^{\epsilon}\right)+\frac{1}{\epsilon^{\alpha}} \chi_{\theta_{\epsilon}} g\left(\omega^{\epsilon}\right)$ e reescrevermos o problema (1.4) como

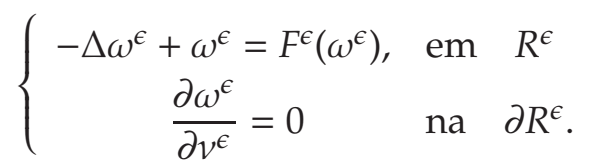

Temos que $\omega^{\epsilon} \in H^{1}\left(R^{\epsilon}\right)$ é solução para o problema (1.4) se e somente se satisfaz

$$
\int_{R^{\epsilon}} \nabla \omega^{\epsilon} \nabla \varphi^{\epsilon} d x_{1} d x_{2}+\int_{R^{\epsilon}} \omega^{\epsilon} \varphi^{\epsilon} d x_{1} d x_{2}=\int_{R^{\epsilon}} F_{\epsilon}\left(\omega^{\epsilon}\right) \varphi^{\epsilon} d x_{1} d x_{2}, \quad \forall \varphi^{\epsilon} \in H^{1}\left(R^{\epsilon}\right)
$$


Analogamente, denotando $F_{\epsilon}\left(u^{\epsilon}\right)=f\left(u^{\epsilon}\right)+\frac{1}{\epsilon^{\alpha}} \chi_{o_{\epsilon}} g\left(u^{\epsilon} 0\right)$, temos que $u^{\epsilon} \in H^{1}(\Omega)$ é solução do problema (1.7) se e somente se satisfaz

$$
\int_{\Omega}\left\{\frac{\partial u^{\epsilon}}{\partial x} \frac{\partial \psi}{\partial x}+\frac{1}{\epsilon^{2}} \frac{\partial u^{\epsilon}}{\partial y} \frac{\partial \psi}{\partial y}+u^{\epsilon} \psi\right\} d y d x=\int_{\Omega} F_{\epsilon}\left(u^{\epsilon}\right) \psi d y d x \quad \forall \psi \in H^{1}(\Omega) .
$$

Passo 2. Mostrar a equivalência das formulações fracas (2.1) e (2.2).

Afim de estudar o problema (2.1) definimos a seguinte aplicação

$$
\begin{aligned}
\phi: R^{\epsilon} & \rightarrow \Omega \\
\left(x_{1}, x_{2}\right) & \mapsto\left(x_{1}, \epsilon^{-1} x_{2}\right)=(x, y),
\end{aligned}
$$

onde $R^{\epsilon}$ e $\Omega$ são abertos introduzidos em (1.1) e (1.8). Notemos que $\phi\left(R^{\epsilon}\right)=\Omega$ e possui a matriz jacobiana $J_{\phi}\left(x_{1}, x_{2}\right)$ dado por

$$
J_{\phi}\left(x_{1}, x_{2}\right)=\left(\begin{array}{ll}
\frac{\partial x}{\partial x_{1}} & \frac{\partial x}{\partial x_{2}} \\
\frac{\partial y}{\partial x_{1}} & \frac{\partial y}{\partial x_{2}}
\end{array}\right)=\left(\begin{array}{cc}
1 & 0 \\
0 & \frac{1}{\epsilon}
\end{array}\right) .
$$

Não é difícil ver que $\phi$ é um defeomorfismo pelo Teorema da Aplicação inversa [37] e pelo fato de ser uma aplicação bijetora. Assim dado $\omega^{\epsilon}$ definido em $R^{\epsilon}$ definimos $u^{\epsilon}$ em $\Omega$ por

$$
u^{\epsilon}(x, y)=\omega^{\epsilon} \circ \phi^{-1}(x, y)
$$

Logo, $\omega^{\epsilon}\left(x_{1}, x_{2}\right)=u^{\epsilon} \circ \phi\left(x_{1}, x_{2}\right)=u^{\epsilon}\left(\phi\left(x_{1}, x_{2}\right)\right)=u^{\epsilon}(x, y)$, donde vemos que

$$
\frac{\partial \omega^{\epsilon}}{\partial x_{1}}\left(x_{1}, x_{2}\right)=\frac{\partial}{\partial x_{1}}\left(u^{\epsilon}\left(\phi\left(x_{1}, x_{2}\right)\right)\right)=\partial_{1}\left(u^{\epsilon}(x, y)\right) \frac{\partial x}{\partial x_{1}}+\partial_{1}\left(u^{\epsilon}(x, y)\right) \frac{\partial x}{\partial x_{2}}=\frac{\partial u^{\epsilon}}{\partial x}(x, y)
$$

e

$$
\frac{\partial \omega^{\epsilon}}{\partial x_{2}}\left(x_{1}, x_{2}\right)=\frac{\partial}{\partial x_{2}}\left(u^{\epsilon}\left(\phi\left(x_{1}, x_{2}\right)\right)\right)=\partial_{2}\left(u^{\epsilon}(x, y)\right) \frac{\partial y}{\partial x_{1}}+\partial_{2}\left(u^{\epsilon}(x, y)\right) \frac{\partial y}{\partial x_{2}}=\frac{1}{\epsilon} \frac{\partial u^{\epsilon}}{\partial x}(x, y)
$$

Analogamente, $\psi(x, y)=\varphi^{\epsilon} \circ \psi^{-1}(x, y)$ donde, $\frac{\partial \varphi^{\epsilon}}{\partial x_{1}}\left(x_{1}, x_{2}\right)=\frac{\partial \psi}{\partial x}(x, y)$ e $\frac{\partial \varphi^{\epsilon}}{\partial x_{1}}\left(x_{1}, x_{2}\right)=\frac{1}{\epsilon} \frac{\partial \psi}{\partial y}(x, y)$.

Agora em (2.1) aplicando o Teorema de Mudança de Variável [37] temos que (2.1) é equivalente à

$$
\int_{\Omega}\left\{\frac{\partial u^{\epsilon}}{\partial x} \frac{\partial \psi}{\partial x}+\frac{1}{\epsilon^{2}} \frac{\partial u^{\epsilon}}{\partial y} \frac{\partial \psi}{\partial y}+u^{\epsilon} \psi\right\} d y d x=\int_{\Omega} F_{\epsilon}\left(u^{\epsilon}\right) \psi d y d x \quad \forall \psi \in H^{1}(\Omega) .
$$

Passo 3. Mostrar a regularidade das soluções fracas do problema (2.3). Isto é, seja $u^{\epsilon} \in H^{1}(\Omega)$ satisfazendo (2.3) então $u^{\epsilon} \in H^{2}(\Omega)$.

De fato, $u^{\epsilon} \in H^{1}(\Omega)$ satisfaz (2.3),

$$
\begin{aligned}
\int_{\Omega}\left\{\frac{\partial u^{\epsilon}}{\partial x} \frac{\partial \psi}{\partial x}+\frac{1}{\epsilon^{2}} \frac{\partial u^{\epsilon}}{\partial y} \frac{\partial \psi}{\partial y}\right\} d y d x & =\int_{\Omega} f\left(u^{\epsilon}\right) \psi d y d x+\frac{1}{\epsilon^{\alpha}} \int_{o_{\epsilon}} g\left(u^{\epsilon}\right) \psi d y d x \\
& =\int_{\Omega}\left(f\left(u^{\epsilon}\right)+\frac{1}{\epsilon^{\alpha}} \chi_{o_{\epsilon}} g\left(u^{\epsilon}\right)\right) \psi d y d x=\int_{\Omega} F_{\epsilon}\left(u^{\epsilon}\right) \psi d y d x,
\end{aligned}
$$


para toda $\psi \in H^{1}(\Omega)$. Como $\Omega$ é um domínio Lipschtz contínua (Veja no Apêndice a Definição B.4.3), pelo Teorema de Grisvard sobre a regularidade de soluções fracas do problema de Neumann [31, Teoremas 3.2.1.3] temos que se $F_{\epsilon} \in L^{2}(\Omega)$ então $u \in H^{2}(\Omega)$ e satisfaz (2.3) com efeito,

$$
\begin{aligned}
\left\|F_{\epsilon}\right\|_{L^{2}(\Omega)}^{2}=\int_{\Omega}\left|f\left(u^{\epsilon}\right)+\frac{1}{\epsilon^{\alpha}} \chi_{o_{\epsilon}} g\left(u^{\epsilon}\right)\right|^{2} d y d x & \leq 2 \int_{\Omega}\left(\left|f\left(u^{\epsilon}\right)\right|^{2}+\frac{1}{\epsilon^{\alpha}}\left|\chi_{o_{\epsilon}} g\left(u^{\epsilon}\right)\right|^{2}\right) d y d x \\
& \leq 2\|f\|_{L^{\infty}(\mathbb{R})}^{2} \int_{\Omega} d y d x+2\|g\|_{L^{\infty}(\mathbb{R})}^{2} \int_{o_{\epsilon}} \frac{1}{\epsilon^{\alpha}} d y d x \\
& \leq 2\left(\|f\|_{L^{\infty}(\mathbb{R})}^{2}|\Omega|+\|g\|_{L^{\infty}(\mathbb{R})}^{2} H_{1}\right) .
\end{aligned}
$$

Passo 4. Concluir que os problemas (1.4) e (1.7) são equivalentes.

Com base no Passo 3. podemos obter o problema clássico cuja formulação fraca é dada por (2.3) que é equivalente a formulação fraca (2.1) pelo Passo 2.. Para tal consideremos o campo $X(x, y)$ dado por $X(x, y)=\left(\begin{array}{c}\frac{\partial u^{\epsilon}}{\partial x} \\ \frac{1}{\epsilon^{2}} \frac{\partial u^{\epsilon}}{\partial y}\end{array}\right)$ e $\psi=\psi(x, y)$. Devido ao Teorema de Green (Teorema B.5.4) temos que

$$
\int_{\partial \Omega} \psi X N=\int_{\Omega} \operatorname{div}(\psi X)=\int_{\Omega} \nabla \psi X+\int_{\Omega} \psi \operatorname{div}(X) .
$$

Então,

$$
\int_{\partial \Omega} \psi\left\{\frac{\partial u^{\epsilon}}{\partial x} N_{1}+\frac{1}{\epsilon^{2}} \frac{\partial u^{\epsilon}}{\partial y} N_{2}\right\} d S=\int_{\Omega}\left\{\frac{\partial u^{\epsilon}}{\partial x} \frac{\partial \psi}{\partial x}+\frac{1}{\epsilon^{2}} \frac{\partial u^{\epsilon}}{\partial y} \frac{\partial \psi}{\partial y}\right\} d y d x+\int_{\Omega}\left\{\frac{\partial^{2} u^{\epsilon}}{\partial x^{2}}+\frac{1}{\epsilon^{2}} \frac{\partial^{2} u^{\epsilon}}{\partial y^{2}}\right\} \psi d y d x,
$$

donde usando (2.3) temos

$$
\int_{\Omega}\left\{-\frac{\partial^{2} u^{\epsilon}}{\partial x^{2}}-\frac{1}{\epsilon^{2}} \frac{\partial^{2} u^{\epsilon}}{\partial y^{2}}+u^{\epsilon}-F_{\epsilon}\left(u^{\epsilon}\right)\right\} \psi d y d x+\int_{\partial \Omega} \psi\left\{\frac{\partial u^{\epsilon}}{\partial x} N_{1}+\frac{1}{\epsilon^{2}} \frac{\partial u^{\epsilon}}{\partial y} N_{2}\right\} d S=0,
$$

para toda $\psi \in H^{1}(\Omega)$. Notemos também que

$$
\begin{aligned}
\frac{\partial \omega^{\epsilon}}{\partial \nu^{\epsilon}}=\left\langle\nabla \omega^{\epsilon}, v^{\epsilon}\right\rangle & =\left[\frac{\partial \omega^{\epsilon}}{\partial x_{1}} \cdot\left(-\epsilon G^{\prime}\left(x_{1}\right)\right)+\frac{\partial \omega^{\epsilon}}{\partial x_{2}} \cdot 1\right]\left(1+\epsilon^{2} G^{\prime 2}\left(x_{1}\right)\right)^{-1 / 2} \\
& =\left[\frac{\partial \omega^{\epsilon}}{\partial x_{1}} \frac{-G^{\prime}\left(x_{1}\right)}{\sqrt{1+G^{\prime 2}\left(x_{1}\right)}}+\frac{1}{\epsilon} \frac{\partial \omega^{\epsilon}}{\partial x_{2}} \frac{1}{\sqrt{1+G^{\prime 2}\left(x_{1}\right)}}\right] \epsilon \sqrt{\frac{1+G^{\prime 2}\left(x_{1}\right)}{1+\epsilon^{2} G^{\prime 2}\left(x_{1}\right)}} \\
& =\left[\frac{\partial u^{\epsilon}}{\partial x} \frac{-G^{\prime}\left(x_{1}\right)}{\sqrt{1+G^{\prime 2}\left(x_{1}\right)}}+\frac{1}{\epsilon^{2}} \frac{\partial u^{\epsilon}}{\partial y} \frac{1}{\sqrt{1+G^{\prime 2}\left(x_{1}\right)}}\right] \epsilon \sqrt{\frac{1+G^{\prime 2}\left(x_{1}\right)}{1+\epsilon^{2} G^{\prime 2}\left(x_{1}\right)}} \\
& =\left(\frac{\partial u^{\epsilon}}{\partial x} N_{1}+\frac{1}{\epsilon^{2}} \frac{\partial u^{\epsilon}}{\partial y} N_{2}\right) \epsilon \sqrt{\frac{1+G^{\prime 2}\left(x_{1}\right)}{1+\epsilon^{2} G^{\prime 2}\left(x_{1}\right)}}
\end{aligned}
$$

Finalmente concluimos a prova da proposição tomando $\psi \in C_{c}^{\infty}(\Omega)$ o que implica que de (2.4) que $-\frac{\partial^{2} u^{\epsilon}}{\partial x^{2}}-\frac{1}{\epsilon^{2}} \frac{\partial^{2} u^{\epsilon}}{\partial y^{2}}+u^{\epsilon}=F_{\epsilon}\left(u^{\epsilon}\right)$ em $\Omega$ e se a $\psi$ se anula no interior de $\Omega$ de (2.4) implica que 
$\frac{\partial u^{\epsilon}}{\partial x} N_{1}+\frac{1}{\epsilon^{2}} \frac{\partial u^{\epsilon}}{\partial y} N_{2}=0$ em $\partial \Omega$, donde resulta o problema (1.7), ou seja

$$
\begin{cases}-\frac{\partial^{2} u^{\epsilon}}{\partial x_{1}^{2}}-\frac{1}{\epsilon^{2}} \frac{\partial^{2} u^{\epsilon}}{\partial x_{2}^{2}}+u^{\epsilon}=f\left(u^{\epsilon}\right)+\frac{1}{\epsilon^{\alpha}} \chi_{o_{\epsilon}} g\left(u^{\epsilon}\right), & \text { em } \quad \Omega \\ \frac{\partial u^{\epsilon}}{\partial x_{1}} N_{1}+\frac{1}{\epsilon^{2}} \frac{\partial^{\epsilon}}{\partial x_{2}} N_{2}=0 & \text { na } \partial \Omega,\end{cases}
$$

onde $N=\left(N_{1}, N_{2}\right)$ é o vetor normal exterior a $\partial \Omega$.

Denotaremos por $H_{\epsilon}^{1}(U)$ o espaço de Hilbert $H^{1}(U)$ dotado da norma

$$
\|w\|_{H_{\epsilon}^{1}(U)}=\left(\int_{U}\left|\frac{\partial w}{\partial x}\right|^{2} d y d x+\frac{1}{\epsilon^{2}} \int_{U}\left|\frac{\partial w}{\partial y}\right|^{2} d y d x+\int_{U}|w|^{2} d y d x\right)^{1 / 2}
$$

definido pelo produto interno

$$
(\phi, \varphi)_{H_{\epsilon}^{1}(U)}=\int_{U}\left\{\frac{\partial \phi}{\partial x} \frac{\partial \varphi}{\partial x}+\frac{1}{\epsilon^{2}} \frac{\partial \phi}{\partial y} \frac{\partial \varphi}{\partial y}+\phi \varphi\right\} d y d x
$$

onde $U$ é um aberto de $\mathbb{R}^{2}$.

Observação 2.1.2 Para todo $\epsilon \in(0,1)$ temos $\|\cdot\|_{H^{1}(\Omega)} \leq\|\cdot\|_{H_{\epsilon}^{1}(\Omega)}$.

Afirmação 2.1.3 Para cada $\epsilon>0$ fixado temos que as normas $\|\cdot\|_{H_{\epsilon}^{1}(\Omega)} e\|\cdot\|_{H^{1}(\Omega)}$ são equivalentes.

Prova. Para provar esta afirmacação vamos dividir em dois casos:

(1) Para $\epsilon \in(0,1)$ segue da Observação 2.1.2 que $\|\cdot\|_{H^{1}(\Omega)} \leq\|\cdot\|_{H_{\epsilon}^{1}(\Omega)}$. Por outro lado para todo $u \in H_{\epsilon}^{1}(\Omega)$ temos

$$
\begin{aligned}
\|u\|_{H_{\epsilon}^{1}(\Omega)}^{2} & =\int_{\Omega}\left\{\left(\frac{\partial u}{\partial x}\right)^{2}+\frac{1}{\epsilon^{2}}\left(\frac{\partial u}{\partial y}\right)^{2}+u^{2}\right\} d y d x=\frac{1}{\epsilon^{2}} \int_{\Omega}\left\{\epsilon^{2}\left(\frac{\partial u}{\partial x}\right)^{2}+\left(\frac{\partial u}{\partial y}\right)^{2}+\epsilon^{2} u^{2}\right\} d y d x \\
& \leq \frac{1}{\epsilon^{2}} \int_{\Omega}\left\{\left(\frac{\partial u}{\partial x}\right)^{2}+\left(\frac{\partial u}{\partial y}\right)^{2}+u^{2}\right\} d y d x=\frac{1}{\epsilon^{2}}\|u\|_{H^{1}(\Omega)}^{2} .
\end{aligned}
$$

Donde segue que $\|\cdot\|_{H^{1}(\Omega)} \leq\|\cdot\|_{H_{\epsilon}^{1}(\Omega)} \leq M_{\epsilon}\|\cdot\|_{H^{1}(\Omega)}$ onde $M_{\epsilon}:=\epsilon^{-1}$.

(2) Para $\epsilon>1$ temos que $\epsilon^{-2}<1$ portanto $\|\cdot\|_{H^{1}(\Omega)} \geq\|\cdot\|_{H_{\epsilon}^{1}(\Omega)}$. Por outro lado para cada $u \in H_{\epsilon}^{1}(\Omega)$ temos

$$
\begin{aligned}
\|u\|_{H_{\epsilon}^{1}(\Omega)}^{2} & =\int_{\Omega}\left\{\left(\frac{\partial u}{\partial x}\right)^{2}+\frac{1}{\epsilon^{2}}\left(\frac{\partial u}{\partial y}\right)^{2}+u^{2}\right\} d y d x=\frac{1}{\epsilon^{2}} \int_{\Omega}\left\{\epsilon^{2}\left(\frac{\partial u}{\partial x}\right)^{2}+\left(\frac{\partial u}{\partial y}\right)^{2}+\epsilon^{2} u^{2}\right\} d y d x \\
& \geq \frac{1}{\epsilon^{2}} \int_{\Omega}\left\{\left(\frac{\partial u}{\partial x}\right)^{2}+\left(\frac{\partial u}{\partial y}\right)^{2}+u^{2}\right\} d y d x=\frac{1}{\epsilon^{2}}\|u\|_{H^{1}(\Omega)}^{2} .
\end{aligned}
$$

Donde segue que $m_{\epsilon}\|\cdot\|_{H^{1}(\Omega)} \leq\|\cdot\|_{H_{\epsilon}^{1}(\Omega)} \leq\|\cdot\|_{H^{1}(\Omega)}$ onde $m_{\epsilon}:=\epsilon^{-1}$. 
Notemos que o espaço $H_{\epsilon}^{1}(\Omega)$ é adequado para trabalhar com problemas em domínio fino devido ao mecanismo de alta difusão que aparece em frente da segunda derivada na equação.

Observação 2.1.4 Resulta de (2.5) que se a sequência $u^{\epsilon} \in H_{\epsilon}^{1}(U)$ satisfaz $\left\|u^{\epsilon}\right\|_{H_{\epsilon}^{1}(U)} \leq K$ uniformemente em $\epsilon$, para alguma constante K independentemente de $\epsilon$, então

$$
\left\|\frac{\partial u^{\epsilon}}{\partial y}\right\|_{L^{2}(U)} \leq \epsilon K, \quad \forall \epsilon>0,
$$

e assim

$$
\left\|\frac{\partial u^{\epsilon}}{\partial y}\right\|_{L^{2}(\Omega)} \rightarrow 0, \quad \epsilon \rightarrow 0
$$

De fato,

temos que $H_{\epsilon}^{1}(\Omega)=\left\{u^{\epsilon} \in H^{1}(U): \int_{U}\left\{\left|\frac{\partial u^{\epsilon}}{\partial x}\right|^{2}+\frac{1}{\epsilon^{2}}\left|\frac{\partial u^{\epsilon}}{\partial y}\right|^{2}+\left|u^{\epsilon}\right|^{2}\right\} d y d x<\infty\right\}$. Assim como a sequência $u^{\epsilon} \in H_{\epsilon}^{1}(U)$ é tal que $\left\|u^{\epsilon}\right\|_{H_{\epsilon}^{1}(U)} \leq K$ uniformemente em $\epsilon$, então

$$
\left\|u^{\epsilon}\right\|_{H_{\epsilon}^{1}(U)}^{2}=\left\|u^{\epsilon}\right\|_{L^{2}(U)}^{2}+\left\|\frac{\partial u^{\epsilon}}{\partial x}\right\|_{L^{2}(U)}^{2}+\frac{1}{\epsilon^{2}}\left\|\frac{\partial u^{\epsilon}}{\partial y}\right\|_{L^{2}(U)}^{2} \leq K^{2}
$$

portanto

$$
\frac{1}{\epsilon^{2}}\left\|\frac{\partial u^{\epsilon}}{\partial y}\right\|_{L^{2}(U)}^{2} \leq\left\|u^{\epsilon}\right\|_{H_{\epsilon}^{1}(U)}^{2} \leq K^{2}
$$

donde,

$$
\left\|\frac{\partial u^{\epsilon}}{\partial y}\right\|_{L^{2}(U)} \leq \epsilon K, \quad \forall \epsilon>0
$$

E por conseguinte

$$
\left\|\frac{\partial u^{\epsilon}}{\partial y}\right\|_{L^{2}(U)} \rightarrow 0, \quad \epsilon \rightarrow 0
$$

\subsection{Colocação abstrata e a existência de soluções}

Procedendo do modo análogo como em [17, 9], estamos interessados em escrever os problemas (1.7) e (1.5) numa forma abstrata e estudar a existência de soluções aplicando o Teorema de Schauder sobre o ponto fixo

\subsubsection{Colocação abstrata e existência de soluções do problema (1.7)}

Definimos a forma bilinear contínua $a_{\epsilon}: H^{1}(\Omega) \times H^{1}(\Omega) \rightarrow \mathbb{R}$, com $\epsilon \geq 0$ dado por

$$
a_{\epsilon}(u, v)=\int_{\Omega} \frac{\partial u}{\partial x} \frac{\partial v}{\partial x} d y d x+\frac{1}{\epsilon^{2}} \int_{\Omega} \frac{\partial u}{\partial y} \frac{\partial v}{\partial y} d y d x+\int_{\Omega} u v d y d x, \text { para } \epsilon>0
$$


onde $u, v \in H^{1}(\Omega)$.

Proposição 2.2.1 A forma bilinear (2.6) satifaz as seguintes propriedades:

i) $a_{\epsilon}$ é simétrica, isto é, $a_{\epsilon}(u, v)=a_{\epsilon}(v, u) \quad \forall u, v \in H^{1}(\Omega)$;

ii) $a_{\epsilon}$ é positiva, isto é, $a_{\epsilon}(u, u)>0 \quad \forall u \in H^{1}(\Omega)$;

iii) $a_{\epsilon}$ é coerciva em $H^{1}(\Omega)$, isto é, existe $\alpha>0$ tal que para todo $\epsilon>0$ tal que $a_{\epsilon}(u, u) \geq \alpha\|u\|_{H^{1}(\Omega)^{\prime}}^{2}$;

iv) $a_{\epsilon}$ contínua em $H^{1}(\Omega)$, isto é, existe uma constante positiva $C$ tal que $\left|a_{\epsilon}(u, v)\right| \leq C|u|_{H^{1}(\Omega)}|v|_{H^{1}(\Omega)}$.

Demonstração. Os ítens i) e ii) seguem imediatamente da definição de $a_{\epsilon}$. Para provar o ítem iii) vamos dividir em dois casos:

a) Para $\epsilon \in(0,1)$ temos que para todo $u \in H^{1}(\Omega)$

$$
a_{\epsilon}(u, u)=\|u\|_{H_{\epsilon}^{1}(\Omega)}^{2} \geq \alpha\|u\|_{H^{1}(\Omega)^{\prime}}^{2}
$$

pois, $\|\cdot\|_{H^{1}(\Omega)} \leq\|\cdot\|_{H_{\epsilon}^{1}(\Omega)}$ para todo $\epsilon \in(0,1)$. Observe que para $\epsilon \in(0,1)$ a forma bilinear $a_{\epsilon}$ é uniformemente coerciva pois, $\alpha=1$ é independente de $\epsilon$.

b) Para $\epsilon>1$ temos que para todo $u \in H^{1}(\Omega)$

$$
\begin{aligned}
a_{\epsilon}(u, u) & =\int_{\Omega}\left\{\left(\frac{\partial u}{\partial x}\right)^{2}+\frac{1}{\epsilon^{2}}\left(\frac{\partial u}{\partial y}\right)^{2}+u^{2}\right\} d y d x=\frac{1}{\epsilon^{2}} \int_{\Omega}\left\{\epsilon^{2}\left(\frac{\partial u}{\partial x}\right)^{2}+\left(\frac{\partial u}{\partial y}\right)^{2}+\epsilon^{2} u^{2}\right\} d y d x \\
& \geq \frac{1}{\epsilon^{2}} \int_{\Omega}\left\{\left(\frac{\partial u}{\partial x}\right)^{2}+\left(\frac{\partial u}{\partial y}\right)^{2}+u^{2}\right\} d y d x=\frac{1}{\epsilon^{2}}\|u\|_{H^{1}(\Omega)}^{2}=\alpha\|u\|_{H^{1}(\Omega)^{\prime}}^{2}
\end{aligned}
$$

onde $\alpha:=\alpha(\epsilon)=\epsilon^{-2}$, para $\epsilon>1$.

Finalmente tomando $u, v \in H^{1}(\Omega)$, usando a Desigualdade de Hölder e a definição do espaço $H^{1}(\Omega)$ temos

$$
\begin{aligned}
\left|a_{\epsilon}(u, v)\right| & \leq \int_{\Omega}\left|\frac{\partial u}{\partial x}\right|\left|\frac{\partial v}{\partial x}\right|+\frac{1}{\epsilon^{2}} \int_{\Omega}\left|\frac{\partial u}{\partial y}\right|\left|\frac{\partial v}{\partial y}\right| d y d x+\int_{\Omega}|u \| v| d y d x \\
& \leq\left\|\frac{\partial u}{\partial x}\right\|_{L^{2}(\Omega)}\left\|\frac{\partial v}{\partial x}\right\|_{L^{2}(\Omega)}+\frac{1}{\epsilon^{2}}\left\|\frac{\partial u}{\partial y}\right\|_{L^{2}(\Omega)}\left\|\frac{\partial v}{\partial y}\right\|_{L^{2}(\Omega)}+\|u\|_{L^{2}(\Omega)}\|v\|_{L^{2}(\Omega)} \\
& \leq\|u\|_{H^{1}(\Omega)}\|v\|_{H^{1}(\Omega)}+\frac{1}{\epsilon^{2}}\|u\|_{H^{1}(\Omega)}\|v\|_{H^{1}(\Omega)}+\|u\|_{H^{1}(\Omega)}\|v\|_{H^{1}(\Omega)} \\
& =\left(2+\frac{1}{\epsilon^{2}}\right)\|u\|_{H^{1}(\Omega)}\|v\|_{H^{1}(\Omega)}=C\|u\|_{H^{1}(\Omega)}\|v\|_{H^{1}(\Omega)}
\end{aligned}
$$

onde $C:=C(\epsilon)=2+\epsilon^{-2}$, onde $\epsilon>0$. Ficando assim provado o ítem iv).

Denotemos por $\langle\cdot, \cdot\rangle$ o produto interno em $L^{2}(\Omega)$. Temos 
Proposição 2.2.2 Dado $h \in L^{2}(\Omega)$ existe único $u \in H^{1}(\Omega)$ tal que

$$
a_{\epsilon}(u, v)=\langle h, v\rangle \quad v \in H^{1}(\Omega)
$$

Demonstração. Segue do Teorema de Lax-Milgram (Teorema C.2.2), devido a Proposição 2.2.1.

Corolário 2.2.3 Se $u \in H^{1}(\Omega)$ é tal que $a_{\epsilon}(u, v)=\langle h, v\rangle$, para todo $v \in H^{1}(\Omega)$ e algum $h \in L^{2}(\Omega)$, então $u \in H^{2}(\Omega)$ e satisfaz

$$
\left\{\begin{array}{c}
-\frac{\partial^{2} u}{\partial x^{2}}-\frac{1}{\epsilon^{2}} \frac{\partial^{2} u}{\partial y^{2}}+u=h, \quad \text { em } \Omega \\
\frac{\partial u}{\partial x} N_{1}+\frac{1}{\epsilon^{2}} \frac{\partial u}{\partial y} N_{2}=0 \quad \text { qs. na } \partial \Omega,
\end{array}\right.
$$

Prova. Segue da Proposição 2.2.2 que $u \in H^{1}(\Omega)$ é único. Agora usando a Proposição 2.2.1 e como $\Omega$ é um domínio Lipschitz contínua então pelo Teorema de Grisvard sobre a regularidade das soluções fracas de um problema de Neumann temos que $u \in H^{2}(\Omega)$, logo podemos aplicar o Teorema de Green em (2.6)

$$
a_{\epsilon}(u, v)=\int_{\Omega}\left\{-\frac{\partial^{2} u}{\partial x^{2}}-\frac{1}{\epsilon^{2}} \frac{\partial^{2} u}{\partial y^{2}}+u\right\} v d y d x+\int_{\partial \Omega} v\left\{\frac{\partial u}{\partial x} N_{1}+\frac{1}{\epsilon^{2}} \frac{\partial u}{\partial y} N_{2}\right\} d y d x,
$$

para $u \in H^{2}(\Omega)$ e $\forall v \in H^{1}(\Omega)$. Donde escolhendo $v$ convenientemente vemos $u$ satisfaz (2.8) como queriamos.

Como consequência, usando a Proposição 2.2.2 e o Corolário 2.2.3 definimos o operador linear $\mathcal{A}_{\epsilon}: D\left(\mathcal{A}_{\epsilon}\right) \subset L^{2}(\Omega) \rightarrow L^{2}(\Omega)$ associado a forma bilinear $a_{\epsilon}$ por

$$
\begin{gathered}
\mathcal{A}_{\epsilon} u=-\frac{\partial^{2} u}{\partial x^{2}}-\frac{1}{\epsilon^{2}} \frac{\partial^{2} u}{\partial y^{2}}+u, \\
D\left(\mathcal{A}_{\epsilon}\right)=\left\{u \in H^{2}(\Omega): \frac{\partial u}{\partial x} N_{1}+\frac{1}{\epsilon^{2}} \frac{\partial u}{\partial y} N_{2}=0 \text { quase sempre na } \partial \Omega\right\} .
\end{gathered}
$$

Notemos que o domínio $D\left(\mathcal{A}_{\epsilon}\right)$ é definido da seguinte maneira

$$
\left\{\begin{array}{l}
D\left(\mathcal{A}_{\epsilon}\right)=\left\{u \in H^{1}(\Omega) \mid \text { existe } w \in L^{2}(\Omega) \text { tal que para todo } v \in H^{1}(\Omega)\right. \text { temos } \\
\left.a_{\epsilon}(u, v)=\langle w, v\rangle\right\} \\
\mathcal{A}_{\epsilon} u:=w, \quad u \in D\left(\mathcal{A}_{\epsilon}\right) .
\end{array}\right.
$$

Agora considerando um espaço de Hilbert abstrato $\mathcal{H}$ munido de um produto interno $\langle\cdot, \cdot\rangle_{\mathcal{H}}$ sobre um corpo $\mathbb{K}(=\mathbb{R}$ ou $\mathbb{C})$ e um operador linear $T: D(T) \subset \mathcal{H} \rightarrow \mathcal{H}$ temos as seguintes definições

i) $T: D(T) \subset \mathcal{H} \rightarrow \mathcal{H}$ éfechado se $x_{n} \rightarrow x \quad$ e $\quad T x_{n} \rightarrow y \quad \Rightarrow \quad x \in D(T)$ e $\quad T x=y$;

ii) $T: D(T) \subset \mathcal{H} \rightarrow \mathcal{H}$ é densamente definido se $D(T)$ é denso em $\mathcal{H}$, isto é, $\overline{D(T)}=\mathcal{H}$; 
iii) O gráfico do operador $T$ em $\mathcal{H} \times \mathcal{H}$ é o conjunto definido por

$$
\operatorname{Graf}(T)=\{(x, T x): x \in D(T)\} .
$$

Proposição 2.2.4 O operador Té fechado se e somente se Graf(T) é fechado.

Demonstração. $\quad(\Rightarrow)$ Suponhamos que $T$ é fechado. Seja $(x, y) \in \overline{\operatorname{Graf}(T)}$ por definição existe $\left(x_{n}, T x_{n}\right) \in \operatorname{Graf}(T)$ tais que $\left(x_{n}, T x_{n}\right) \rightarrow(x, y)$ isto é, $x_{n} \rightarrow x$ e $T x_{n} \rightarrow y$. Como $T$ é fechado então $x \in D(T)$ e $T x=y$ o que implica que $(x, y) \in \operatorname{Graf}(T)$.

$(\Leftarrow)$ Suponhamos que $\operatorname{Graf}(T)$ é fechado. Se tomarmos a sequência $x_{n} \in D(T)$ tal que $x_{n} \rightarrow x$ e $T x_{n} \rightarrow y$ então $\left(x_{n}, T x_{n}\right) \rightarrow(x, y)$ e como $\operatorname{Graf}(T)$ é fechado temos que $(x, y) \in \operatorname{Graf}(T)$ o que implica que $x \in D(T)$ e $T x=y$ mostrando assim que $T$ é fechado.

Como o espaço de Hilbert $\mathcal{H}$ pode ser identificado com o seu dual definimos $T^{*}: D\left(T^{*}\right) \subset \mathcal{H} \rightarrow \mathcal{H}$ o operador adjunto de $T$ do seguinte modo

$$
D\left(T^{*}\right)=\left\{u \in \mathcal{H}: v \mapsto\langle T v, u\rangle_{\mathcal{H}}: D(T) \rightarrow \mathbb{K} \text { é limitado }\right\}
$$

Se $u \in D\left(T^{*}\right), T^{*} u$ é único elemento de $\mathcal{H}$ tal que

$$
\left\langle v, T^{*} u\right\rangle_{\mathcal{H}}=\langle T v, u\rangle_{\mathcal{H}} \forall v \in D(T)
$$

Definição 2.2.5 O operador $T: D(T) \subset \mathcal{H} \rightarrow \mathcal{H}$ densamente definido é dito simétrico se e somente se $\langle T u, v\rangle=\langle u, T v\rangle \quad \forall u, v \in D(T)$. Equivalentemente $T$ é dito simétrico se é densamente definido e $T \subseteq T^{*}$, isto equivale a dizer que $T^{*}$ é uma extensão de $T$. E diremos que $T$ é auto-adjunto se $T=T^{*}$.

Proposição 2.2.6 Se Té um operador fechado, então $T^{*}$ é um operador fechado.

Demonstração. Pela Proposição 2.2.4 temos que $T$ é fechado ou seja, $x_{n} \in D(T)$ e $x \in \mathcal{H}$ é tal que $x_{n} \rightarrow x$ e $T x_{n} \rightarrow y$ implica que $x \in D(T)$ e $T x=y$. Pela Definição 2.2.5 temos que $\overline{D(T)}=\mathcal{H}$ e $T \subseteq T^{*}$ o que implica que $D(T) \subseteq D\left(T^{*}\right)$ e portanto $x_{n} \in D\left(T^{*}\right)$ e $T^{*} x_{n} \rightarrow y$ e consequentemente $x \in D\left(T^{*}\right)$ e $T^{*} x=y$.

Proposição 2.2.7 Se $T: D(T) \subset \mathcal{H} \rightarrow \mathcal{H}$ é densamente definido, simétrico e sobrejetor, então T é autoadjunto isto é, $D(T)=D\left(T^{*}\right) e\langle v, T u\rangle_{\mathcal{H}}=\langle T v, u\rangle_{\mathcal{H}} \forall u, v \in D(T)$.

Demonstração. Como $T$ é tal que $\overline{D(T)}=\mathcal{H}$ e simétrico implica que $T \subseteq T^{*}$. Com efeito, dado $u, v \in D(T)$ então $v \in D\left(T^{*}\right)$ e $\langle T u, v\rangle_{\mathcal{H}}=\left\langle u, T^{*} v\right\rangle_{\mathcal{H}}=\langle u, T v\rangle_{\mathcal{H}}$. Para provar que $D\left(T^{*}\right) \supseteq D(T)$ é necessário usar a sobrejetividade. Para cada $v \in D(T)$ temos $\langle T u, v\rangle_{\mathcal{H}}=\langle u, T v\rangle_{\mathcal{H}}, u \in D(T)$. Logo $v \in D\left(T^{*}\right)$ e $T^{*} v=T v \Rightarrow T^{*}=T$ e $D\left(T^{*}\right) \supseteq D(T)$. Agora usando de novo o fato de que $T$ é sobrejetor, temos que para cada $w \in D\left(T^{*}\right)$ existe único $u \in D(T)$ tal que $\left\langle v, T^{*} w\right\rangle_{\mathcal{H}}=\langle v, T u\rangle_{\mathcal{H}} \quad \forall v \in \mathcal{H}$. Assim 
$0=\left\langle v, T^{*} w-T u\right\rangle_{\mathcal{H}} \Rightarrow T^{*} w=T u \Rightarrow w=u, \log \mathrm{w} w \in D(T)$. Portanto $T^{*}=T$ e $D(T) \supseteq D\left(T^{*}\right)$. E assim concluimos que $D(T)=D\left(T^{*}\right)$ e $\langle v, T u\rangle_{\mathcal{H}}=\langle T v, u\rangle_{\mathcal{H}}$.

Com base no que fizemos acima tomando $\mathcal{H}=L^{2}(\Omega)$ e $T=\mathcal{A}_{\epsilon}$ podemos concluir que $\mathcal{A}_{\epsilon}$ é um operador fechado, densamente definido, simétrico e auto-adjunto

Proposição 2.2.8 Seja $\mathcal{A}_{\epsilon}: D\left(\mathcal{A}_{\epsilon}\right) \subset L^{2}(\Omega) \rightarrow L^{2}(\Omega)$

i) $\mathcal{A}_{\epsilon}$ é bijetora e possui inversa contínua;

ii) $\mathcal{A}_{\epsilon}^{-1}$ é compacto.

Demonstração. i) Segue da Proposição 2.2.2 e pela definição de $D\left(\mathcal{A}_{\epsilon}\right)$ que existe único $u \in H^{2}(\Omega)$ tal que $\mathcal{A}_{\epsilon} u=w \operatorname{com} w \in L^{2}(\Omega)$. O que implica que a imagem de $\mathcal{A}_{\epsilon}$ é $L^{2}(\Omega)$ isto é, $\mathscr{R}\left(\mathcal{A}_{\epsilon}\right)=L^{2}(\Omega)$ o que implica que $\mathcal{A}_{\epsilon}$ é sobrejetora. Para mostrar que $\mathcal{A}_{\epsilon}$ é injetora usamos a Proposição 2.2.2 e o Corolário 2.2.3. Existe único $u \in H^{2}(\Omega)$ tal que $\mathcal{A}_{\epsilon} u=h$, então se $h=0$ implica que $u=0$, o que mostra que $\mathcal{A}_{\epsilon}$ é injetora e portanto bijetora e a inversa é definida por

$$
\mathcal{A}_{\epsilon}^{-1}: L^{2}(\Omega) \rightarrow L^{2}(\Omega)
$$

Segue da Proposição 2.2.4 que o operador $\mathcal{A}_{\epsilon}$ é fechado e por [36] temos que se $\mathcal{A}_{\epsilon}$ é inversível então $\operatorname{Graf}\left(\mathcal{A}_{\epsilon}\right)=\operatorname{Graf}\left(\mathcal{A}_{\epsilon}^{-1}\right)$ e consequentemente $\mathcal{A}_{\epsilon}^{-1}$ é fechado e portanto, pelo Teorema de Gráfico Fechado [13, Teorema 2.9] temos que

$$
\mathcal{A}_{\epsilon}^{-1}: L^{2}(\Omega) \rightarrow L^{2}(\Omega) \quad \text { é um operador linear e contínuo. }
$$

ii) Pelo ítem anterior temos que $\mathcal{A}_{\epsilon}^{-1}$ é um operador linear e contínuo. Como $\mathscr{R}\left(\mathcal{A}_{\epsilon}^{-1}\right) \subset H^{2}(\Omega) \subset L^{2}(\Omega)$ e como $H^{2}(\Omega)$ está imerso compactamente em $L^{2}(\Omega)$, concluimos que $\mathcal{A}_{\epsilon}^{-1}$ é compacto conforme queriamos mostrar.

Agora usando o fato de que para $H^{-s}(\Omega)$ é o dual de $H^{s}(\Omega)$ para $s>0$ e em particular para $s=1$ temos

$$
H^{1}(\Omega) \subset L^{2}(\Omega) \subset H^{-1}(\Omega),
$$

portanto $\mathcal{A}_{\epsilon}$ pode ser visto como um operador que age do espaço $H^{1}(\Omega)$ para o seu dual $H^{-1}(\Omega)$ tal que para todo $u \in H^{1}(\Omega)$

$$
\left\langle\mathcal{A}_{\epsilon} u, v\right\rangle_{H^{-1}(\Omega), H^{1}(\Omega)}=\int_{\Omega}\left\{\frac{\partial u}{\partial x} \frac{\partial v}{\partial x}+\frac{1}{\epsilon^{2}} \frac{\partial u}{\partial y} \frac{\partial v}{\partial y}+u v\right\} d y d x, \quad \text { para } \quad v \in H^{1}(\Omega) .
$$


Assim, o problema (1.7) pode ser escrito como $\mathcal{A}_{\epsilon} u=F_{\epsilon}(u), \epsilon>0 \operatorname{com} F_{\epsilon}: H^{1}(\Omega) \rightarrow H^{-s}(\Omega)$ para $1 / 2<s<1$, onde

$$
\begin{gathered}
F_{\epsilon}=\widehat{F}+\widehat{F_{\epsilon}} \\
\langle\widehat{F}(u), v\rangle=\int_{\Omega} f(u) v d \xi, \quad\left\langle\widehat{F}_{\epsilon}(u), v\right\rangle=\frac{1}{\epsilon^{\alpha}} \int_{o_{\epsilon}} g(u) v d \xi,
\end{gathered}
$$

para $u \in H^{1}(\Omega)$ e $v \in H^{1}(\Omega)$. Assim se $F_{\epsilon} \in H^{-s}(\Omega)$ a solução de $\mathcal{A}_{\epsilon} u=F_{\epsilon}(u)$ é a solução fraca do problema (1.7). Portanto temos que $u^{\epsilon}$ é uma solução do problema (1.7), se e somente se $u^{\epsilon} \in H^{1}(\Omega)$ satisfaz $u^{\epsilon}=\mathcal{A}_{\epsilon}^{-1} \circ F_{\epsilon}\left(u^{\epsilon}\right)$. Assim, $u^{\epsilon}$ deve ser o ponto fixo da aplicação não linear

$$
\mathcal{A}_{\epsilon}^{-1} \circ F_{\epsilon}: H^{1}(\Omega) \rightarrow H^{1}(\Omega)
$$

Lema 2.2.9 Se a sequência $u^{\epsilon} \in H^{1}(\Omega)$ é tal que $\left\|u^{\epsilon}\right\|_{H^{1}(\Omega)} \leq M$, para alguma constante positiva, então a família $\left\{\mathcal{A}_{\epsilon}^{-1} \circ F_{\epsilon}\left(u^{\epsilon}\right)\right\}_{\epsilon>0}$ é compacta.

Prova. Temos que a sequência $u^{\epsilon} \in H^{1}(\Omega)$ é limitada, isto é, existe $M>0$ tal que $\|u\|_{H^{1}(\Omega)} \leq M$. Então pelo Teorema A.1.11 podemos extrair uma subsequência que ainda denotaremos por $u^{\epsilon}$ e uma função $u \in H^{1}(\Omega)$ tal que

$$
u^{\epsilon} \rightarrow u, w-H^{1}(\Omega)
$$

Agora consideremos $w^{\epsilon}=\mathcal{A}_{\epsilon}^{-1} \circ F_{\epsilon}\left(u^{\epsilon}\right)$ que equivale a $\mathcal{A}_{\epsilon} w^{\epsilon}=F_{\epsilon}\left(u^{\epsilon}\right)$ com $\left\|u^{\epsilon}\right\|_{H^{1}(\Omega)} \leq M$. Para mostrar que a família $\left\{\mathcal{A}_{\epsilon}^{-1} \circ F_{\epsilon}\left(u^{\epsilon}\right)\right\}_{\epsilon>0}$ é suficiente mostrar que $\left\|w^{\epsilon}\right\|_{H^{1}(\Omega)} \leq K$, para alguma constante positiva. Usando fato de que $f$ e $g$ são funções limitadas e $H^{1}(\Omega) \hookrightarrow L^{2}(\Omega)$ ser uma imersção compacta temos

$$
\begin{aligned}
\left\|w^{\epsilon}\right\|_{H_{\epsilon}^{1}(\Omega)}^{2} & =\int_{\Omega}\left\{\left|\frac{\partial^{2} w^{\epsilon}}{\partial x^{2}}\right|^{2}+\frac{1}{\epsilon^{2}}\left|\frac{\partial^{2} w^{\epsilon}}{\partial y^{2}}\right|^{2}+\left|w^{\epsilon}\right|^{2}\right\} d y d x=\int_{\Omega} F_{\epsilon}\left(u^{\epsilon}\right) w^{\epsilon} d y d x \\
& \leq \int_{\Omega}\left|F_{\epsilon}\left(u^{\epsilon}\right)\left\|w^{\epsilon} \mid d y d x \leq\right\| F_{\epsilon}\left(u^{\epsilon}\right)\left\|_{L^{2}(\Omega)}\right\| w^{\epsilon}\left\|_{L^{2}(\Omega)} \leq K\right\| w^{\epsilon} \|_{H^{1}(\Omega)} .\right.
\end{aligned}
$$

Por outro lado para $\epsilon \in(0,1)$ temos $\|\cdot\|_{H^{1}(\Omega)} \leq\|\cdot\|_{H_{\epsilon}^{1}(\Omega)}$. Assim

$$
\left\|w^{\epsilon}\right\|_{H^{1}(\Omega)}^{2} \leq\left\|w^{\epsilon}\right\|_{H_{\epsilon}^{1}(\Omega)}^{2} \leq K\left\|w^{\epsilon}\right\|_{H^{1}(\Omega)} \quad \Rightarrow \quad\left\|w^{\epsilon}\right\|_{H^{1}(\Omega)} \leq K
$$

Assim concluimos que a família $\left\{\mathcal{A}_{\epsilon}^{-1} \circ F_{\epsilon}\left(u^{\epsilon}\right)\right\}_{\epsilon>0}$ é limitada e pelo Teorema A.1.11 portanto possui uma subsequência convergente. Logo a família de operadores $\mathcal{A l}_{\epsilon}^{-1} \circ F_{\epsilon}: H^{1}(\Omega) \rightarrow H^{1}(\Omega)$ é compacta com $\left\|u^{\epsilon}\right\|_{H^{1}(\Omega)} \leq M$.

Consequentemente, a existência de soluções do problema (1.7) segue do Teorema de Schauder sobre o ponto fixo (Teorema D.2.20). 


\subsubsection{Colocação abstrata e existência de soluções do problema (1.5)}

Da prova da propriedade iii) da Proposição 2.1.1 temos que $a_{\epsilon}(\cdot, \cdot)$ define a norma em $H_{\epsilon}^{1}(\Omega)$ dada por $\|u\|_{H_{\epsilon}^{1}(\Omega)}=\left(a_{\epsilon}(u, u)\right)^{1 / 2}$. Facilmente vemos que $\|u\|_{H_{\epsilon}^{1}(\Omega)} \rightarrow \infty$, quando $\epsilon \rightarrow 0$ se $\frac{\partial u}{\partial y}(x, y) \neq 0$ em $L^{2}(\Omega)$. Com efeito podemos introduzir para $u \in H^{1}(\Omega)$ o seguinte

$$
\lim _{\epsilon \rightarrow 0} a_{\epsilon}(u, u)=\left\{\begin{array}{cl}
p(x)\left\{\left\|\frac{\partial u}{\partial x}\right\|_{L^{2}(0,1)}^{2}+\|u\|_{L^{2}(0,1)}^{2}\right\}, & \text { se } \frac{\partial u}{\partial y}(x, y)=0 \\
\infty & \text { caso contrário. }
\end{array}\right.
$$

Nesse sentido a família $a_{\epsilon}$, com $\epsilon>0$ tem limite finito se e somente se $u \in \widehat{H}^{1}(0,1)$ onde $\widehat{H}^{1}(0,1)$ denota o espaço

$$
\widehat{H}^{1}(0,1)=\left\{u \in H^{1}(\Omega): \frac{\partial u}{\partial y}(x, y)=0\right\}
$$

É claro que $\widehat{H}^{1}(0,1)$ é um espaço de Hilbert com a norma

$$
\|u\|_{\widehat{H}^{1}(0,1)}^{2}=p(x)\left\{\left\|\frac{\partial u}{\partial x}\right\|_{L^{2}(0,1)}^{2}+\|u\|_{L^{2}(0,1)}^{2}\right\}
$$

definido pelo produto interno

$$
(\phi, \varphi)_{\widehat{H}^{1}(0,1)}=\int_{0}^{1} p(x)\left\{\frac{\partial \phi}{\partial x} \frac{\partial \varphi}{\partial x}+\phi \varphi\right\} d x
$$

Além disso temos que $H^{1}(0,1)$ e $\widehat{H}^{1}(0,1)$ possuem normas equivalentes, pois se $u \in H^{1}(\Omega)$ com $\frac{\partial u}{\partial y}(x, y)=0$ existe duas constantes positivas $C 1$ e $C_{2}$ tais que

$$
C_{1}\|\cdot\|_{H^{1}(0,1)} \leq\|\cdot\|_{\widehat{H}^{1}(0,1)} \leq C_{2}\|\cdot\|_{H^{1}(0,1)}
$$

Com efeito, pelas nossas suposições temos que $0<G_{0} \leq G(x) \leq G_{1}$ e $0<b_{0} \leq b(x) \leq b_{1}$ o que implica que $0<b_{0}+G_{0} \leq b(x)+G(x) \leq b_{1}+G_{1} \stackrel{\text { def }}{=} H_{1}$. Assim

$$
\min _{x \in(0,1)}\{p(x)\} \int_{0}^{1}\left\{u_{x}^{2}+u^{2}\right\} d x \leq \int_{0}^{1} p(x)\left\{u_{x}^{2}+u^{2}\right\} d x \leq \max _{x \in(0,1)}\{p(x)\} \int_{0}^{1}\left\{u_{x}^{2}+u^{2}\right\} d x,
$$

ou seja, $C_{1}\|u\|_{H^{1}(0,1)} \leq\|u\|_{\widehat{H}^{1}(0,1)} \leq C_{2}\|u\|_{H^{1}(0,1)}$ com as constantes positvas definidas respetivamente por $C_{1}=\sqrt{b_{0}+G_{0}}$ e $C_{2}=\sqrt{b_{1}+G_{1}}$.

Assim similarmente como no caso anterior (formulação abstrata do problema (1.7)) para escrevermos a forma abstrata do problema (1.5) definimos a forma bilinear $a_{0}: \widehat{H}^{1}(0,1) \times \widehat{H}^{1}(0,1) \rightarrow \mathbb{R}$ pela seguinte fórmula

$$
a_{0}(u, v)=\int_{0}^{1} p(x)\left\{u_{x} v_{x}+u v\right\} d x
$$

onde $u, v \in \widehat{H}^{1}(0,1)$ e $p:(0,1) \rightarrow(0, \infty)$ é uma função positiva definida em (1.6). 
Observação 2.2.10 A forma bilinear $a_{0}$ satisfaz as propriedades da Proposição 2.1.1, istoé, simétrica, positiva, coerciva e contínua.

Denotando por $\langle\cdot, \cdot\rangle$ o produto interno em $\hat{L}^{2}(0,1)$ onde $\hat{L}^{2}(0,1)$ é o espaço $L^{2}(0,1)$ com o produto interno conveniente dado por

$$
\langle u, v\rangle=\int_{0}^{1} p(x) u(x) v(x) d x
$$

Proposição 2.2.11 Dado $h \in \hat{L}^{2}(0,1)$ existe único $u \in \widehat{H}^{1}(0,1)$ tal que

$$
a_{0}(u, v)=\langle h, v\rangle, \quad v \in \widehat{H}^{1}(0,1)
$$

Demonstração. Segue do Teorema de Lax-Milgram devido, a Observação 2.2.10.

Corolário 2.2.12 Se $u \in \widehat{H}^{1}(0,1)$ é tal que $a_{0}(u, v)=\langle h, v\rangle$ para todo $v \in \widehat{H}^{1}(0,1)$ e para algum $h \in \hat{L}^{2}(0,1)$, então $u \in \widehat{H}^{2}(0,1)$ e satisfaz

$$
\left\{\begin{array}{c}
-\frac{1}{p(x)}\left(p(x) u_{x}\right)_{x}+u=h, \quad \text { em } \quad(0,1) \\
u_{x}(0)=u_{x}(1)=0 .
\end{array}\right.
$$

Prova. A Proposição 2.2.11 garante que $u \in \widehat{H}^{1}(0,1)$ é único. Agora pela Observação 2.2.10 e pela convexidade do intervalo $(0,1)$, temos pelo Teorema de Grisvard sobre a regularidade de um problema de Neumann que $u \in \widehat{H}^{2}(0,1)$. Logo podemos aplicar o Teorema de Green em (2.13) obtendo,

$$
a_{0}(u, v)=\int_{0}^{1}\left\{-\frac{1}{p(x)}\left(p(x) u_{x}(x)\right)_{x}+u(x)\right\} v(x) p(x) d x+u_{x}(1) p(1) v(1)-u_{x}(0) p(0) v(0),
$$

donde escolhendo $v$ convenientemente e pela arbitrariedade de $v(1), v(0)$ e $p(0), p(1)$ são positivos temos que $u \in \widehat{H}^{1}(0,1)$ satisfaz o problema clássico (2.15).

Com base na Proposição 2.2.11 e o Corolário 2.2.12 definimos $\mathcal{A}_{0}: D(\mathcal{A}): \hat{L}^{2}(0,1) \rightarrow \hat{L}^{2}(0,1)$ o operador linear associado a forma bilinear $a_{0}$ dada por

$$
\begin{gathered}
\mathcal{A}_{0} u=-\frac{1}{p(x)}\left(p(x) u_{x}(x)\right)_{x}+u(x) \\
D\left(\mathcal{A}_{0}\right)=\left\{u \in H^{2}(0,1): u_{x}(1)=u_{x}(0)=0\right\} .
\end{gathered}
$$

O domínio $D\left(\mathcal{A}_{0}\right)$ é definido por

$$
\left\{\begin{array}{l}
D\left(\mathcal{A}_{0}\right)=\left\{u \in H^{1}(0,1), v \mapsto a_{0}(u, v) \text { é contínua em } H^{1}(0,1)\right\} \\
a_{0}(u, v)=\left\langle\mathcal{A}_{0} u, v\right\rangle, \text { que define } \mathcal{A}_{0} u \in \hat{L}^{2}(0,1) .
\end{array}\right.
$$


Observação 2.2.13 O operador $\mathcal{A}_{0}$ é densamente definido, fechado, simétrico, auto-adjunto, com inversa contínua e compacto.

Agora usando o fato de que para $H^{-s}(0,1)$ é o dual de $H^{s}(0,1)$ para $s>0$ e em particular para $s=1$ temos

$$
H^{1}(0,1) \subset \hat{L}^{2}(0,1) \subset H^{-1}(0,1),
$$

portanto $\mathcal{A}_{0}$ pode ser visto como um operador que age do espaço $H^{1}(0,1)$ para o seu dual $H^{-1}(0,1)$ tal que para todo $u \in H^{1}(0,1)$ tem-se

$$
\left\langle\mathcal{A}_{0} u, v\right\rangle_{H^{-1}(0,1), H^{1}(0,1)}=\int_{0}^{1}\left\{u_{x}(x) v_{x}(x)+u(x) v(x)\right\} p(x) d x, \quad v \in H^{1}(0,1) .
$$

Assim o problema (1.5) pode ser escrito como $\mathcal{A}_{0} u=F(u)$, onde $F_{0}: H^{1}(0,1) \rightarrow H^{-s}(0,1)$ com $1 / 2<s<1$ é definido por

$$
\left\langle F_{0}(u), v\right\rangle=\int_{0}^{1}\left\{f(u(x))+\frac{\mu(x)}{p(x)} g(u(x))\right\} v(x) p(x) d x, \quad v \in H^{s}(0,1),
$$

onde $\mu:(0,1) \rightarrow(0, \infty)$ é uma função suave definida em (1.6). Assim se $F_{0} \in H^{-s}(0,1)$ para $1 / 2<s<1$ a solução de $\mathcal{A}_{0} u=F_{0}(u)$ é a solução fraca do problema (1.5). Portanto $u$ é a solução do problema (1.5) se e somente se $u \in H^{1}(0,1)$ satisfaz $u=\mathcal{A}_{0}^{-1} \circ F_{0}(u)$. Logo $u$ deve ser o ponto fixo da aplicação não-linear

$$
\mathcal{A}_{0}^{-1} \circ F_{0}: H^{1}(0,1) \rightarrow H^{1}(0,1)
$$

Lema 2.2.14 Se a sequência $u_{n} \in H^{1}(0,1)$ é limitada então a família $\left\{\mathcal{A}_{0}^{-1} \circ F_{0}\left(u_{n}\right)\right\}$ é compacta.

Prova. Temos que a sequência $u_{n}$ em $H^{1}(0,1)$ é limitada então existe uma constante positiva $M$ tal que $\left\|u_{n}\right\|_{H^{1}(0,1)} \leq M$. Logo pelo Teorema A.1.11 podemos extrair uma subsequência que ainda denotaremos por $u_{n}$ e uma funcção $u \in H^{1}(0,1)$ tal que

$$
u_{n} \rightarrow u \quad \text { em } w-H^{1}(0,1), \quad \text { quando } n \rightarrow \infty .
$$

Agora consideremos $\omega_{n}=\mathcal{A}_{0}^{-1} \circ F_{0}\left(u_{n}\right)$ que é equivalente a $\mathcal{A}_{0} \omega_{n}=F_{0}\left(u_{n}\right)$ sendo $\omega_{n}$ solução fraca. Para mostrar que $\left\{\mathcal{A}_{0}^{-1} \circ F_{0}\left(u_{n}\right)\right\}$ possui uma subsequência convergente é suficiente mostrar que $\omega_{n}$ é limitada em $H^{1}(0,1)$. Para tal usando o fato de que $H^{1}(0,1)$ está imerso compactamente em $\hat{L}^{2}(0,1)$ 
temos

$$
\begin{aligned}
\left\|\omega_{n}\right\|_{H^{1}(0,1)}^{2} & =\int_{0}^{1} p(x)\left\{\left|\omega_{x}^{n}\right|^{2}+\left|\omega_{n}\right|\right\} d x \leq \int_{0}^{1}\left\{p(x)\left|f\left(u_{n}\right)\right|+\mu(x)\left|g\left(u_{n}\right)\right|\right\}\left|\omega_{n}\right| d x \\
& \leq\left\{\int_{0}^{1}\left\{p(x)\left|f\left(u_{n}\right)\right|+\mu(x)\left|g\left(u_{n}\right)\right|\right\}^{2} d x\right\}^{1 / 2}\left\|\omega_{n}\right\|_{\hat{L}^{2}(0,1)} \\
& \leq\left\{2 \int_{0}^{1} p^{2}(x)\left|f\left(u_{n}\right)\right|^{2} d x+2 \int_{0}^{1} \mu^{2}(x)\left|g\left(u_{n}\right)\right|^{2} d x\right\}^{1 / 2}\left\|\omega_{n}\right\|_{\hat{L}^{2}(0,1)} \\
& \leq \sqrt{2}\left\{H_{1}\|f\|_{L^{\infty}(\mathbb{R})}+\|\mu\|_{L^{\infty}(0,1)}\|g\|_{L^{\infty}(\mathbb{R})}\right\}^{1 / 2}\left\|\omega_{n}\right\|_{H^{1}(0,1)} .
\end{aligned}
$$

Portanto existe uma constante $K:=K(f, g, p, \mu)$ tal que $\left\|\omega_{n}\right\|_{H^{1}(0,1)} \leq K$. O que implica que a família $\left\{\mathcal{A}_{0}^{-1} \circ F_{0}\left(u_{n}\right)\right\}$ é limitada, ou seja que possui uma subsequência convergente pelo Teorema A.1.11. Logo a família $\mathcal{A}_{0}^{-1} \circ F_{0}: H^{1}(0,1) \rightarrow H^{1}(0,1)$ é compacta com $\left\|u_{n}\right\|_{H^{1}(0,1)} \leq M$.

Assim vemos que as soluções do problema limite (1.5) podem ser expressas como ponto fixo da aplicação (2.17) e consequentemente a existência de soluções do problema (1.5) segue do Teorema de Schuader sobre o ponto fixo (Teorema D.2.20) como queriamos observar.

\subsection{Caracterização dos domínios de definição}

Consideremos funções $b$ e $G:(0,1) \rightarrow \mathbb{R}^{+}$e a família de funções $H_{\epsilon}:(0,1) \rightarrow \mathbb{R}^{+}$para algum $\epsilon \in\left(0, \epsilon_{0}\right)$, com $0<\epsilon_{0} \leq 1$ e assumimos as seguintes hipóteses em relação as funções $b, G$ e $H_{\epsilon}$ :

(H1) As funções $b$ e $G$ são suaves, positivas e uniformemente limitadas e existem as constantes fixadas positivas $b_{0}, b_{1}, G_{0}$ e $G_{1}$ tais que $0<b_{0} \leq b(x) \leq b_{1}$ e $0<G_{0} \leq G(x) \leq G_{1}$ para todo $x \in(0,1)$ e a $G$ possui derivada uniformemente limitada.

(H2) As funções $H_{\epsilon}(\cdot)$ são do tipo (1.3) onde a função $H$ definida abaixo

$$
\begin{aligned}
H:[0,1] \times \mathbb{R} & \rightarrow \mathbb{R} \\
(x, y) & \mapsto H(x, y)
\end{aligned}
$$

é contínua na variável $x$ e uniformemente contínua na segunda variável $y$, isto é, para todo $\eta>0$, existe um $\delta>0$ tal que $\left|H(x, y)-H\left(x^{\prime}, y\right)\right|<\eta$ sempre que $x, x^{\prime} \in[0,1],\left|x-x^{\prime}\right|<\delta$ além disso $H(x, y)$ é não negativa e $l(x)$ - periódica na segunda variável y para todo $x \in[0,1]$, isto é $H(x, y+l(x))=H(x, y)$ para todo $y$, onde a função período $l:[0,1] \rightarrow \mathbb{R}$ é uniformemente limitada e positiva com $0<l_{0} \leq l(x) \leq l_{1}$ para todo $x \in[0,1]$.

Notemos que em nossas suposições permitimos o caso sem oscilação que ocorre quando $\beta=0$ em (1.3). Assim temos $H_{\epsilon}(x)=H(x, x) \operatorname{com} H_{\epsilon}$ independente de $\epsilon$. Dessa forma a faixa estreita $\theta_{\epsilon}$ não possui comportamento oscilatório. 
O seguinte resultado que também será bastante útil ao longo do trabalho.

Lema 2.3.1 Se $H_{\epsilon}$ é definido como em (1.3), então

$$
H_{\epsilon}(\cdot) \rightarrow \mu(\cdot)=\frac{1}{l(\cdot)} \int_{0}^{l(\cdot)} H(\cdot, s) d s \quad w^{*}-L^{\infty}(0,1)
$$

Prova. Temos que mostrar que

$$
\int_{0}^{1}\left\{H_{\epsilon}(x)-\frac{1}{l(x)} \int_{0}^{l(x)} H(x, s) d s\right\} \varphi(x) d x \rightarrow 0, \quad \epsilon \rightarrow 0, \quad \forall \varphi \in L^{1}(0,1) .
$$

Como o conjunto das funções simples é denso em $L^{1}(0,1)$ e toda a função simples pode ser escrita como combinação linear de funções características (Definição A.2.8), é suficiente supor $\varphi=\chi_{(e, f)}$ a função característica do intervalo $0 \leq e<f \leq 1$, isto é,

$$
\varphi(x)=\left\{\begin{array}{lll}
1, & \text { se } x \in(e, f) \\
0, & \text { se } x \notin(e, f)
\end{array}\right.
$$

Assim temos que estimar a seguinte integral

$$
I_{e, f}=\int_{e}^{f}\left\{H_{\epsilon}(x)-\frac{1}{l(x)} \int_{0}^{l(x)} H(x, s) d s\right\} d x .
$$

Para um $\eta>0$ suficientemente pequeno existe $n$ que define uma partição do intervalo $(e, f) \mathcal{P}=$ $\left\{e=x_{0}, x_{1}, x_{2}, \ldots, x_{n}=f\right\} \operatorname{com} e=x_{0}<x_{1}<x_{2}<\ldots<x_{n}=f$. Fixemos $\hat{x}_{i} \in J_{i} \stackrel{\text { def }}{=}\left[x_{i-1}, x_{i}\right]$ com $i=1,2, \ldots, n$ tais que

$$
\max _{i} \sup _{x \in J_{i}, y \in \mathbb{R}}\left|H(x, y)-H\left(\hat{x}_{i}, y\right)\right|<\eta
$$

Adicionando e subtraindo termos correspondentes em $I_{e, f}$ vemos que $I_{e, f}=\sum_{i=1}^{5} I_{e, f}^{i}$ onde:

$$
\begin{gathered}
I_{e, f}^{1}=\sum_{i=1}^{n} \int_{J_{i}}\left\{H\left(x, x / \epsilon^{\alpha}\right)-H\left(\hat{x}_{i}, x / \epsilon^{\alpha}\right)\right\} d x \\
I_{e, f}^{2}=\sum_{i=1}^{n} \int_{J_{i}}\left\{H\left(\hat{x}_{i}, x / \epsilon^{\alpha}\right)-\frac{1}{l\left(\hat{x}_{i}\right)} \int_{0}^{l\left(\hat{x}_{i}\right)} H\left(\hat{x}_{i}, s\right) d s\right\} d x \\
I_{e, f}^{3}=\sum_{i=1}^{n} \int_{J_{i}}\left\{\frac{1}{l\left(\hat{x}_{i}\right)} \int_{0}^{l\left(\hat{x}_{i}\right)} H\left(\hat{x}_{i}, s\right) d s-\frac{1}{l\left(\hat{x}_{i}\right)} \int_{0}^{l\left(\hat{x}_{i}\right)} H(x, s) d s\right\} d x \\
I_{e, f}^{4}=\sum_{i=1}^{n} \int_{J_{i}}\left\{\frac{1}{l\left(\hat{x}_{i}\right)} \int_{0}^{l\left(\hat{x}_{i}\right)} H(x, s) d s-\frac{1}{l\left(\hat{x}_{i}\right)} \int_{0}^{l(x)} H(x, s) d s\right\} d x \\
I_{e, f}^{5}=\sum_{i=1}^{n} \int_{J_{i}}\left\{\frac{1}{l\left(\hat{x}_{i}\right)} \int_{0}^{l(x)} H(x, s) d s-\frac{1}{l(x)} \int_{0}^{l(x)} H(x, s) d s\right\} d x .
\end{gathered}
$$


Temos:

$$
\begin{aligned}
& \left|I_{e, f}^{1}\right| \leq \sum_{i=1}^{n} \int_{J_{i}}\left|H\left(x, x / \epsilon^{\alpha}\right)-H\left(\hat{x}_{i}, x / \epsilon^{\alpha}\right)\right| d x<\sum_{i=1}^{n} \int_{J_{i}} \eta d x=\eta(f-e) . \\
& \left|I_{e, f}^{3}\right| \leq \sum_{i=1}^{n} \int_{J_{i}} \frac{1}{l\left(\hat{x}_{i}\right)}\left\{\int_{0}^{l\left(\hat{x}_{i}\right)}\left|H\left(\hat{x}_{i}, s\right)-H(x, s)\right| d s\right\} d x<\sum_{i=1}^{n} \int_{J_{i}} \frac{1}{l\left(\hat{x}_{i}\right)}\left\{\int_{0}^{l\left(\hat{x}_{i}\right)} \eta d s\right\} d x=\eta(f-e) . \\
& \left|I_{e, f}^{4}\right|=\left|\sum_{i=1}^{n} \int_{J_{i}}\left\{\frac{1}{l\left(\hat{x}_{i}\right)} \int_{0}^{l\left(\hat{x}_{i}\right)} H(x, s) d s-\frac{1}{l\left(\hat{x}_{i}\right)} \int_{0}^{l(x)} H(x, s) d s\right\} d x\right| \\
& \leq\left|\sum_{i=1}^{n} \int_{J_{i}}\left\{\frac{1}{l\left(\hat{x}_{i}\right)} \int_{0}^{l\left(\hat{x}_{i}\right)} H_{0} d s-\frac{1}{l\left(\hat{x}_{i}\right)} \int_{0}^{l(x)} H_{0} d s\right\} d x\right|=\left|\sum_{i=1}^{n} \int_{J_{i}} \frac{1}{l\left(\hat{x}_{i}\right)} \int_{l(x)}^{l\left(\hat{x}_{i}\right)} H_{0} d s d x\right| \\
& \leq \sum_{i=1}^{n} \int_{J_{i}} \frac{\left|l\left(\hat{x}_{i}\right)-l(x)\right|}{\left|l\left(\hat{x}_{i}\right)\right|} H_{0} d x \leq \frac{H_{0}}{l_{0}}\|\hat{l} \eta-l\|_{L^{\infty}(0,1)} \sum_{i=1}^{n} \int_{J_{i}} d x=\frac{H_{0}}{l_{0}}\left\|\hat{l}^{\eta}-l\right\|_{L^{\infty}(0,1)}(f-e) . \\
& \left|I_{e, f}^{5}\right|=\left|\sum_{i=1}^{n} \int_{J_{i}}\left\{\frac{1}{l\left(\hat{x}_{i}\right)} \int_{0}^{l(x)} H(x, s) d s-\frac{1}{l(x)} \int_{0}^{l(x)} H(x, s) d s\right\} d x\right| \\
& \leq\left|\sum_{i=1}^{n} \int_{J_{i}}\left\{\left(\frac{1}{l\left(\hat{x}_{i}\right)}-\frac{1}{l(x)}\right) \int_{0}^{l(x)} H_{0} d s\right\} d x\right| \leq \sum_{i=1}^{n} \int_{J_{i}} \frac{\left|l\left(\hat{x}_{i}\right)-l(x)\right|}{\left|l\left(\hat{x}_{i}\right) l(x)\right|} l(x) H_{0} d x \\
& \leq \frac{l_{1}}{l_{0}^{2}} H_{0}(f-e)\left\|\hat{l}^{\eta}-l\right\|_{L^{\infty}(0,1)} \text {. }
\end{aligned}
$$

Aqui $0 \leq H_{\epsilon} \leq H_{0}=b_{0}+G_{0}$ e $l_{0}, l_{1}$ são constantes positivas introduzidas em (H2). Nas estimativas $I_{e, f}^{4}$ e $I_{e, f}^{5}$ supomos que $J_{i} \stackrel{\text { def }}{=} N_{i} \cup P_{i}$, onde $N_{i}=\bigcup_{i=1}^{n} \alpha_{i}$ onde $\alpha_{i}=\left[\gamma_{i-1}, \gamma_{i}\right]$ é tal que $l\left(\hat{x}_{i}\right)-l(x)>0, \forall x \in \alpha_{i}$ e $P_{i}=\bigcup_{i=1}^{n} \beta_{i}$ onde $\beta_{i}=\left[\delta_{i-1}, \delta_{i}\right]$ é tal que $l\left(\hat{x}_{i}\right)-l(x)<0, \forall x \in \beta_{i}$ e $\hat{l}^{\eta}$ é uma função simples definida para cada $\eta>0$ por

$$
\hat{l}^{\eta}(x)=l\left(x_{i}\right) \quad \text { quando } \quad x_{i} \in J_{i}
$$

Supondo que $x \in \alpha_{i}$ e obtemos estimativas acima que não dependem de $\epsilon>0$ e como $\|\hat{l} \eta-l\|_{L^{\infty}(0,1)} \rightarrow 0$ quando $\eta \rightarrow 0$ uniformemente em $\epsilon$, temos que $I_{e, f}^{1}, I_{e, f}^{3}, I_{e, f}^{4}$ e $I_{e, f}^{5}$ tende para zero quando $\eta \rightarrow 0$ uniformemente em $\epsilon$. Para concluir a prova do lema temos que avaliar $I_{e, f}^{2}$. Mas este é aplicação direta do Teorema A.4.4 desde que $\hat{x}_{i}$ seja ponto fixado em $J_{i}$ e $H\left(\hat{x}_{i}, \cdot\right)$ seja $l\left(\hat{x}_{i}\right)$ - periódico. Portanto

$$
\left|I_{e, f}^{2}\right| \leq \sum_{i=1}^{n} \int_{J_{i}}\left|H\left(\hat{x}_{i}, x / \epsilon^{\alpha}\right)-\frac{1}{l\left(\hat{x}_{i}\right)} \int_{0}^{l\left(\hat{x}_{i}\right)} H\left(\hat{x}_{i}, s\right) d s\right| d x \rightarrow 0, \quad \epsilon \rightarrow 0, \quad L^{\infty}(0,1) .
$$


Afirmação 2.3.2 $\|\hat{\eta} \eta-l\|_{L^{\infty}(0,1)} \rightarrow 0$ uniformemente em $\epsilon$ quando $\eta \rightarrow 0$.

Prova. Consideremos uma partição $\mathcal{P}$ do intervalo $[0,1]$ e definimos as somas superior e inferior de $l$ relativamente a partição qualquer $\mathcal{P}$ por

$$
U(l, \mathcal{P})=\sum_{i} \sup _{x \in\left[x_{i-1}, x_{i}\right]} l(x) \text { e } L(l, \mathcal{P})=\sum_{i} \inf _{x \in\left[x_{i-1}, x_{i}\right]} l(x) .
$$

Pela definição do integral segundo Riemann existe uma partição $\mathcal{P}$ do intervalo $[0,1]$ tal que

$$
\int_{0}^{1} l(x) d x-\frac{\eta}{2} \leq L(l, \mathcal{P}) \leq \int_{0}^{1} l(x) d x \leq U(l, \mathcal{P}) \leq \int_{0}^{1} l(x) d x+\frac{\eta}{2}
$$

em particular

$$
U(l, \mathcal{P})-L(l, \mathcal{P})<\eta
$$

E se a partição $\mathcal{P}$ é definida como no Lema 2.3.1 temos:

$$
\sum_{i=1}^{n}\left(\sup _{x \in J_{i}} l(x)-\inf _{x \in J_{i}} l(x)\right)\left(x_{i}-x_{i-1}\right)<\eta \text {. }
$$

Então, seja

$$
\begin{aligned}
\hat{\imath}:[0,1] & \longrightarrow \mathbb{R} \\
x & \mapsto \begin{cases}l\left(x_{i-1}\right), & \text { se } i \text { é tal que } x \in\left[x_{i-1}, x_{i}[\right. \\
l(f) & , \text { se for o caso contrário. }\end{cases}
\end{aligned}
$$

É claro que $\hat{l}^{\eta}(x)=l\left(x_{i}\right)$ para todo $i$ para todo $x \in J_{i}$ é uma função simples e assim

$$
\begin{aligned}
\operatorname{dist}(l, \hat{l})=\int_{e}^{f}\left|\hat{l}^{\eta}(x)-l(x)\right| d x & =\sum_{i=1}^{n} \int_{J_{i}}\left|\hat{l}^{\eta}(x)-l(x)\right| d x \\
& \leq \sum_{i=1}^{n} \int_{j_{i}}\left(\sup _{x \in J_{i}} l(x)-\inf _{x \in J_{i}} l(x)\right) d x<\eta,
\end{aligned}
$$

por (2.18), onde dist é a função distância e assim podemos escolher um $n$ suficientemente grande de tal forma que

$$
\max _{i}\left(\sup _{x \in J_{i}} l(x)-\inf _{x \in J_{i}} l(x)\right) \rightarrow 0, \quad \text { quando } \quad \eta \rightarrow 0 .
$$

\subsection{Integrais concentradas}

Lema 2.4.1 Suponha que $v \in H^{s}(\Omega) \operatorname{com} \frac{1}{2}<s \leq 1$ e $s-1>-\frac{1}{q}$. Então para $\epsilon_{0}$ suficientemente pequeno existe $C>0$ independente de $\epsilon$ e v tal que para todo $0<\epsilon \leq \epsilon_{0}$ temos que

$$
\frac{1}{\epsilon^{\alpha}} \int_{o_{\epsilon}}|v(\xi)|^{q} d \xi \leq C\|v\|_{H^{s}(\Omega)}^{q}
$$


Prova. Observemos primeiramente que $o_{\epsilon}$ pode ser escrito como

$$
o_{\epsilon}=\left\{\left(x_{1}, x_{2}\right) \in \mathbb{R}^{2}: x_{1} \in(0,1), 0<G\left(x_{1}\right)-x_{2}<\epsilon^{\alpha} H_{\epsilon}\left(x_{1}\right)\right\}
$$

Assim, aplicando o Teorema da Mudança de coordenadas temos

$$
\begin{aligned}
\frac{1}{\epsilon^{\alpha}} \int_{o_{\epsilon}}\left|v\left(x_{1}, x_{2}\right)\right|^{q} d x_{2} d x_{1} & =\frac{1}{\epsilon^{\alpha}} \int_{0}^{1} \int_{0}^{\epsilon^{\alpha} H_{\epsilon}\left(\xi_{1}\right)}\left|v\left(\xi_{1}, G\left(\xi_{1}\right)-\xi_{2}\right)\right|^{q}\left|\operatorname{det}\left(\begin{array}{cc}
1 & 0 \\
G^{\prime}\left(\xi_{1}\right) & -1
\end{array}\right)\right| d \xi_{2} d \xi_{1} \\
& =\frac{1}{\epsilon^{\alpha}} \int_{0}^{1} \int_{0}^{\epsilon^{\alpha} H_{\epsilon}\left(\xi_{1}\right)}\left|v\left(\xi_{1}, G\left(\xi_{1}\right)-\xi_{2}\right)\right|^{q} d \xi_{2} d \xi_{1},
\end{aligned}
$$

$\operatorname{com} \xi_{1}$ e $\xi_{2}$ definido por

$$
\left\{\begin{array}{l}
\xi_{1}\left(x_{1}, x_{2}\right)=x_{1} \\
\xi_{2}\left(x_{1}, x_{2}\right)=G\left(x_{1}\right)-x_{2} .
\end{array}\right.
$$

Seja dada a aplicação $\tau: \tilde{o}_{\epsilon} \rightarrow \mathbb{R}^{2}$ definido por $\tau\left(\xi_{1}, \xi_{2}\right)=\left(\xi_{1}, G\left(\xi_{1}\right)-\xi_{2}\right)$ tal que $\tau\left(o_{\epsilon}\right) \subset \tilde{o}_{\epsilon}$ definido por

$$
\tilde{o}_{\epsilon}=\left\{\left(\xi_{1}, \xi_{2}\right) \in \mathbb{R}^{2}: \xi_{1} \in(0,1), 0<\xi_{2}<\epsilon^{\alpha} H_{0}\right\}
$$

Pelo [9, Lema 2.1] existem constante $\epsilon_{0}$ e $C>0$ independente de $\epsilon$ e $\omega=v \circ \tau$ tal que

$$
\begin{aligned}
\frac{1}{\epsilon^{\alpha}} \int_{o_{\epsilon}}\left|v\left(x_{1}, x_{2}\right)\right|^{q} d x_{2} d x_{1} & =\frac{1}{\epsilon^{\alpha}} \int_{0}^{1} \int_{0}^{\epsilon^{\alpha} H_{\epsilon}\left(\xi_{1}\right)}\left|v\left(\xi_{1}, G\left(\xi_{1}\right)-\xi_{2}\right)\right|^{q} d \xi_{2} d \xi_{1} \\
& \leq \frac{1}{\epsilon^{\alpha}} \int_{0}^{1} \int_{0}^{\epsilon^{\alpha} H_{0}}\left|v \circ \tau\left(\xi_{1}, \xi_{2}\right)\right|^{q} d \xi_{2} d \xi_{1}=\frac{1}{\epsilon^{\alpha}} \int_{0}^{1} \int_{0}^{\epsilon^{\alpha} H_{0}}\left|\omega\left(\xi_{1}, \xi_{2}\right)\right|^{q} d \xi_{2} d \xi_{1} \\
& =\frac{1}{\epsilon^{\alpha}} \int_{\tilde{o}_{\epsilon}}\left|\omega\left(\xi_{1}, \xi_{2}\right)\right|^{q} d \xi_{2} d \xi_{1} \leq C\|\omega\|_{H^{s}(\widetilde{\Omega})}^{q} \quad \forall \epsilon \in\left(0, \epsilon_{0}\right] .
\end{aligned}
$$

Aqui

$$
\widetilde{\Omega}=\left\{\left(\xi_{1}, \xi_{2}\right) \in \mathbb{R}^{2}: \xi_{1} \in(0,1), 0<\xi_{2}<G\left(\xi_{1}\right)+b\left(\xi_{1}\right)\right\}
$$

É claro que para $\epsilon_{0}$ suficientemente pequeno $\tau\left(o_{\epsilon}\right) \subset \widetilde{\Omega}$. Além disso a matriz jacobiana

$$
J_{\tau}\left(\xi_{1}, \xi_{2}\right)=\left(\begin{array}{cc}
1 & 0 \\
G^{\prime}\left(\xi_{1}\right) & -1
\end{array}\right)
$$

independente de $\epsilon$. Para completar a prova do Lema temos que mostrar que existe uma constante $C$ independente de $\epsilon$ tal que $\|\cdot\|_{H^{1}(\widetilde{\Omega})} \leq C\|\cdot\|_{H^{1}(\Omega)}$. Pelo que

Definição 2.4.2 Seja $U \subset \mathbb{R}^{n}$ um aberto diremos que aplicação $\varphi: U \rightarrow \mathbb{R}^{n}$ é um mergulho $\alpha$ vezes diferenciáveis se $\varphi$ for um defeomorfismo de U sobre a sua imagem $\varphi(U)$ de ordem $\alpha$. Definimos a aplicação composição (ou pull-back) $\varphi^{*}$ de $\varphi$

$$
\varphi^{*} h(x)=h \circ \varphi(x)=h(\varphi(x)), \quad \forall x \in U,
$$


onde h é uma função definida em $U$. Mostra-se que $\varphi^{*}: C^{\alpha}(\varphi(U)) \rightarrow C^{\alpha}(U)$ é um isomorfismo com a inversa $\varphi^{*-1}=\left(\varphi^{-1}\right)^{*}[6]$. Esta definição é válida em outros espaços com por exemplo nos espaços de Sobolev $\varphi^{*}: W^{m, p}(\varphi(U)) \rightarrow W^{m, p}(U) \operatorname{com} 0 \leq m \leq \alpha$ e $1 \leq p \leq \infty$.

Definimos a seguinte aplicação

$$
\begin{aligned}
\tau^{*}: H^{s}(\widetilde{\Omega}) & \rightarrow H^{s}(\Omega) \\
v & \mapsto v \circ \tau
\end{aligned}
$$

onde $\tau: \Omega \rightarrow \widetilde{\Omega}$ é um mergulho. Então se $\omega=\tau^{*} v \in H^{s}(\Omega)$ pela regra da cadeia temos que $v=\omega \circ \tau^{-1} \in H^{s}(\widetilde{\Omega})$, portanto $\tau^{*}$ está bem definida. Assim para avaliarmos as normas vamos dividir em dois casos a saber:

(1) $s=1, \omega \in H^{1}(\widetilde{\Omega})$ então pela Regra da Cadeia temos:

$$
\partial_{i}\left(\omega\left(\xi_{1}, \xi_{2}\right)\right)=\partial_{i}\left(\tau^{*} v\left(\xi_{1}, \xi_{2}\right)\right)=\partial_{i}\left(v \circ \tau\left(\xi_{1}, \xi_{2}\right)\right)=\sum_{j}^{2} \partial_{j}\left(v\left(\tau\left(\xi_{1}, \xi_{2}\right)\right)\right) \partial_{i}\left(\tau\left(\xi_{1}, \xi_{2}\right)\right)_{j}
$$

e a norma em $H^{1}(\widetilde{\Omega})$ é dado por

$$
\|\omega\|_{H^{1}(\widetilde{\Omega})}^{2}=\|\omega\|_{L^{2}(\widetilde{\Omega})}^{2}+\left\|\partial_{1} \omega\right\|_{L^{2}(\widetilde{\Omega})}^{2}+\left\|\partial_{2} \omega\right\|_{L^{2}(\widetilde{\Omega})}^{2} .
$$

Portanto, vamos estimar cada uma das integrais no segundo membro utilizando a substituição dada em (2.19)

$$
\begin{aligned}
\|\omega\|_{L^{2}(\widetilde{\Omega})}^{2} & =\int_{\widetilde{\Omega}}\left|\omega\left(\xi_{1}, \xi_{2}\right)\right|^{2} d \xi_{2} d \xi_{1}=\int_{0}^{1} \int_{0}^{G\left(\xi_{1}\right)+b\left(\xi_{1}\right)}\left|v \circ \tau\left(\xi_{1}, \xi_{2}\right)\right|^{2} d \xi_{2} d \xi_{1} \\
& =\int_{0}^{1} \int_{0}^{G\left(\xi_{1}\right)+b\left(\xi_{1}\right)}\left|v\left(\xi_{1}, G\left(\xi_{1}\right)-\xi_{2}\right)\right|^{2} d \xi_{2} d \xi_{1}=\int_{0}^{1} \int_{-b\left(y_{1}\right)}^{G\left(y_{1}\right)}\left|v\left(y_{1}, y_{2}\right)\right|^{2}\left|J_{\tau^{-1}}\left(y_{1}, y_{2}\right)\right| d y_{2} d y_{1} \\
& =\int_{\Omega}\left|v\left(y_{1}, y_{2}\right)\right|^{2} d y_{2} d y_{1}=\|v\|_{L^{2}(\Omega)}^{2} . \\
\left\|\partial_{1} \omega\right\|_{L^{2}(\widetilde{\Omega})}^{2} & =\int_{\widetilde{\Omega}}\left|\partial_{1} \omega\left(\xi_{1}, \xi_{2}\right)\right|^{2} d \xi_{2} d \xi_{1}=\int_{0}^{1} \int_{0}^{G\left(\xi_{1}\right)+b\left(\xi_{1}\right)}\left|\partial_{1} v \circ \tau\left(\xi_{1}, \xi_{2}\right)\right|^{2} d \xi_{2} d \xi_{1} \\
& =\int_{0}^{1} \int_{0}^{G\left(\xi_{1}\right)+b\left(\xi_{1}\right)}\left|\partial_{1} v\left(\xi_{1}, G\left(\xi_{1}\right)-\xi_{2}\right)\right|^{2} d \xi_{2} d \xi_{1} \\
& =\int_{0}^{1} \int_{0}^{G\left(\xi_{1}\right)+b\left(\xi_{1}\right)}\left|\partial_{1} v\left(\xi_{1}, G\left(\xi_{1}\right)-\xi_{2}\right) \cdot 1+\partial_{1} v\left(\xi_{1}, G\left(\xi_{1}\right)-\xi_{2}\right) \cdot G^{\prime}\left(\xi_{1}\right)\right|^{2} d \xi_{2} d \xi_{1} \\
& =\int_{0}^{1} \int_{0}^{G\left(\xi_{1}\right)+b\left(\xi_{1}\right)}\left|\partial_{1} v\left(\xi_{1}, G\left(\xi_{1}\right)-\xi_{2}\right)\left[1+G^{\prime}\left(\xi_{1}\right)\right]\right|^{2} d \xi_{2} d \xi_{1} \\
& \leq\left[1+\left\|G^{\prime}\right\|_{L^{\infty}(0,1)}\right]^{2} \int_{0}^{1} \int_{-b\left(y_{1}\right)}^{G\left(y_{1}\right)}\left|\partial_{1} v\left(y_{1}, y_{2}\right)\right|^{2}\left|J_{\tau^{-1}}\left(y_{1}, y_{2}\right)\right| d y_{2} d y_{1} \\
& =\left[1+\left\|G^{\prime}\right\|_{L^{\infty}(0,1)}\right]^{2} \int_{\Omega}\left|\partial_{1} v\left(y_{1}, y_{2}\right)\right|^{2} d y_{2} d y_{1}=\left[1+\left\|G^{\prime}\right\|_{L^{\infty}(0,1)}\right]^{2}\left\|\partial_{1} v\right\|_{L^{2}(\Omega)}^{2} .
\end{aligned}
$$




$$
\begin{aligned}
\left\|\partial_{2} \omega\right\|_{L^{2}(\widetilde{\Omega})}^{2} & =\int_{\widetilde{\Omega}}\left|\partial_{2} \omega\left(\xi_{1}, \xi_{2}\right)\right|^{2} d \xi_{2} d \xi_{1}=\int_{0}^{1} \int_{0}^{G\left(\xi_{1}\right)+b\left(\xi_{1}\right)}\left|\partial_{2} v \circ \tau\left(\xi_{1}, \xi_{2}\right)\right|^{2} d \xi_{2} d \xi_{1} \\
& =\int_{0}^{1} \int_{0}^{G\left(\xi_{1}\right)+b\left(\xi_{1}\right)}\left|\partial_{2} v\left(\xi_{1}, G\left(\xi_{1}\right)-\xi_{2}\right)\right|^{2} d \xi_{2} d \xi_{1} \\
& =\int_{0}^{1} \int_{-b\left(y_{1}\right)}^{G\left(y_{1}\right)}\left|\partial_{1} v\left(y_{1}, y_{2}\right)\right|^{2}\left|J_{\tau^{-1}}\left(y_{1}, y_{2}\right)\right| d y_{2} d y_{1}=\int_{\Omega}\left|\partial_{1} v\left(y_{1}, y_{2}\right)\right|^{2} d y_{2} d y_{1} \\
& =\left\|\partial_{2} v\right\|_{L^{2}(\Omega)}^{2} .
\end{aligned}
$$

Logo, por (2.20), (2.21) e (2.22) e e tomando $C_{1}:=\left[1+\left\|G^{\prime}\right\|_{L^{\infty}(0,1)}\right]^{2}$ facilmente vemos

$$
\|\omega\|_{H^{1}(\widetilde{\Omega})}^{2} \leq C_{1}\|v\|_{H^{1}(\Omega)}^{2}
$$

(2) Para $\frac{1}{2}<s<1$ dado $\omega \in H^{s}(\widetilde{\Omega})$ temos:

$$
\begin{aligned}
\|\omega\|_{H^{s}(\widetilde{\Omega})}^{2} & =\|\omega\|_{L^{2}(\widetilde{\Omega})}^{2}+\iint_{\widetilde{\Omega} \times \widetilde{\Omega}} \frac{\left|\omega\left(\xi_{1}, \xi_{2}\right)-\omega\left(y_{1}, y_{2}\right)\right|^{2}}{\left|\left(\xi_{1}, \xi_{2}\right)-\left(y_{1}, y_{2}\right)\right|^{2(s+1)}} d y_{2} d y_{1} d \xi_{2} d \xi_{1} \\
& =\|v\|_{L^{2}(\Omega)}^{2}+\iint_{\widetilde{\Omega} \times \widetilde{\Omega}} \frac{\left.\mid \tau^{*} v\left(\xi_{1}, \xi_{2}\right)-\tau^{*} v\left(y_{1}, y_{2}\right)\right)\left.\right|^{2}}{\left|\left(\xi_{1}, \xi_{2}\right)-\left(y_{1}, y_{2}\right)\right|^{2(s+1)}} d y_{2} d y_{1} d \xi_{2} d \xi_{1} \\
& =\|v\|_{L^{2}(\Omega)}^{2}+\iint_{\widetilde{\Omega} \times \widetilde{\Omega}} \frac{\left|v\left(\tau\left(\xi_{1}, \xi_{2}\right)\right)-v\left(\tau\left(y_{1}, y_{2}\right)\right)\right|^{2}}{\left|\tau\left(\xi_{1}, \xi_{2}\right)-\tau\left(y_{1}, y_{2}\right)\right|^{2(s+1)}}\left(\frac{\left|\tau\left(\xi_{1}, \xi_{2}\right)-\tau\left(y_{1}, y_{2}\right)\right|^{2}}{\left|\left(\xi_{1}, \xi_{2}\right)-\left(y_{1}, y_{2}\right)\right|^{2}}\right)^{(s+1)} d y_{2} d y_{1} d \xi_{2} d \xi_{1} \\
& \leq\|v\|_{L^{2}(\Omega)}^{2}+K \iint_{\widetilde{\Omega} \times \widetilde{\Omega}} \frac{\left|v\left(\tau\left(\xi_{1}, \xi_{2}\right)\right)-v\left(\tau\left(y_{1}, y_{2}\right)\right)\right|^{2}}{\left|\tau\left(\xi_{1}, \xi_{2}\right)-\tau\left(y_{1}, y_{2}\right)\right|^{2(s+1)}} d y_{2} d y_{1} d \xi_{2} d \xi_{1} \\
& =\|v\|_{L^{2}(\Omega)}^{2}+K \iint_{\Omega \times \Omega} \frac{\left|v\left(\sigma_{1}, \sigma_{2}\right)-v\left(\varrho_{1}, \varrho_{2}\right)\right|^{2}}{\left|\left(\sigma_{1}, \sigma_{2}\right)-\left(\varrho_{1}, \varrho_{2}\right)\right|^{2(s+1)}}\left|J_{\tau^{-1}}\left(\sigma_{1}, \sigma_{2}\right) J_{\tau^{-1}}\left(\varrho_{1}, \varrho_{2}\right)\right| d \varrho_{1} d \varrho_{2} d \sigma_{2} d \sigma_{1} \\
& =\|v\|_{L^{2}(\Omega)}^{2}+K \iint_{\Omega \times \Omega} \frac{\left|v\left(\sigma_{1}, \sigma_{2}\right)-v\left(\varrho_{1}, \varrho_{2}\right)\right|^{2}}{\left|\left(\sigma_{1}, \sigma_{2}\right)-\left(\varrho_{1}, \varrho_{2}\right)\right|^{2(s+1)}} d \varrho_{1} d \varrho_{2} d \sigma_{2} d \sigma_{1} .
\end{aligned}
$$

Denotando por $C_{2}:=K$ temos que $\|\omega\|_{H^{s}(\widetilde{\Omega})}^{2} \leq C_{2}\|v\|_{H^{s}(\Omega)}^{2}$ para $\frac{1}{2}<s<1$. Assim para todo intervalo $\frac{1}{2}<s \leq 1$ e tomando uma constante $C>0$ arbitrária independente de $\epsilon \omega$ e $v$ temos que $\|\omega\|_{H^{s}(\widetilde{\Omega})}^{2} \leq$ $C\|v\|_{H^{s}(\Omega)}^{2}$. O que implica que $\|\omega\|_{H^{s}(\widetilde{\Omega})} \leq \sqrt{C}\|v\|_{H^{s}(\Omega)}$, donde elevando a $q$ em ambos membros obtemos

$$
\|\omega\|_{H^{s}(\widetilde{\Omega})}^{q} \leq C^{1 / q}\|v\|_{H^{s}(\Omega)}^{q} \cdot
$$

Afirmação 2.4.3 A aplicação $\tau: \tilde{o}_{\epsilon} \rightarrow \mathbb{R}^{2}$ definida por $\tau\left(\xi_{1}, \xi_{2}\right)=\left(x_{1}, G\left(x_{1}\right)-x_{2}\right)$ é Lipschitz contínua, isto é, existe uma constante positiva tal que:

$$
\left|\tau\left(\xi_{1}, \xi_{2}\right)-\tau\left(y_{1}, y_{2}\right)\right| \leq K\left|\left(\xi_{1}, \xi_{2}\right)-\left(y_{1}, y_{2}\right)\right|
$$


onde $K=\left\{1+2\left\|G^{\prime}\right\|_{L^{\infty}(0,1)}^{2}\right\}^{1 / 2}$.

Prova. Temos que $\left|\tau\left(\xi_{1}, \xi_{2}\right)-\tau\left(y_{1}, y_{2}\right)\right|=\left|\left(x_{1}-y_{1}\right),\left(G\left(x_{1}\right)-G\left(y_{1}\right)-\left(x_{2}-y_{2}\right)\right)\right|$. Elevando ambos membros ao quadrado obtemos que

$$
\begin{aligned}
\left|\tau\left(\xi_{1}, \xi_{2}\right)-\tau\left(y_{1}, y_{2}\right)\right|^{2} & =\left|\left(x_{1}-y_{1}\right),\left(G\left(x_{1}\right)-G\left(y_{1}\right)-\left(x_{2}-y_{2}\right)\right)\right|^{2} \\
& =\left(x_{1}-y_{1}\right)^{2}+\left(G\left(x_{1}\right)-G\left(y_{1}\right)\right)^{2}-2\left(G\left(x_{1}\right)-G\left(y_{1}\right)\right)\left(x_{2}-y_{2}\right)+\left(x_{2}-y_{2}\right)^{2} \\
& =\left|\left(\xi_{1}, \xi_{2}\right)-\left(y_{1}, y_{2}\right)\right|^{2}+\left(G\left(x_{1}\right)-G\left(y_{1}\right)\right)^{2}+2\left(G\left(x_{1}\right)-G\left(y_{1}\right)\right)\left(y_{2}-x_{2}\right) .
\end{aligned}
$$

Aplicando o Teorema de Valor Médio para $G\left(x_{1}\right)-G\left(y_{1}\right)$, com $y_{1}<\theta<x_{1}$ obtemos que

$$
\left|G\left(x_{1}\right)-G\left(y_{1}\right)\right|=\left|G^{\prime}(\theta)\left(x_{1}-y_{1}\right)\right| \leq\left\|G^{\prime}\right\|_{L^{\infty}(0,1)}\left|x_{1}-y_{1}\right| .
$$

Agora usando os seguintes fatos: $2 a b \leq a^{2}+b^{2}$ e se $a \leq b$ então $a \leq b+c \operatorname{com} c \geq 0$. Assim

$$
\begin{aligned}
\left|\tau\left(\xi_{1}, \xi_{2}\right)-\tau\left(y_{1}, y_{2}\right)\right|^{2} & \leq\left|\left(\xi_{1}, \xi_{2}\right)-\left(y_{1}, y_{2}\right)\right|^{2}+\left\|G^{\prime}\right\|_{L^{\infty}(0,1)}^{2}\left|x_{1}-y_{1}\right|^{2}+2\left\|G^{\prime}\right\|_{L^{\infty}(0,1)}\left|x_{1}-y_{1} \| x_{2}-y_{2}\right|^{2} \\
& \leq\left|\left(\xi_{1}, \xi_{2}\right)-\left(y_{1}, y_{2}\right)\right|^{2}+\left\|G^{\prime}\right\|_{L^{\infty}(0,1)}^{2}\left|x_{1}-y_{1}\right|^{2}+\left\|G^{\prime}\right\|_{L^{\infty}(0,1)}\left(\left|x_{1}-y_{1}\right|^{2}+\left|x_{2}-y_{2}\right|^{2}\right) \\
& \leq\left|\left(\xi_{1}, \xi_{2}\right)-\left(y_{1}, y_{2}\right)\right|^{2}+\left\|G^{\prime}\right\|_{L^{\infty}(0,1)}^{2}\left|x_{1}-y_{1}\right|^{2}+\left\|G^{\prime}\right\|_{L^{\infty}(0,1)}^{2}\left(\left|x_{1}-y_{1}\right|^{2}+\left|x_{2}-y_{2}\right|^{2}\right) \\
& +\left\|G^{\prime}\right\|_{L^{\infty}(0,1)}^{2}\left|x_{2}-y_{2}\right|^{2}=\left|\left(\xi_{1}, \xi_{2}\right)-\left(y_{1}, y_{2}\right)\right|^{2}+2\left\|G^{\prime}\right\|_{L^{\infty}(0,1)}^{2}\left(\left|x_{1}-y_{1}\right|^{2}+\left|x_{2}-y_{2}\right|^{2}\right) \\
& =\left|\left(\xi_{1}, \xi_{2}\right)-\left(y_{1}, y_{2}\right)\right|^{2}\left(1+2\left\|G^{\prime}\right\|_{L^{\infty}(0,1)}^{2}\right)
\end{aligned}
$$

E assim mostramos que $\left|\tau\left(\xi_{1}, \xi_{2}\right)-\tau\left(y_{1}, y_{2}\right)\right|^{2} \leq\left(1+2\left\|G^{\prime}\right\|_{L^{\infty}(0,1)}^{2}\right)\left|\left(\xi_{1}, \xi_{2}\right)-\left(y_{1}, y_{2}\right)\right|^{2}$.

Com base no Lema 2.4.1, podemos analizar como integrais concentrados convergem para uma certa família de funções que variam com $\epsilon$ e termos as propriedades de regularidade fraca. Mas precisamente

Lema 2.4.4 Suponha que as sequências $\phi^{\epsilon}, \varphi^{\epsilon} \in H_{\epsilon}^{1}(\Omega)$ são uniformemente limitadas em $\epsilon>0$, e $\phi, \varphi \in$ $H^{1}(0,1)$ satisfazendo $\phi^{\epsilon} \rightarrow \phi e \varphi^{\epsilon} \rightarrow \varphi, w-H^{1}(\Omega)$. Então

$$
\lim _{\epsilon \rightarrow 0} \frac{1}{\epsilon^{\alpha}} \int_{o_{\epsilon}} \phi^{\epsilon} \varphi^{\epsilon} d \xi=\int_{0}^{1} \mu \phi \varphi d S,
$$

onde $\mu \in L^{\infty}(0,1)$ é dado em (1.6).

Prova. É facil ver que

$$
\begin{aligned}
\frac{1}{\epsilon^{\alpha}} \int_{o_{\epsilon}} \phi^{\epsilon} \varphi^{\epsilon} d \xi-\int_{0}^{1} \mu \phi \varphi d S & =\frac{1}{\epsilon^{\alpha}} \int_{o_{\epsilon}}\left(\phi^{\epsilon}\left(x_{1}, x_{2}\right)-\phi\left(x_{1}\right)\right) \varphi^{\epsilon}\left(x_{1}, x_{2}\right) d x_{2} d x_{1} \\
& +\frac{1}{\epsilon^{\alpha}} \int_{o_{\epsilon}} \phi\left(x_{1}\right)\left(\varphi^{\epsilon}\left(x_{1}, x_{2}\right)-\varphi\left(x_{1}\right)\right) d \xi_{2} d \xi_{1}+\frac{1}{\epsilon^{\alpha}} \int_{o_{\epsilon}} \phi\left(x_{1}\right) \varphi\left(x_{1}\right) d x_{2} d x_{1} \\
& -\int_{0}^{1} \mu\left(x_{1}\right) \phi\left(x_{1}\right) \varphi\left(x_{1}\right) d x_{1}
\end{aligned}
$$


Mas,

$$
\frac{1}{\epsilon^{\alpha}} \int_{o_{\epsilon}} \phi\left(x_{1}\right) \varphi\left(x_{1}\right) d x_{2} d x_{1}=\frac{1}{\epsilon^{\alpha}} \int_{0}^{1} \phi\left(x_{1}\right) \varphi\left(x_{1}\right)\left(\int_{G\left(x_{1}\right)-\epsilon^{\alpha} H_{\epsilon}\left(x_{1}\right)}^{G\left(x_{1}\right)} d x_{2}\right)=\int_{0}^{1} \phi\left(x_{1}\right) \varphi\left(x_{1}\right) H_{\epsilon}\left(x_{1}\right) d x_{1} .
$$

Portanto, (2.24) pode ser escrito como

$$
\begin{aligned}
\frac{1}{\epsilon^{\alpha}} \int_{o_{\epsilon}} \phi^{\epsilon} \varphi^{\epsilon} d \xi-\int_{0}^{1} \mu \phi \varphi d S & =\frac{1}{\epsilon^{\alpha}} \int_{o_{\epsilon}}\left(\phi^{\epsilon}\left(x_{1}, x_{2}\right)-\phi\left(x_{1}\right) \varphi^{\epsilon}\left(x_{1}, x_{2}\right) d x_{2} d x_{1}\right. \\
& +\frac{1}{\epsilon^{\alpha}} \int_{o_{\epsilon}} \phi\left(x_{1}\right)\left(\varphi^{\epsilon}\left(x_{1}, x_{2}\right)-\varphi\left(x_{1}\right)\right) d x_{2} d x_{1} \\
& +\int_{0}^{1} \phi\left(x_{1}\right) \varphi\left(x_{1}\right)\left(H_{\epsilon}\left(x_{1}\right)-\mu\left(x_{1}\right) d x_{1} \stackrel{\text { def }}{=} I_{1}+I_{2}+I_{3},\right.
\end{aligned}
$$

onde cada um dos $I_{i}$, com $i=1,2,3$ representam a primeira a segunda e a terceira integrais respectivamente. Agora vamos avaliar cada uma das integrais $I_{i}, i=1,2,3$ que equivale a mostrar que $I_{i} \rightarrow 0$, quando $\epsilon \rightarrow 0$, aplicando o Lema 2.4.1. Comecemos por $I_{1}$. Temos

$$
\begin{aligned}
\left|I_{1}\right| & \leq \frac{1}{\epsilon^{\alpha}} \int_{o_{\epsilon}}\left|\phi^{\epsilon}\left(x_{1}, x_{2}\right)-\phi\left(x_{1}\right) \| \varphi^{\epsilon}\left(x_{1}, x_{2}\right)\right| d x_{2} d x_{1} \\
& \leq\left(\frac{1}{\epsilon^{\alpha}} \int_{o_{\epsilon}}\left|\varphi^{\epsilon}\left(x_{1}, x_{2}\right)\right| d x_{2} d x_{1}\right)^{1 / 2}\left(\frac{1}{\epsilon^{\alpha}} \int_{o_{\epsilon}}\left|\phi^{\epsilon}\left(x_{1}, x_{2}\right)-\phi\left(x_{1}\right)\right| d x_{2} d x_{1}\right)^{1 / 2} \\
& \leq\left(C\left\|\varphi^{\epsilon}\right\|_{H^{1}(\Omega)}^{2}\right)^{1 / 2}\left(C\left\|\phi^{\epsilon}-\phi\right\|_{H^{s}(\Omega)}^{2}\right)^{1 / 2} \\
& =C\left\|\varphi^{\epsilon}\right\|_{H^{1}(\Omega)}\left\|\phi^{\epsilon}-\phi\right\|_{H^{s}(\Omega)}
\end{aligned}
$$

onde $C$ é uma constante positiva independente de $\epsilon$ e $\frac{1}{2}<s<1$. Como a sequência $\varphi^{\epsilon}$ é uniformemente limitada em $H_{\epsilon}^{1}(\Omega)$ e usando o fato de que $\|\cdot\|_{H^{1}(\Omega)} \leq\|\cdot\|_{H_{\epsilon}^{1}(\Omega)}$ então $\varphi^{\epsilon}$ é uniformemente limitada em $H^{1}(\Omega)$ para todo $\epsilon \in(0,1)$. Também por hipótese $\phi^{\epsilon} \rightarrow \phi, w-H^{1}(\Omega)$, o que implica que $\phi^{\epsilon} \rightarrow \phi, s-H^{s}(\Omega)$ para todo $0 \leq s<1$. Assim devido a (2.25) temos que $I_{1} \rightarrow 0$, quando $\epsilon \rightarrow 0$.

$$
\begin{aligned}
\left|I_{2}\right| & \leq \frac{1}{\epsilon^{\alpha}} \int_{0_{\epsilon}}\left|\phi\left(x_{1}\right) \| \varphi^{\epsilon}\left(x_{1}, x_{2}\right)-\varphi\left(x_{1}\right)\right| d x_{2} d x_{1} \\
& \leq\left(\frac{1}{\epsilon^{\alpha}} \int_{0}^{1}\left|\phi\left(x_{1}\right)\right|^{2}\left(\int_{G\left(x_{1}\right)-\epsilon^{\alpha} H_{\epsilon}\left(x_{1}\right)}^{G\left(x_{1}\right)} d x_{2}\right) d x_{1}\right)^{1 / 2}\left(\frac{1}{\epsilon^{\alpha}}\left|\varphi^{\epsilon}\left(x_{1}, x_{2}\right)-\varphi\left(x_{1}\right)\right|^{2} d x_{2} d x_{1}\right)^{1 / 2} \\
& \leq\left(\frac{1}{\epsilon^{\alpha}} \int_{0}^{1}\left|\phi\left(x_{1}\right)\right|^{2} \epsilon^{\alpha} H_{\epsilon}\left(x_{1}\right) d x_{1}\right)^{1 / 2}\left(C\left\|\varphi^{\epsilon}-\varphi\right\|_{H^{s}(\Omega)}^{2}\right)^{1 / 2} \\
& \leq H_{0}^{1 / 2} C^{1 / 2}\|\phi\|_{H^{1}(0,1)}\left\|\varphi^{\epsilon}-\varphi\right\|_{H^{s}(\Omega)}
\end{aligned}
$$

onde $C$ é uma constante positiva independente de $\epsilon$ e $\frac{1}{2}<s<1$. Por hipótese temos que $\varphi^{\epsilon} \rightarrow \varphi$, $w-H^{1}(\Omega)$, o que implica que $\varphi^{\epsilon} \rightarrow \varphi, s-H^{s}(\Omega)$ para todo $0 \leq s<1$. O que devido a (2.26) temos 
que $I_{2} \rightarrow 0$, quando $\epsilon \rightarrow 0$. Finalmente, pelo Lema 2.3.1, temos que $H_{\epsilon}(\cdot) \rightarrow \mu(\cdot), \quad w^{*}-L^{\infty}(0,1)$. Consequentemente,

$$
I_{3}=\int_{0}^{1} \phi\left(x_{1}\right) \varphi\left(x_{1}\right) H_{\epsilon}\left(x_{1}\right) d x-\int_{0}^{1} \phi\left(x_{1}\right) \varphi\left(x_{1}\right) \mu\left(x_{1}\right) d x \rightarrow 0 \quad \forall \phi \varphi \in L^{1}(0,1) .
$$

Assim concluimos a prova.

Agora vamos analizar a convergência dos termos não lineares concentrados

Lema 2.4.5 Suponha que as sequências $u^{\epsilon}, \varphi^{\epsilon} \in H_{\epsilon}^{1}(\Omega)$ para cada $\epsilon>0$ e $u, \varphi \in H^{1}(0,1)$ satisfazendo que $u^{\epsilon} \rightarrow u, \varphi^{\epsilon} \rightarrow \varphi, e m w-H^{1}(\Omega)$. Então,

$$
\int_{\Omega} f\left(u^{\epsilon}\right) \varphi^{\epsilon} d x_{1} d x_{2} \rightarrow \int_{0}^{1} p f(u) \varphi d x
$$

e

$$
\frac{1}{\epsilon^{\alpha}} \int_{o_{\epsilon}} g\left(u^{\epsilon}\right) \varphi^{\epsilon} d x_{1} d x_{2} \rightarrow \int_{0}^{1} \mu g(u) \varphi d x
$$

quando $\epsilon \rightarrow 0$, onde $\mu$ e p são definidos em (1.6).

Prova. Como $f$ possui derivada limitada, facilmente vemos que $f\left(u^{\epsilon}\right) \rightarrow f(u)$ em $L^{2}(\Omega)$. De fato

$$
\begin{aligned}
\left\|f\left(u^{\epsilon}\right)-f(u)\right\|_{L^{2}(\Omega)}^{2} & =\int_{\Omega}\left|f\left(u^{\epsilon}\left(x_{1}, x_{2}\right)\right)-f\left(u\left(x_{1}\right)\right)\right|^{2} d x_{2} d x_{1} \leq \int_{\Omega}\left|f^{\prime}\left(\theta\left(x_{1}, x_{2}\right)\right)\right|^{2}\left|u^{\epsilon}\left(x_{1}, x_{2}\right)-u\left(x_{1}\right)\right|^{2} d x_{2} d x_{1} \\
& \leq\left\|f^{\prime}\right\|_{L^{\infty}(\mathbb{R})}^{2}\left\|u^{\epsilon}-u\right\|_{H^{1}(\Omega)^{\prime}}^{2}
\end{aligned}
$$

onde $u\left(x_{1}\right) \leq \theta\left(x_{1}, x_{2}\right) \leq u^{\epsilon}\left(x_{1}, x_{2}\right)$ em $\Omega$. Assim,

$$
\left\|f\left(u^{\epsilon}\right)-f(u)\right\|_{L^{2}(\Omega)} \leq\left\|f^{\prime}\right\|_{L^{\infty}(\mathbb{R})}\left\|u^{\epsilon}-u\right\|_{H^{1}(\Omega)} \rightarrow 0 .
$$

Logo

$$
\begin{aligned}
\int_{\Omega} f\left(u^{\epsilon}\left(x_{1}, x_{2}\right)\right) \varphi^{\epsilon}\left(x_{1}, x_{2}\right) d x_{2} d x_{1} & \rightarrow \int_{\Omega} f\left(u\left(x_{1}\right)\right) \varphi\left(x_{1}\right) d x_{2} d x_{1} \\
& =\int_{0}^{1}\left(\int_{-b\left(x_{1}\right)}^{G\left(x_{1}\right)} d x_{2}\right) f\left(u\left(x_{1}\right)\right) \varphi\left(x_{1}\right) d x_{1}=\int_{0}^{1} p\left(x_{1}\right) f\left(u\left(x_{1}\right)\right) \varphi\left(x_{1}\right) d x_{1},
\end{aligned}
$$

ou seja,

$$
\int_{\Omega} f\left(u^{\epsilon}\left(x_{1}, x_{2}\right)\right) \varphi^{\epsilon}\left(x_{1}, x_{2}\right) d x_{2} d x_{1} \rightarrow \int_{0}^{1} p\left(x_{1}\right) f\left(u\left(x_{1}\right)\right) \varphi\left(x_{1}\right) d x_{1},
$$

provando assim a convergência (2.27).

Agora vamos analizar (2.28). Adicionando e subtraindo termos correspondentes obtemos

$$
\begin{aligned}
\frac{1}{\epsilon^{\alpha}} \int_{o_{\epsilon}} g\left(u^{\epsilon}\right) \varphi^{\epsilon} d x_{2} d x_{1}-\int_{0}^{1} \mu g(u) \varphi d x & =\frac{1}{\epsilon^{\alpha}} \int_{o_{\epsilon}} g\left(u^{\epsilon}\right) \varphi^{\epsilon} d x_{2} d x_{1}+\frac{1}{\epsilon^{\alpha}} \int_{o_{\epsilon}} g\left(u^{\epsilon}\right) \varphi d x_{2} d x_{1} \\
& -\frac{1}{\epsilon^{\alpha}} \int_{o_{\epsilon}} g\left(u^{\epsilon}\right) \varphi d x_{2} d x_{1}+\int_{0}^{1} g(u) H_{\epsilon}\left(x_{1}\right) \varphi d x_{1} \\
& -\int_{0}^{1} g(u) H_{\epsilon}\left(x_{1}\right) \varphi d x_{1}-\int_{0}^{1} \mu g(u) \varphi d x_{1} .
\end{aligned}
$$


Temos,

$$
\int_{0}^{1} g\left(u\left(x_{1}\right)\right) H_{\epsilon}\left(x_{1}\right) \varphi\left(x_{1}\right) d x_{1}=\int_{0}^{1} g\left(u\left(x_{1}\right)\right) \varphi\left(x_{1}\right)\left(\int_{G\left(x_{1}\right)-\epsilon^{\alpha} H_{\epsilon}\left(x_{1}\right)}^{G\left(x_{1}\right)} \epsilon^{-\alpha} d x_{2}\right) d x_{1}=\frac{1}{\epsilon^{\alpha}} \int_{\sigma_{\epsilon}} g(u) \varphi d x_{2} d x_{1} .
$$

Agrupando os termos, (2.29) pode ser escrito na seguinte forma

$$
\begin{aligned}
\frac{1}{\epsilon^{\alpha}} \int_{o_{\epsilon}} g\left(u^{\epsilon}\right) \varphi^{\epsilon} d x_{1} d x_{2}-\int_{0}^{1} \mu g(u) \varphi d x & =\frac{1}{\epsilon^{\alpha}} \int_{o_{\epsilon}} g\left(u^{\epsilon}\right)\left[\varphi^{\epsilon}-\varphi\right] d x_{2} d x_{1}+\int_{0}^{1} g(u)\left[H_{\epsilon}\left(x_{1}\right)-\mu\left(x_{1}\right)\right] \varphi d x_{1} \\
& +\frac{1}{\epsilon^{\alpha}} \int_{o_{\epsilon}}\left[g\left(u^{\epsilon}\right)-g(u)\right] \varphi d x_{2} d x_{1} \stackrel{\text { def }}{=} I_{1}+I_{2}+I_{3} .
\end{aligned}
$$

Aqui $I_{i}, i=1,2,3$, representam a primeira, a segunda e a terceira integrais respectivamente. Agora vamos mostrar que $I_{i} \rightarrow 0$ quando $\epsilon \rightarrow 0$, o que implica a convergência (2.28).

$$
\begin{aligned}
\left|I_{1}\right| & \leq \frac{1}{\epsilon^{\alpha}} \int_{o_{\epsilon}}\left|g ( u ^ { \epsilon } ( x _ { 1 } , x _ { 2 } ) ) \left\|\varphi^{\epsilon}\left(x_{1}, x_{2}\right)-\varphi\left(x_{1}\right)\left|d x_{2} d x_{1} \leq\|g\|_{L^{\infty}(\mathbb{R})} \frac{1}{\epsilon^{\alpha}} \int_{o_{\epsilon}}\right| \varphi^{\epsilon}\left(x_{1}, x_{2}\right)-\varphi\left(x_{1}\right) \mid d x_{2} d x_{1}\right.\right. \\
& \leq C\|g\|_{L^{\infty}(\mathbb{R})}\left\|\varphi^{\epsilon}-\varphi\right\|_{H^{s}(\Omega)}
\end{aligned}
$$

para $\frac{1}{2}<s<1$ devido ao Lema 2.4.1. Como $\varphi^{\epsilon} \rightarrow \varphi, w-H^{1}(\Omega)$, implica que $\varphi^{\epsilon} \rightarrow \varphi, s-H^{s}(\Omega)$ para todo $0 \leq s<1$, obtemos que $I_{1} \rightarrow 0$. Segue diretamente do ao Lema 2.3.1 que $I_{2} \rightarrow 0$, quando $\epsilon \rightarrow 0$, para toda $g \varphi \in L^{1}(0,1)$.

Finalmente, para avaliar $I_{3}$ é necessário usar o Teorema de Lagrange (sobre acréscimos finitos), isto é, existe $\zeta$, definida em $u(x)<\zeta\left(x_{1}, x_{2}\right)<u^{\epsilon}\left(x_{1}, x_{2}\right)$ em $\Omega$ tal que $g\left(u^{\epsilon}\right)-g(u)=g^{\prime}(\zeta)\left(u^{\epsilon}\left(x_{1}, x_{2}\right)-\right.$ $\left.u\left(x_{1}\right)\right)$ e portanto,

$$
\begin{aligned}
\left|I_{3}\right| & \leq \frac{1}{\epsilon^{\alpha}} \int_{o_{\epsilon}}\left|g\left(u^{\epsilon}\left(x_{1}, x_{2}\right)\right)-g\left(u\left(x_{1}\right)\right)\left\|\phi\left(x_{1}\right)\left|d x_{2} d x_{1} \leq \frac{1}{\epsilon^{\alpha}} \int_{o_{\epsilon}}\right| g^{\prime}(\zeta)\right\| u^{\epsilon}\left(x_{1}, x_{2}\right)-u\left(x_{1}\right) \| \phi\left(x_{1}\right)\right| d x_{2} d x_{1} \\
& \leq\left\|g^{\prime}\right\|_{L^{\infty}(\mathbb{R})}\left(\frac{1}{\epsilon^{\alpha}} \int_{o_{\epsilon}}\left|\phi\left(x_{1}\right)\right|^{2} d x_{2} d x_{1}\right)^{1 / 2}\left(\frac{1}{\epsilon^{\alpha}} \int_{o_{\epsilon}}\left|u^{\epsilon}\left(x_{1}, x_{2}\right)-u\left(x_{1}\right)\right|^{2} d x_{2} d x_{1}\right)^{1 / 2} \\
& \leq\left\|g^{\prime}\right\|_{L^{\infty}(\mathbb{R})}\|\phi\|_{H^{1}(0,1)}\left(\frac{1}{\epsilon^{\alpha}} \int_{G\left(x_{1}\right)-\epsilon^{\alpha} H_{\epsilon}\left(x_{1}\right)}^{G\left(x_{1}\right)} d x_{2}\right)^{1 / 2}\left(C\left\|u^{\epsilon}-u\right\|_{H^{s}(\Omega)}^{2}\right)^{1 / 2} \\
& \leq H_{0}^{1 / 2} C^{1 / 2}\left\|g^{\prime}\right\|_{L^{\infty}(\mathbb{R})}\|\phi\|_{H^{1}(0,1)}\left\|u^{\epsilon}-u\right\|_{H^{s}(\Omega)}
\end{aligned}
$$

onde $C$ é uma constante positiva independente de $\epsilon$ e $\frac{1}{2}<s<1$, devido ao Lema 2.4.1. Por hipótese temos que $u^{\epsilon} \rightarrow u, w-H^{1}(\Omega)$, o que implica que $u^{\epsilon} \rightarrow u, s-H^{s}(\Omega)$ para todo $0 \leq s<1$. E portanto devido a (2.30) temos que $I_{3} \rightarrow 0$ o que prova a convergência (2.28). 


\section{CAPÍTULO 3}

\section{SEMI-CONTINUIDADE SUPERIOR E INFERIOR DAS SOLUÇÕES}

Neste capítulo pretendemos provar o resultado principal deste trabalho. Pela Observação 1.2.6 os ítens (i) e (ii) do Teorema 1.2.3 implicam na semi-continuidade superior e inferior do conjunto do esquílibrio do problema parabólico associado ao problema (1.7) em $\epsilon=0$. Primeiro vamos provar a semi-continuidade superior e notar que a solução do problema (1.5) é obtida como limite da solução do problema (1.7). Seguidamente, com auxílio do [12, Teorema 3] provar a semi-continuidade inferior.

\subsection{Semi-continuidade superior}

Nesta seção vamos provar o ítem (i) do Teorema 1.2.3 com base na seguinte proposição:

Proposição 3.1.1 Seja u uma família de soluções do problema (1.7) satisfazendo $\left\|u^{\epsilon}\right\|_{L^{\infty}(\Omega)} \leq R$ para alguma constante positiva $R$ independente de $\epsilon$.

Então, existe uma subsequência ainda denotaremos por $u^{\epsilon}$, e uma função $u \in H^{1}(\Omega)$ tal que $\|u\|_{L^{\infty}(\Omega)} \leq R$, dependendo apenas da primeira variável, isto é, $u(x, y)=u(x)$, solução do problema (1.5) tal que

$$
\left\|u^{\epsilon}-u\right\|_{H^{1}(\Omega)} \rightarrow 0, \quad \text { quando } \quad \epsilon \rightarrow 0
$$


Demonstração. Primeiro comecemos por observar que a solução $u^{\epsilon}$ do problema (1.7) satisfazendo $\left\|u^{\epsilon}\right\|_{L^{\infty}(\Omega)} \leq R$ é uniformemente limitda em $H^{1}(\Omega)$ em relação a $\epsilon$. Temos que $u^{\epsilon} \in \mathcal{E}_{\epsilon}$, se e somente se

$$
\int_{\Omega}\left\{\frac{\partial u^{\epsilon}}{\partial x} \frac{\partial \varphi}{\partial x}+\frac{1}{\epsilon^{2}} \frac{\partial u^{\epsilon}}{\partial y} \frac{\partial \varphi}{\partial y}+u^{\epsilon} \varphi\right\} d y d x=\int_{\Omega} f\left(u^{\epsilon}\right) \varphi d y d x+\frac{1}{\epsilon^{\alpha}} \int_{\Omega} \chi_{o_{\epsilon}} g\left(u^{\epsilon}\right) \varphi d y d x,
$$

para toda $\varphi \in H^{1}(\Omega)$. Assim tomando $\varphi=u^{\epsilon}$ em (3.1) obtemos que:

$$
\int_{\Omega}\left\{\left(\frac{\partial u^{\epsilon}}{\partial x}\right)^{2}+\frac{1}{\epsilon^{2}}\left(\frac{\partial u^{\epsilon}}{\partial y}\right)^{2}+\left(u^{\epsilon}\right)^{2}\right\} d y d x=\int_{\Omega} f\left(u^{\epsilon}\right) u^{\epsilon} d y d x+\frac{1}{\epsilon^{2}} \int_{o_{\epsilon}} g\left(u^{\epsilon}\right) u^{\epsilon} d y d x
$$

donde, temos que

$$
\left\|\frac{\partial u^{\epsilon}}{\partial x}\right\|_{L^{2}(\Omega)}^{2}+\frac{1}{\epsilon^{2}}\left\|\frac{\partial u^{\epsilon}}{\partial y}\right\|_{L^{2}(\Omega)}^{2}+\left\|u^{\epsilon}\right\|_{L^{2}(\Omega)}^{2} \leq \int_{\Omega}\left|f\left(u^{\epsilon}\right)\left\|u^{\epsilon}\left|d y d x+\frac{1}{\epsilon^{2}} \int_{o_{\epsilon}}\right| g\left(u^{\epsilon}\right)\right\| u^{\epsilon}\right| d y d x .
$$

Usando a Desigualdade de Hölder e o Lema 2.4.1 obtemos

$$
\begin{aligned}
\left\|\frac{\partial u^{\epsilon}}{\partial x}\right\|_{L^{2}(\Omega)}^{2}+\frac{1}{\epsilon^{2}}\left\|\frac{\partial u^{\epsilon}}{\partial y}\right\|_{L^{2}(\Omega)}^{2}+\left\|u^{\epsilon}\right\|_{L^{2}(\Omega)}^{2} & \leq \sup _{|x|<R}|f(x)| \int_{\Omega}\left|u^{\epsilon}\right| d y d x+\sup _{|x|<R}|g(x)| C\left\|u^{\epsilon}\right\|_{H^{s}(\Omega)} \\
& \leq \sup _{|x|<R}\left|f(x)\left\|\left.\Omega\right|^{1 / 2}\right\| u^{\epsilon}\left\|_{L^{2}(\Omega)}+C \sup _{|x|<R} \mid g(x)\right\| u^{\epsilon} \|_{H^{s}(\Omega)}\right. \\
& \leq\left(\sup _{|x|<R}|f(x) \| \Omega|^{1 / 2}+C \sup _{|x|<R}|g(x)|\right)\left\|u^{\epsilon}\right\|_{H^{1}(\Omega)}
\end{aligned}
$$

onde $C$ é uma constante positiva e independente de $\epsilon$ e usando o fato de que $H^{1}(\Omega) \hookrightarrow L^{2}(\Omega)$, $H^{1}(\Omega) \hookrightarrow H^{s}(\Omega)$ são uma imersões compactas e $\|\cdot\|_{H^{1}(\Omega)} \leq\|\cdot\|_{H_{\epsilon}^{1}(\Omega)}$ para todo $\epsilon \in(0,1)$ temos que existe uma constante $K:=K(f, g, C, R, \Omega)$, também independente de $\epsilon$, tal que

$$
\left\|u^{\epsilon}\right\|_{H_{\epsilon}^{1}(\Omega)}^{2} \leq K\left\|u^{\epsilon}\right\|_{H^{1}(\Omega)} \leq K\left\|u^{\epsilon}\right\|_{H_{\epsilon}^{1}(\Omega)}
$$

donde

$$
\left\|u^{\epsilon}\right\|_{H_{\epsilon}^{1}(\Omega)} \leq K, \quad \epsilon \in(0,1)
$$

Assim,

$$
\left\|\frac{\partial u^{\epsilon}}{\partial x}\right\|_{L^{2}(\Omega)}^{2}+\frac{1}{\epsilon^{2}}\left\|\frac{\partial u^{\epsilon}}{\partial y}\right\|_{L^{2}(\Omega)}^{2}+\left\|u^{\epsilon}\right\|_{L^{2}(\Omega)}^{2} \leq K^{2} \quad \forall \epsilon \in(0,1)
$$

resultando que:

$$
\left\|\frac{\partial u^{\epsilon}}{\partial x}\right\|_{L^{2}(\Omega)}, \quad \frac{1}{\epsilon}\left\|\frac{\partial u^{\epsilon}}{\partial y}\right\|_{L^{2}(\Omega)}, \quad\left\|u^{\epsilon}\right\|_{L^{2}(\Omega)} \leq K \quad \forall \epsilon \in(0,1) .
$$

Consequentemente, devido a (3.2) e pelo Teorema A.1.11 podemos extrair subsequências de soluções ainda denotadas por $u^{\epsilon}$ tais que quando $\epsilon \rightarrow 0$

$$
u^{\epsilon} \rightarrow u, \quad w-H^{1}(\Omega) \quad \text { e } \quad \frac{\partial u^{\epsilon}}{\partial y} \rightarrow 0, s-L^{2}(\Omega),
$$


para algum $u \in H^{1}(\Omega)$. Portanto, segue de (3.3) que $u(x, y)=u(x)$ em $\Omega$, isto é, $u$ não depende da variável $y$ e assim $u \in H^{1}(0,1)$, nisto temos que $\frac{\partial u^{\epsilon}}{\partial y}=0$ q.s em $\Omega$, desde que para toda $\varphi \in C_{0}^{\infty}(\Omega)$

$$
\int_{\Omega} u \frac{\partial \varphi}{\partial y} d y d x=\lim _{\epsilon \rightarrow 0} \int_{\Omega} u^{\epsilon} \frac{\partial \varphi}{\partial y} d y d x=-\lim _{\epsilon \rightarrow 0} \int_{\Omega} \varphi \frac{\partial u^{\epsilon}}{\partial y} d y d x=0
$$

Agora é fácil ver que $u$ satisfaz o problema (1.5). Assim usando o Lema 2.4.5 e (3.3) podemos passar o limite na formulação variacional (3.1) e obtemos

$$
\begin{aligned}
\int_{\Omega}\left\{u_{x} \varphi_{x}+u \varphi\right\} d y d x & =\int_{0}^{1} \int_{-b(x)}^{G(x)} f(u) \varphi d y d x+\frac{1}{\epsilon^{\alpha}} \int_{0}^{1} \int_{G(x)-\epsilon^{\alpha} H_{\epsilon}(x)}^{G(x)} g(u) \varphi d y d x \\
& =\int_{0}^{1} p f(u) \varphi d x+\int_{0}^{1} H_{\epsilon}(x) g(u) \varphi d x
\end{aligned}
$$

donde,

$$
\int_{\Omega}\left\{u_{x} \varphi_{x}+u \varphi\right\} d y d x=\int_{0}^{1} p f(u) \varphi d x+\int_{0}^{1} \mu g(u) \varphi d x \quad \text { sempre que } \varphi \in H^{1}(0,1) .
$$

Uma vez que $u$ e $\varphi$ não dependem em $y$ temos que:

$$
\int_{0}^{1} p\left\{u_{x} \varphi_{x}+u \varphi\right\} d x=\int_{0}^{1} p f(u) \varphi d x+\int_{0}^{1} \mu g(u) \varphi d x
$$

onde as funções $p$ e $\mu$ são dadas em (1.6). Notemos que (3.5) é a formulação variacional do problema (1.5).

À seguir vamos mostrar a convergência forte em $H^{1}(\Omega)$, mostrando a convergência de $H^{1}$ - norma. Para isso vamos também usar o Teorema A.3.2 em $H^{1}(\Omega)$ a norma inferior semi-contínua em relação a convergência fraca, isto é,

$$
\|u\|_{H^{1}(\Omega)} \leq \liminf _{\epsilon}\left\|u^{\epsilon}\right\|_{H^{1}(\Omega)} .
$$

De fato, devido a (3.1), (3.3), (3.5) e (3.6) obtemos

$$
\begin{aligned}
\int_{0}^{1} p\left(\frac{d u}{d x}\right)^{2} d x=\int_{\Omega}|\nabla u|^{2} d y d x & \leq \liminf _{\epsilon \in(0,1)} \int_{\Omega}\left|\nabla u^{\epsilon}\right|^{2} d y d x \leq \limsup _{\epsilon \in(0,1)} \int_{\Omega}\left|\nabla u^{\epsilon}\right|^{2} d y d x \\
& \leq \limsup _{\epsilon \in(0,1)} \int_{\Omega}\left\{\left(\frac{\partial u^{\epsilon}}{\partial x}\right)^{2}+\frac{1}{\epsilon^{2}}\left(\frac{\partial u^{\epsilon}}{\partial y}\right)^{2}\right\} d y d x \\
& \leq-\int_{0}^{1} p u^{2} d x+\int_{0}^{1}\{p f(u)+\mu g(u)\} u d x=\int_{0}^{1} p\left(\frac{d u}{d x}\right)^{2} d x
\end{aligned}
$$

Então $\left\|u^{\epsilon}\right\|_{H^{1}(\Omega)} \rightarrow\|u\|_{H^{1}(\Omega)}$.

Observação 3.1.2 Com base Proposição 3.1.1 vemos que a existência das soluções do Problema (1.5) é obtido como limite das soluções do Problema (1.7). 
De fato, dado $u$ e $\varphi$ nas condições da Proposição 3.1.1 que não depende da segunda variável y. Por (3.5) temos

$$
\int_{0}^{1} p(x)\left\{u_{x}(x) \varphi_{x}(x)+u(x) \varphi(x)\right\} d x=\int_{0}^{1} p(x) f(u) \varphi(x) d x+\int_{0}^{1} \mu(x) g(u) \varphi(x) d x,
$$

equivalentemente,

$$
\int_{0}^{1}\left\{u_{x}(x) \varphi_{x}(x) p(x)+u(x) \varphi(x) p(x)-p(x) f(u)-\mu(x) g(u) \varphi(x)\right\} d x=0 .
$$

pela convexidade do intervalo $(0,1)$, temos pelo Teorema de Grisvard sobre a regularidade de um problema de Neumann que $u \in H^{2}(0,1)$. Logo podemos aplicar o Método de Integração por Partes em $\int_{0}^{1} u_{x} \varphi_{x} p(x) d x$, obtendo

$$
\begin{aligned}
\int_{0}^{1} u_{x}(x) \varphi_{x}(x) p(x) d x & =\int_{0}^{1}\left(p(x) u_{x}(x)\right) \varphi_{x}(x) d x=-\int_{0}^{1}\left(p(x) u_{x}(x)\right)_{x} \varphi(x) d x+\left.p(x) u_{x} \varphi\right|_{x=0} ^{x=1} \\
& =-\int_{0}^{1}\left(p(x) u_{x}(x)\right)_{x} \varphi(x) d x+u_{x}(1) p(1) \varphi(1)-u_{x}(0) p(0) \varphi(0)
\end{aligned}
$$

Deste modo (3.7) pode ser escrito como

$$
\int_{0}^{1}\left\{-\frac{1}{p(x)}\left(p(x) u_{x}\right)_{x}+u-f(u)-\frac{\mu(x)}{p(x)} g(u)\right\} p(x) \varphi(x) d x+u_{x}(1) p(1) \varphi(1)-u_{x}(0) p(0) \varphi(0)=0,
$$

$\forall \varphi \in H^{1}(0,1)$. Então Se $\varphi \in C_{c}^{\infty}(0,1)$, da formulação variacional (3.8) temos que

$$
-\frac{1}{p(x)}\left(p(x) u_{x}\right)_{x}+u-f(u)-\frac{\mu(x)}{p(x)} g(u)=0
$$

Se a função $\varphi$ se anula no interior do intervalo $(0,1)$ então de novo da formulação variacional $(3.8)$ temos

$$
u_{x}(1) p(1) \varphi(1)-u_{x}(0) p(0) \varphi(0)=0, \quad \forall \varphi \in H^{1}(0,1)
$$

desde que $p(1), p(0)$ são positivos e $\varphi(1), \varphi(0)$ seja arbitrário. Donde deduzimos que $u_{x}(0)=u_{x}(1)=0$. E assim obtemos o Problema clássico (1.5), ou seja

$$
\left\{\begin{aligned}
-\frac{1}{p(x)}\left(p(x) u_{x}\right)_{x}+u & =f(u)+\frac{\mu(x)}{p(x)} g(u), \text { em } \\
u_{x}(0)=u_{x}(1) & =0
\end{aligned}\right.
$$

onde

$$
p(x)=b(x)+G(x) \quad \text { e } \quad \mu(x)=\frac{1}{l(x)} \int_{0}^{l(x)} H(x, y) d y
$$




\section{$3.2 \mathscr{P}$-Convergência}

Conforme vimos na seção 2.2 as soluções dos problemas (1.7) e (1.5) são obtidos como pontos fixos das aplicações não lineares $\mathcal{A}_{\epsilon}^{-1} \circ F_{\epsilon}$ e $\mathcal{A}_{0}^{-1} \circ F_{0}$ definidas em $H^{1}(\Omega)$ e $H^{1}(0,1)$. Nesta seção pretendemos desenvolver algumas ferramentas abstratas básicas que podem ser usadas para comparar dois problemas lineares diferentes em espaços diferentes que tem origem na Análise Funcional Numérica. A noção da $\mathscr{P}$-convergência a chamada convergência discreta veja a Teoria Geral em [12] .

Sejam $X$ e $X_{\epsilon}$ dois espaços de Banach e $\mathscr{P}=\left(P_{\epsilon}\right)$, com $0<\epsilon<1$ um sistema (conexão) de operadores $P_{\epsilon}: X \rightarrow X_{\epsilon}$ tais que

$$
\left\|P_{\epsilon} u\right\|_{X_{\epsilon}} \rightarrow\|u\|_{X}, \quad \forall u \in X, \quad \text { quando } \quad \epsilon \rightarrow 0
$$

Definição 3.2.1 Uma sequência de elementos $\left\{u_{\epsilon}\right\}_{\epsilon \in(0,1)} \operatorname{com} u_{\epsilon} \in X_{\epsilon}$ é dita $\mathscr{P}$-convergente para uma função $u \in X$ se e somente se

$$
\left\|u_{\epsilon}-P_{\epsilon} u\right\|_{X_{\epsilon}} \rightarrow 0, \quad \text { quando } \quad \epsilon \rightarrow 0
$$

e escreve-se $u_{\epsilon} \stackrel{\mathscr{P}}{\rightarrow} u$.

Denotando por $N=(0,1)$ e $N^{\prime}$ um subconjunto de $N$, isto é, $N^{\prime} \subseteq N$.

Definição 3.2.2 Uma sequência $\left\{u_{\epsilon}\right\}_{\epsilon \in N}$, com $u_{\epsilon} \in X_{\epsilon}$ é dita $\mathscr{P}$-relativamente compacta se para cada subsequência $\left\{u_{\epsilon^{\prime}}\right\}_{\epsilon^{\prime} \in N^{\prime}}$ de $\left\{u_{\epsilon}\right\}_{\epsilon \in N}$ existe uma subsequência $\left\{u_{\epsilon^{\prime \prime}}\right\}_{\epsilon^{\prime \prime} \in N^{\prime \prime}}\left(N^{\prime \prime} \subseteq N^{\prime}\right)$ e uma função $x \in X$ tal que $u_{\epsilon^{\prime \prime}} \rightarrow$ u e escreve-se $\mathscr{P}$-comp

Definição 3.2.3 Uma sequência $\left\{T_{\epsilon}\right\}$ de operadores lineares e limitados $T_{\epsilon}: X_{\epsilon} \rightarrow X_{\epsilon}$, com $\epsilon \in N$ é dita $\mathscr{P} \mathscr{P}$-convergente para um operador linear e limitado $T: X \rightarrow X$ se e somente se

$$
u_{\epsilon} \stackrel{\mathscr{P}}{\rightarrow} u \quad \Rightarrow \quad T_{\epsilon} u_{\epsilon} \stackrel{\mathscr{P}}{\rightarrow} T u
$$

e escreve-se $T_{\epsilon} \stackrel{\mathscr{P} P}{\rightarrow} T$.

Definição 3.2.4 Uma sequência $\left\{T_{\epsilon}\right\}$ de operadores cpmpactos $T_{\epsilon}: X_{\epsilon} \rightarrow X_{\epsilon}$, com $\epsilon \in N$ é dita compactamente convergente para um operador $T: X \rightarrow X$ se $T_{\epsilon} \stackrel{\mathscr{P} \not{P}}{\rightarrow}$ e se verifica a condição de compacticidade é verificada

$$
u_{\epsilon} \in X_{\epsilon} \quad \operatorname{com}\left\|u_{\epsilon}\right\|_{X_{\epsilon}} \leq K \Rightarrow T_{\epsilon} u_{\epsilon} \quad \text { é } \mathscr{P} \mathscr{P}-\text { comp }
$$

onde Ké uma constante positiva. 


\subsection{Propriedades das aplicações $F_{\epsilon}$}

Para obter a semi-continuidade inferior do problema linearizado, é necessário estudar o comportamentos das aplicações da $F_{\epsilon}$ pelo que tem lugar os seguintes resultados

Lema 3.3.1 Suponhamos que $\frac{1}{2}<s<1$. Então para cada $\epsilon \in\left[0, \epsilon_{0}\right]$ para as aplicações $F_{\epsilon}: H^{1}(\Omega) \rightarrow H^{-s}(\Omega)$ tem-se

(1) Existe uma constante K positiva independente de $\epsilon$ tal que:

$$
\begin{aligned}
\left\|F_{\epsilon}(u)\right\|_{H^{-s}(\Omega)} \leq K & \forall u \in H^{1}(\Omega), \quad \text { e } \quad 0<\epsilon \leq \epsilon_{0} \\
\left\|F_{0}(u)\right\|_{H^{-s}(0,1)} \leq K & \forall u \in H^{1}(0,1), \quad \text { e } \quad \epsilon=0 ;
\end{aligned}
$$

(2) A aplicação $F_{\epsilon}$ é globalmente Lipschitz, uniformente em $\epsilon$;

(3) Suponha que as sequências $u^{\epsilon}, \varphi^{\epsilon} \in H_{\epsilon}^{1}(\Omega)$ para cada $\epsilon>0$ e $u, \varphi \in H^{1}(0,1)$ satisfazendo $\varphi^{\epsilon} \rightarrow \varphi$, $u^{\epsilon} \rightarrow$ uem $w-H^{1}(\Omega)$. Então

$$
\left\langle F_{\epsilon}\left(u^{\epsilon}\right), \varphi^{\epsilon}\right\rangle \rightarrow\left\langle F_{0}(u), \varphi\right\rangle, \quad \text { quando } \quad \epsilon \rightarrow 0
$$

Prova. (1) Para cada $u \in H^{1}(\Omega)$ para cada $\epsilon \in\left[0, \epsilon_{0}\right]$ e $v \in H^{s}(\Omega)$ temos

$$
\left\|F_{\epsilon}(u)\right\|_{H^{-s}(\Omega)}=\sup _{\|v\|_{H^{s}(\Omega)}=1}\left|\left\langle F_{\epsilon}(u), v\right\rangle\right| .
$$

Como as funções $f$ e $g$ são limitadas, pelo Lema 2.4.1 e usando que $H^{s}(\Omega) \hookrightarrow L^{2}(\Omega)$ temos

$$
\begin{aligned}
\left|\left\langle F_{\epsilon}(u), v\right\rangle\right| & \leq|\widehat{F}(u), v\rangle|+|\left\langle\widehat{F}_{\epsilon}(u), v\right\rangle\left|\leq \int_{\Omega}\right| f(u)\left\|v\left|d x_{2} d x_{1}+\frac{1}{\epsilon^{\alpha}} \int_{o_{\epsilon}}\right| g(u)\right\| v \mid d x_{2} d x_{1} \\
& \leq\left(\int_{\Omega}|f(u)|^{2} d x_{2} d x_{1}\right)^{1 / 2}\left(\int_{\Omega}|v|^{2} d x_{2} d x_{1}\right)^{1 / 2}+\left(\frac{1}{\epsilon^{\alpha}} \int_{o_{\epsilon}}|g(u)|^{2} d x_{2} d x_{1}\right)^{1 / 2}\left(\frac{1}{\epsilon^{\alpha}} \int_{o_{\epsilon}}|v|^{2} d x_{2} d x_{1}\right)^{1 / 2} \\
& \leq\|f\|_{L^{\infty}(\mathbb{R})}|\Omega|^{1 / 2}\|v\|_{L^{2}(\Omega)}+\|g\|_{L^{\infty}(\mathbb{R})} H_{0}^{1 / 2} C\|v\|_{H^{s}(\Omega)} \leq\left(\|f\|_{L^{\infty}(\mathbb{R})}|\Omega|^{1 / 2}+C^{1 / 2} H_{0}^{1 / 2}\|g\|_{L^{\infty}(\mathbb{R})}\right)\|v\|_{H^{s}(\Omega)},
\end{aligned}
$$

onde $C>0$ e independente de $\epsilon$. Então existe uma constante positiva $K:=K\left(f, g, \Omega, C, H_{0}\right)$ independente de $\epsilon$ tal que

$$
\left\|F_{\epsilon}(u)\right\|_{H^{-s}(\Omega)} \leq K, \quad \forall \epsilon \in\left(0, \epsilon_{0}\right] .
$$

Para cada $u \in H^{1}(0,1)$, com $\epsilon=0$ e $v \in H^{s}(\Omega)$ temos

$$
\left\|F_{0}(u)\right\|_{H^{-s}(0,1)}=\sup _{\|v\|_{H^{s}(0,1)}=1}\left|\left\langle F_{0}(u), v\right\rangle\right|
$$


Temos

$$
\begin{aligned}
\left|\left\langle F_{0}(u), v\right\rangle\right| & \leq \int_{0}^{1}\left|f(u) p\left(x_{1}\right)+\mu\left(x_{1}\right) g(u)\left\|v\left(x_{1}\right) \mid d x_{1} \leq\left(\int_{0}^{1}\left|f(u) p\left(x_{1}\right)+\mu\left(x_{1}\right) g(u)\right|^{2} d x_{1}\right)^{1 / 2}\right\| v \|_{L^{2}(\Omega)}\right. \\
& \leq\left\{2 \int_{0}^{1}|f(u)|^{2} p\left(x_{1}\right)^{2} d x_{1}+2 \int_{0}^{1}|g(u)|^{2}\left|\mu^{2}\left(x_{1}\right)\right| d x_{1}\right\}^{1 / 2}\|v\|_{L^{2}(0,1)} \\
& \leq \sqrt{2}\left\{\mid f\left\|_{L^{\infty}(\mathbb{R})}^{2} H_{1}^{2}+\right\| \mu\left\|_{L^{\infty}(0,1)}^{2}\right\| g \|_{L^{\infty}(\mathbb{R})}^{2}\right\}^{1 / 2}\|v\|_{H^{s}(0,1)} .
\end{aligned}
$$

Portanto existe uma constante positiva $K:=K(f, g, \Omega, p, \mu)$ tal que $\left\|F_{0}(u)\right\|_{H^{-s}(0,1)} \leq K$, quando $\epsilon=0$. (2) Para qualquer $u, \omega \in H^{1}(\Omega)$ e $0<\epsilon \leq \epsilon_{0}$ temos

$$
\left\|F_{\epsilon}(u)-F_{\epsilon}(\omega)\right\|_{H^{-s}(\Omega)}=\sup _{\|v\|_{H^{s}(\Omega)}=1}\left|\left\langle F_{\epsilon}(u)-F_{\epsilon}(\omega), v\right\rangle\right| .
$$

Assim para cada $\epsilon \in\left(0, \epsilon_{0}\right]$ e $v \in H^{s}(\Omega)$ e como as funções $f$ e $g$ possuem derivadas limitadas temos pelo Lema 2.4.1 e o Teorema de Valor Médio que

$$
\begin{aligned}
\left|\left\langle F_{\epsilon}(u)-F_{\epsilon}(\omega), v\right\rangle\right| & \leq|\widehat{F}(u)-\widehat{F}(\omega), v\rangle|+|\left\langle\widehat{F}_{\epsilon}(u)-\widehat{F}_{\epsilon}(\omega), v\right\rangle \mid \\
& \leq \int_{\Omega}\left|f(u)-f(\omega)\left\|v\left|d x_{2} d x_{1}+\frac{1}{\epsilon^{2}} \int_{o_{\epsilon}}\right| g(u)-g(\omega)\right\| v\right| d x_{2} d x_{1} \\
& \leq\left(\int_{\Omega}|f(u)-f(\omega)|^{2} d x_{2} d x_{1}\right)^{1 / 2}\|v\|_{L^{2}(\Omega)}+\left(\frac{1}{\epsilon^{\alpha}} \int_{o_{\epsilon}}|g(u)-g(\omega)|^{2} d x_{2} d x_{1}\right)^{1 / 2} C^{1 / 2}\|v\|_{H^{s}(\Omega)} \\
& \leq\left(\int_{\Omega}\left|f^{\prime}(\zeta)\right|^{2}|u-\omega|^{2} d x_{2} d x_{1}\right)^{1 / 2}\|v\|_{H^{s}(\Omega)}+\left(\frac{1}{\epsilon} \int_{o_{\epsilon}}\left|g^{\prime}(\zeta)\right|^{2}|u-\omega|^{2} d x_{2} d x_{1}\right)^{1 / 2} C^{1 / 2}\|v\|_{H^{s}(\Omega)} \\
& \leq\left(\left\|f^{\prime}\right\|_{L^{\infty}(\mathbb{R})}+C\left\|g^{\prime}\right\|_{L^{\infty}(\mathbb{R})}\right)\|u-\omega\|_{H^{s}(\Omega)}\|v\|_{H^{s}(\Omega)} .
\end{aligned}
$$

Aqui $\zeta:=\zeta\left(x_{1}, x_{2}\right)=\omega\left(x_{1}, x_{2}\right)+\theta\left(x_{1}, x_{2}\right)\left(u\left(x_{1}, x_{2}\right)-\omega\left(x_{1}, x_{2}\right)\right)$ e $0<\theta\left(x_{1}, x_{2}\right)<1$ para todo $x_{1}, x_{2} \in \bar{\Omega}$. Portanto existe uma constante positiva $K:=K\left(f^{\prime}, C, g^{\prime}\right)$ independente de $\epsilon$ tal que

$$
\left\|F_{\epsilon}(u)-F_{\epsilon}(\omega)\right\|_{H^{-s}(\Omega)} \leq K\|u-\omega\|_{H^{s}(\Omega)}
$$

o que implica que para $0<\epsilon \leq \epsilon_{0}, F_{\epsilon}$ é globalmente Lipschitz uniformemente em $\epsilon$.

Para cada $u, \omega \in H^{1}(0,1)$ e $\epsilon=0$ temos

$$
\left\|F_{0}(u)-F_{0}(\omega)\right\|_{H^{-s}(0,1)}=\sup _{\|v\|_{H^{s}(0,1)}=1}\left|\left\langle F_{0}(u)-F_{0}(\omega), v\right\rangle\right|, \quad v \in H^{s}(0,1) .
$$


Donde pelo Teorema de Valor Médio temos

$$
\begin{aligned}
\left|\left\langle F_{0}(u)-F_{0}(\omega), v\right\rangle\right| & \leq \int_{0}^{1}|f(u)-f(\omega)| p\left(x_{1}\right)\left|v\left(x_{1}\right)\right| d x_{1}+\int_{0}^{1}|g(u)-g(\omega)| \mu\left(x_{1}\right)\left|v\left(x_{1}\right)\right| d x_{1} \\
& \leq \int_{0}^{1}\left\{p\left(x_{1}\right)\left|f^{\prime}(\zeta)\right|+\mu\left(x_{1}\right)\left|g^{\prime}(\zeta)\right|\right\}|u-\omega \| v| d x_{1} \\
& \leq\left\{H_{1}\left\|f^{\prime}\right\|_{L^{\infty}(\mathbb{R})}+\|\mu\|_{L^{\infty}(0,1)}\left\|g^{\prime}\right\|_{L^{\infty}(\mathbb{R})}\right\}\|u-\omega\|_{L^{2}(0,1)}\|v\|_{L^{2}(0,1)} \\
& \leq\left\{H_{1}\left\|f^{\prime}\right\|_{L^{\infty}(\mathbb{R})}+\|\mu\|_{L^{\infty}(0,1)}\left\|g^{\prime}\right\|_{L^{\infty}(\mathbb{R})}\right\}\|u-\omega\|_{L H^{s}(0,1)}\|v\|_{H^{s}(0,1)} .
\end{aligned}
$$

Donde vemos que existe uma constante positiva $K:=K\left(f^{\prime}, g^{\prime}, H_{1}, \mu\right)$ tal que

$$
\left\|F_{0}(u)-F_{0}(\omega)\right\|_{H^{-s}(0,1)} \leq K\|u-\omega\|_{H^{s}(0,1)}
$$

(3) Segue diretamente do Lema 2.4.5 pelo que

$$
\begin{aligned}
\left\langle F_{\epsilon}\left(u^{\epsilon}\right), \varphi^{\epsilon}\right\rangle & =\left\langle\widehat{F}\left(u^{\epsilon}\right)+\widehat{F}_{\epsilon}\left(u^{\epsilon}\right), \varphi^{\epsilon}\right\rangle=\left\langle\widehat{F}\left(u^{\epsilon}\right), \varphi^{\epsilon}\right\rangle+\left\langle\widehat{F}_{\epsilon}\left(u^{\epsilon}\right), \varphi^{\epsilon}\right\rangle \\
& \leq \int_{\Omega} f\left(u^{\epsilon}\right) \varphi^{\epsilon} d x_{2} d x_{1}+\frac{1}{\epsilon^{\alpha}} \int_{o_{\epsilon}} g\left(u^{\epsilon}\right) \varphi^{\epsilon} \rightarrow \int_{0}^{1} p\left(x_{1}\right) f(u) \varphi\left(x_{1}\right) d x_{1}+\int_{0}^{1} \mu\left(x_{1}\right) g(u) \varphi\left(x_{1}\right) d x_{1} \\
& =\int_{0}^{1}\left\{f(u)+\frac{\mu\left(x_{1}\right)}{p\left(x_{1}\right)} g(u)\right\} p\left(x_{1}\right) \varphi\left(x_{1}\right) d x_{1}=\left\langle F_{0}(u), \varphi\right\rangle .
\end{aligned}
$$

E assim concluimos a prova do lema.

O resultado a seguir é sobre diferenciabilidade à Fréchet da aplicação $F_{\epsilon}: H^{1}(\Omega) \rightarrow H^{-s}(\Omega)$. (veja no Apêndice Definição D.2.18.)

Lema 3.3.2 Suponhamos que $\frac{1}{2}<s<1$. Então, para cada $0 \leq \epsilon \leq \epsilon_{0}, F_{\epsilon}: H^{1}(\Omega) \rightarrow H^{-s}(\Omega)$ é Fréchet diferenciável, uniformemente em $\epsilon$ com a derivada de Fréchet definida por

$$
\begin{aligned}
F_{\epsilon}^{\prime}: H^{1}(\Omega) & \rightarrow \mathcal{L}\left(H^{1}(\Omega), H^{-s}(\Omega)\right. \\
u \mapsto F_{\epsilon}^{\prime}(u): H^{1}(\Omega) & \rightarrow H^{-s}(\Omega) \\
\omega & \mapsto F_{\epsilon}^{\prime}(u) \omega
\end{aligned}
$$

onde $\mathcal{L}\left(H^{1}(\Omega), H^{-s}(\Omega)\right.$ denota o espaço dos operadores lineares e contínuos de $H^{1}(\Omega)$ em $H^{-s}(\Omega)$ e

$$
\begin{aligned}
& \left\langle F_{\epsilon}^{\prime}(u) \omega, \phi\right\rangle:=\int_{\Omega} f^{\prime}(u) \omega \phi d \xi+\frac{1}{\epsilon^{\alpha}} \int_{o_{\epsilon}} g^{\prime}(u) \omega \phi d \xi, \quad \forall \phi \in H^{s}(\Omega) \quad \text { e } 0<\epsilon \leq \epsilon_{0}, \\
& \left\langle F^{\prime}{ }_{0}(u) \omega, \phi\right\rangle:=\int_{0}^{1}\left\{f^{\prime}(u) \omega p(x)+\mu(x) g^{\prime}(u) \omega\right\} \phi d x, \quad \forall \phi \in H^{s}(0,1) \quad e \quad \epsilon=0 .
\end{aligned}
$$

Prova. Como as funções $f, g \in C^{2}(\mathbb{R})$ com derivadas limitadas e $g^{\prime}(v), f^{\prime}(v) \in \mathcal{L}(\mathbb{R}), \forall v \in \mathbb{R}$ e pela linearidade do integral facilmente e o Lema 2.4.1 vemos que $F_{\epsilon}^{\prime}(u) \in \mathcal{L}\left(H^{1}(\Omega), H^{-s}(\Omega)\right.$. 
Agora vamos mostrar que para dado $\eta>0$, existe um $\delta>0$ independente de $\epsilon$ tal que

$$
\left\|F_{\epsilon}\left(u\left(x_{1}\right)+\omega\left(x_{1}\right)\right)-F_{\epsilon}\left(u\left(x_{1}\right)\right)-F_{\epsilon}^{\prime}\left(u\left(x_{1}\right)\right) \omega\right\|_{H^{-s}(\Omega)} \leq \eta\|\omega\|_{H^{1}(\Omega)}, \quad \omega \in H^{1}(\Omega) \quad \text { com } \quad\|\omega\|_{H^{1}(\Omega)} \leq \delta .
$$

Com efeito, para $0<\epsilon \leq \epsilon_{0}, \omega \in H^{1}(\Omega)$ e $\phi \in H^{s}(\Omega)$ e denotando $\Gamma_{\epsilon} \stackrel{\text { def }}{=}\left|\left\langle F_{\epsilon}(u+\omega)-F_{\epsilon}(u)-F_{\epsilon}^{\prime}(u) \omega, \phi\right\rangle\right|$, temos pelo Lema 2.4.1 e aplicando duas vezes o Teorema de Valor Médio que

$$
\begin{aligned}
\Gamma_{\epsilon} & \leq \int_{\Omega}\left|f(u+\omega)-f(u)-f^{\prime}(u) \omega\left\|\phi\left|d x_{2} d x_{1}+\frac{1}{\epsilon^{\alpha}} \int_{o_{\epsilon}}\right| g(u+\omega)-g(u)-g^{\prime}(u) \omega\right\| \phi\right| d x_{2} d x_{1} \\
& \leq\left(\int_{\Omega}\left|f(u+\omega)-f(u)-f^{\prime}(u) \omega\right|^{2} d x_{2} d x_{1}\right)^{1 / 2}\|\phi\|_{L^{2}(\Omega)} \\
& +\left(\frac{1}{\epsilon^{\alpha}} \int_{o_{\epsilon}}\left|g(u+\omega)-g(u)-g^{\prime}(u) \omega\right|^{2} d x_{2} d x_{1}\right)^{1 / 2} C^{1 / 2}\|\phi\|_{H^{s}(\Omega)} \\
& \leq\left(\int_{\Omega}\left|f^{\prime}(u+\theta \omega) \omega-f^{\prime}(u) \omega\right|^{2} d x_{2} d x_{1}\right)^{1 / 2}\|\phi\|_{H^{s}(\Omega)} \\
& +\left(\frac{1}{\epsilon^{\alpha}} \int_{o_{\epsilon}}\left|g^{\prime}(u+\theta \omega) \omega-g^{\prime}(u) \omega\right|^{2} d x_{2} d x_{1}\right)^{1 / 2} C^{1 / 2}\|\phi\|_{H^{s}(\Omega)} \\
& \leq\left(\int_{\Omega}\left|f^{\prime \prime}(u+s \theta \omega) \omega\right|^{2}|\omega|^{2} d x_{2} d x_{1}\right)^{1 / 2}\|\phi\|_{H^{s}(\Omega)}+\left(\frac{1}{\epsilon^{\alpha}} \int_{o_{\epsilon}}\left|g^{\prime \prime}(u+s \theta \omega) \omega\right|^{2}|\omega|^{2} d x_{2} d x_{1}\right)^{1 / 2} C^{1 / 2}\|\phi\|_{H^{s}(\Omega)} \\
& \leq\left\|f^{\prime \prime}\right\|_{L^{\infty}(\mathbb{R})}\left(\int_{\Omega}|\omega|^{4} d x_{2} d x_{1}\right)^{1 / 2}\|\phi\|_{H^{s}(\Omega)}+C^{1 / 2}\left\|g^{\prime \prime}\right\|_{L^{\infty}(\mathbb{R})}\left(\frac{1}{\epsilon^{\alpha}} \int_{o_{\epsilon}}|\omega|^{4} d x_{2} d x_{1}\right)^{1 / 2}\|\phi\|_{H^{s}(\Omega)} \\
& \leq\left\|f^{\prime \prime}\right\|_{L^{\infty}(\mathbb{R})}\|\omega\|_{L^{2}(\Omega)}^{2}\|\phi\|_{H^{s}(\Omega)}+C^{1 / 2}\left\|g^{\prime \prime}\right\|_{L^{\infty}(\mathbb{R})} C^{1 / 2}\|\omega\|_{H^{s}(\Omega)}^{2}\|\phi\|_{H^{s}(\Omega)} \\
& \leq\left(\left\|f^{\prime \prime}\right\|_{L^{\infty}(\mathbb{R})}+C\left\|g^{\prime \prime}\right\|_{L^{\infty}(\mathbb{R})}\right)\|\phi\|_{H^{s}(\Omega)}\|\omega\|_{H^{1}(\Omega)}\|\omega\|_{H^{1}(\Omega)} \cdot
\end{aligned}
$$

Onde $\theta:=\theta\left(x_{1}, x_{2}\right)$ e $s:=s\left(x_{1}, x_{2}\right) \operatorname{com} 0<\theta, s<1$ e $x_{1}, x_{2} \in \bar{\Omega}$. Assim pela definição da norma em $H^{-s}(\Omega)$ temos

$$
\left\|F_{\epsilon}(u+\omega)-F_{\epsilon}(u)-F_{\epsilon}^{\prime}(u) \omega\right\|_{H^{-s}(\Omega)} \leq\left(\left\|f^{\prime \prime}\right\|_{L^{\infty}(\mathbb{R})}+C\left\|g^{\prime \prime}\right\|_{L^{\infty}(\mathbb{R})}\right)\|\omega\|_{H^{1}(\Omega)}\|\omega\|_{H^{1}(\Omega)} .
$$

E como $\delta$ não depende de $\epsilon$ para cada $0<\epsilon \leq \epsilon_{0}$ concluimos que a aplicação $F_{\epsilon}: H^{1}(\Omega) \rightarrow H^{-s}(\Omega)$ é Fréchet diferenciável, uniformemente em $\epsilon$.

Similarmente para $\epsilon=0$ mostremos que para cada $\eta>0$ existe $\delta>0$ tal que

$$
\left\|F_{0}(u+\omega)-F_{0}(u)-F_{0}^{\prime}(u) \omega\right\|_{H^{-s}(0,1)} \leq \eta\|\omega\|_{H^{1}(0,1)}, \quad \omega \in H^{1}(0,1) \quad \text { sempre que } \quad\|\omega\|_{H^{1}(0,1)}<\delta .
$$


Tomando $\phi \in H^{s}(0,1)$ e denotando $\Gamma_{0} \stackrel{\text { def }}{=}\left|\left\langle F_{0}(u+\omega)-F_{0}(u)-\left.F_{0}^{\prime}(u) \omega\right|_{H^{-s}(0,1)}, \phi\right\rangle\right|$, temos

$$
\begin{aligned}
\Gamma_{0} & \leq \int_{0}^{1}\left|f(u+\omega)-f(u)-f^{\prime}(u) \omega\right| p|\phi| d x_{1}+\int_{0}^{1}\left|g(u+\omega)-g(u)-g^{\prime}(u) \omega\right| \mu|\phi| d x_{1} \\
& \leq H_{1}\left(\int_{0}^{1}\left|f^{\prime}(u+\theta \omega) \omega-f^{\prime}(u) \omega\right|^{2} d x_{1}\right)^{1 / 2}\|\phi\|_{L^{2}(0,1)} \\
& +\|\mu\|_{L^{\infty}(0,1)}\left(\int_{0}^{1}\left|g^{\prime}(u+\theta \omega) \omega-g^{\prime}(u) \omega\right|^{2} d x_{1}\right)^{1 / 2}\|\phi\|_{L^{2}(0,1)} \\
& \leq H_{1}\left(\int_{0}^{1}\left|f^{\prime \prime}(u+s \theta \omega)\right|^{2}|\omega|^{4} d x_{1}\right)^{1 / 2}\|\phi\|_{L^{2}(0,1)}+\|\mu\|_{L^{\infty}(0,1)}\left(\int_{0}^{1}\left|g^{\prime \prime}(u+s \theta \omega)\right|^{2}|\omega|^{4} d x_{1}\right)^{1 / 2}\|\phi\|_{L^{2}(0,1)} \\
& \leq\left(H_{1}\left\|f^{\prime \prime}\right\|_{L^{\infty}(\mathbb{R})}+\|\mu\|_{L^{\infty}(0,1)}\left\|g^{\prime \prime}\right\|_{L^{\infty}(\mathbb{R})}\right)\|\omega\|_{H^{1}(0,1)}^{2}\|\phi\|_{H^{s}(0,1)} .
\end{aligned}
$$

Donde,

$$
\left\|F_{0}(u+\omega)-F_{0}(u)-F_{0}^{\prime}(u) \omega\right\|_{H^{-s}(0,1)} \leq\left(H_{1}\left\|f^{\prime \prime}\right\|_{L^{\infty}(\mathbb{R})}+\|\mu\|_{L^{\infty}(0,1)}\left\|g^{\prime \prime}\right\|_{L^{\infty}(\mathbb{R})}\right)\|\omega\|_{H^{1}(0,1)}\|\omega\|_{H^{1}(0,1)}
$$

\subsection{Conjunto de equilíbrios}

Estamos interessados em descrever o conjunto de equilibrios para os problemas (1.7) e (1.5) cujas versões abstratas são definidas em (2.10) e (2.17). Denotamos por soluções de equilibrio (ou pontos de equilibrio) as soluções dos problemas (1.7) e (1.5) e observarmos que tais soluções são respetivamentes as soluções dos problemas abstratos

$$
\begin{array}{ll}
\mathcal{A}_{\epsilon} u_{\epsilon}=F_{\epsilon}\left(u_{\epsilon}\right), & 0<\epsilon \leq \epsilon_{0} \\
\mathcal{A}_{0} u_{0}=F_{0}\left(u_{0}\right), & \epsilon=0 .
\end{array}
$$

Assim o conjunto $\mathcal{E}_{\epsilon}$ introduzido na observação 1.2.6 denotará o conjunto solução dos problemas (3.10) e (3.11) e será definido por

$$
\mathcal{E}_{\epsilon}=\left\{u \in H^{1}(\Omega): \mathcal{A}_{\epsilon} u-F_{\epsilon}(u)=0\right\}, \quad \epsilon \in\left[0, \epsilon_{0}\right]
$$

Definição 3.4.1 Dizemos que a solução $u_{\epsilon}^{*}$ dos problemas (3.10) e (3.11) é hiperbólica se zero não pertence ao espectro do operador $\mathcal{A}_{\epsilon}-F_{\epsilon}^{\prime}\left(u_{\epsilon}^{*}\right)$, isto é, $0 \notin \sigma\left(\mathcal{A}_{\epsilon}-F_{\epsilon}^{\prime}\left(u_{\epsilon}^{*}\right)\right)$.

Proposição 3.4.2 Se u* é uma solução hiperbólica do problema (3.11) (ou (3.10)) então u* é um ponto fixo isolado (isto é, existe uma vizinhança $V \subset H^{1}(0,1)$ (ou $V \subset H^{1}(\Omega)$ ) de $u^{*}$ tal que u é a única solução de $\mathcal{A}_{0} u=F_{0}(u)\left(\right.$ ou $\left.\mathcal{A}_{\epsilon} u=F_{\epsilon}(u)\right)$. 
Demonstração. Temos que $u^{*}$ é um ponto hiperbólico do problema $\mathcal{A l}_{0} u^{*}=F_{0}\left(u^{*}\right)$ se e somente se, a aplicação $\mathcal{A}_{0} u^{*}-F_{0}^{\prime}\left(u^{*}\right): H^{1}(0,1) \rightarrow H^{-s}(0,1)$ for um isomorfismo. Notemos que a aplicação $\Phi\left(u^{*}\right) \stackrel{\text { def }}{=} \mathcal{A}_{0} u^{*}-F_{0}\left(u^{*}\right)$ é Fréchet diferenciável pelo Lema 3.3.2 e pelo fato de $\mathcal{A}_{0} u^{*}$ ser uma aplicação linear. Portanto, segue do Teorema da Aplicação Inversa em espaços de Banach [6] que existe uma vizinhança $V$ de zero em $H^{1}(0,1)$ tal que $\Phi$ é um defeomorfismo.

Por outro lado vimos que $u^{*}$ deve ser ponto fixo da aplicação $\mathcal{A}_{0}^{-1} F_{0}: H^{1}(0,1) \rightarrow H^{1}(0,1)$. Assim $1 \notin \sigma\left(I-\mathcal{A}_{0}^{-1} F_{0}^{\prime}\left(u^{*}\right)\right)$ e como $\mathcal{A}_{0}^{-1} F_{0}$ é compacto pelo Lema 2.2.14 segue pelo Teorema D.2.21 que $u^{*}$ é um ponto fixo isolado e $\left|\operatorname{ind}\left(\mathcal{A}_{0}^{-1} F_{0}, u^{*}\right)\right|=1$.

\subsection{Semi-continuidade inferior}

Finalmente para obter a semi-continuidade no conjunto de equilíbrio $\mathcal{E}_{\epsilon}$ vamos primeiro provar o resultado que nos dá algumas propriedades das aplicações $\mathcal{A}_{\epsilon}^{-1} F_{\epsilon}$. Veremos que a semi-continuidade inferior é uma consequência do [12, Teorema 3].

Lema 3.5.1 Sejam as aplicações $\mathcal{A}_{\epsilon}^{-1} F_{\epsilon}$ definidas em (2.10) e (2.17) para $\epsilon \in[0,1)$. Então:

i) $\mathcal{A}_{\epsilon}^{-1} F_{\epsilon}$ são operadores compactos para cada $\epsilon>0$ fixado.

ii) $\left\{\mathcal{A}_{\epsilon}^{-1} F_{\epsilon}\left(u^{\epsilon}\right)\right\}_{\epsilon \in[0,1)}$ é uma família pré-compacta sempre que $\left\|u^{\epsilon}\right\|_{H_{\epsilon}^{1}(\Omega)}$ é uniformemente limitada, isto é, existe uma subsequência, que ainda denotamos por $\mathcal{A}_{\epsilon}^{-1} F_{\epsilon}\left(u^{\epsilon}\right)$, e $u \in H^{1}(0,1)$ tais que

$$
\left\|\mathcal{A}_{\epsilon}^{-1} F_{\epsilon}\left(u^{\epsilon}\right)-\mathcal{A}_{0}^{-1} F_{0}(u)\right\|_{H_{\epsilon}^{1}(\Omega)} \rightarrow 0, \quad \text { quando } \quad \epsilon \rightarrow 0
$$

iii) Se $\left\|u^{\epsilon}-u\right\|_{H_{\epsilon}^{1}(\Omega)} \rightarrow 0, \quad$ quando $\quad \epsilon \rightarrow 0$, então $\left\|\mathcal{A}_{\epsilon}^{-1} F_{\epsilon}\left(u^{\epsilon}\right)-\mathcal{A}_{0}^{-1} F_{0}(u)\right\|_{H_{\epsilon}^{1}(\Omega)} \rightarrow 0$.

Prova. O ítem $i$ ) segue diretamente pelo Lema 2.2.9 para $0<\epsilon<1$ e Lema 2.2.14 para $\epsilon=0$.

Vamos provar o ítem ii). Seja $u^{\epsilon} \in H_{\epsilon}^{1}(\Omega)$ tais que $\left\|u^{\epsilon}\right\|_{H_{\epsilon}^{1}(\Omega)} \leq C$. Então

$$
\left\|\frac{\partial u^{\epsilon}}{\partial x_{1}}\right\|_{L^{2}(\Omega)}^{2}+\frac{1}{\epsilon^{2}}\left\|\frac{\partial u^{\epsilon}}{\partial x_{2}}\right\|_{L^{2}(\Omega)}^{2}+\left\|u^{\epsilon}\right\|_{L^{2}(\Omega)}^{2} \leq C^{2}
$$

e portanto, temos que

$$
\left\|u^{\epsilon}\right\|_{L^{2}(\Omega),} \quad\left\|\frac{\partial u^{\epsilon}}{\partial x_{1}}\right\|_{L^{2}(\Omega)} \text { e } \frac{1}{\epsilon}\left\|\frac{\partial u^{\epsilon}}{\partial x_{2}}\right\|_{L^{2}(\Omega)} \leq C, \quad \forall \epsilon>0 .
$$

Assim como em (3.3) e (3.4) podemos extrair uma subsequência que denotaremos ainda por $u^{\epsilon}$ tais que

$$
u^{\epsilon} \rightarrow u, w-H^{1}(\Omega), \quad \text { e } \frac{\partial u^{\epsilon}}{\partial x_{2}} \rightarrow 0, s-L^{2}(\Omega)
$$


para algum $u \in H^{1}(0,1)$. Agora consideremos $w^{\epsilon}=\mathcal{A}_{\epsilon}^{-1} F_{\epsilon}\left(u^{\epsilon}\right)$ que é equivalente a $\mathcal{A}_{\epsilon} w^{\epsilon}=F_{\epsilon}\left(u^{\epsilon}\right)$ sendo $w^{\epsilon}$ a solução fraca. Consequentemente

$$
\left\|w^{\epsilon}\right\|_{H_{\epsilon}^{1}(\Omega)}^{2}=\int_{\Omega} f\left(u^{\epsilon}\right) w^{\epsilon} d x_{2} d x_{1}+\frac{1}{\epsilon^{\alpha}} \int_{o_{\epsilon}} g\left(u^{\epsilon}\right) w^{\epsilon} d x_{2} d x_{1}
$$

Como assumimos que as funções $f$ e $g$ são limitadas com derivada limitada Observação 1.2.5 temos pelo Lema 2.4.1 que $w^{\epsilon}$ é uma sequência uniformemente limitada em $H_{\epsilon}^{1}(\Omega)$ para $\epsilon \in(0,1)$. Então podemos argumentar como em (3.3) e (3.4) e em seguida extrair uma subsequência que ainda denotaremos por $w^{\epsilon}$ tal que

$$
w^{\epsilon} \rightarrow w, w-H^{1}(\Omega), \quad \text { e } \quad \frac{\partial w^{\epsilon}}{\partial x_{2}} \rightarrow 0, s-L^{2}(\Omega),
$$

para algum $w \in H^{1}(0,1)$. Assim devido a (3.12) e (3.13) e pelo ítem (3) do Lema 3.3.1 podemos passar o limite em

$$
\left\langle\mathcal{A}_{\epsilon} w^{\epsilon}, \varphi\right\rangle=\left\langle F_{\epsilon}\left(u^{\epsilon}\right), \varphi\right\rangle \quad \text { obtendo }\left\langle\mathcal{A}_{0} w, \varphi\right\rangle=\left\langle F_{0}(u), \varphi\right\rangle,
$$

para cada $\varphi \in H^{1}(0,1)$ e assim $w=\mathcal{A}_{0}^{-1} F_{0}(u)$. No entanto usando de novo o ítem (3) do Lema 3.3.1, obtemos que

$$
\left\|w^{\epsilon}\right\|_{H_{\epsilon}^{1}(\Omega)}=\left\langle F_{\epsilon}\left(u^{\epsilon}\right), w^{\epsilon}\right\rangle \rightarrow\left\langle F_{0}(u), w\right\rangle=\left\langle\mathcal{A}_{0} w, w\right\rangle=\|w\|_{H^{1}(0,1)}
$$

ficando assim provado o ítem ii).

Finalmente, assumindo que $\left\|u^{\epsilon}-u\right\|_{H_{\epsilon}^{1}(\Omega)} \rightarrow 0$ temos que $\left\|u^{\epsilon}\right\|_{H_{\epsilon}^{1}(\Omega)} \leq C$. Assim argumentando como na prova do ítem ii) para qualquer subsequêcia podemos extrair uma outra subsequência tal que

$$
\left\|\mathcal{A}_{\epsilon}^{-1} F_{\epsilon}\left(u^{\epsilon}\right)-\mathcal{A}_{0}^{-1} F_{0}(u)\right\|_{H_{\epsilon}^{1}(\Omega)} \rightarrow 0,
$$

quando $\left\|u^{\epsilon}-u\right\|_{H_{\epsilon}^{1}(\Omega)} \rightarrow 0$. Como mostramos para uma sequência arbitrária obtemos a prova do ítem iii).

Proposição 3.5.2 Seja u uma solução hiperbólica do problema (1.5) satisfazendo $\|u\|_{L^{\infty}(\Omega)} \leq R$ para alguma constante positiva $R$ independente de $\epsilon$. Então existe uma sequência de soluções do problema (1.7) tais que

$$
\left\|u^{\epsilon}-u\right\|_{H^{1}(\Omega)} \rightarrow 0
$$

Demonstração. Temos que $u$ é uma solução hiperbólica do problema (1.5) então pela Proposição 3.4.2 u é uma soluão isolada, isto é, existe $\delta>0$ tal que $u$ é a única solução do problema (1.5) em $B(u, \delta)$, a bola aberta com raio $\delta$ e centro $u \in H^{1}(0,1)$. Além disso $u$ é ponto fixo relativamente a aplicação $\mathcal{A}_{0}^{-1} F_{0}$ satisfazendo $\left|\operatorname{ind}\left(\mathcal{A}_{0}^{-1} F_{0}\right), u\right|=1$ pela Proposição 3.4.2.

Agora como a família de operadores compactos $\mathcal{A}_{\epsilon}^{-1} F_{\epsilon}$ satisfaz os ítem i) e ii) do Lema 3.5.1 segue 
do [12, Teorema 3] que existe $\epsilon_{0}>0 \operatorname{com} \epsilon>\epsilon_{0}$ tal que operador $\mathcal{A}_{\epsilon}^{-1} F_{\epsilon}$ tem pelo menos um ponto fixo $u^{\epsilon} \in B(u, \delta)$ satisfazendo $\left\|u^{\epsilon}-u\right\|_{H_{\epsilon}^{1}(\Omega)}$, quando $\epsilon \rightarrow 0$. Assim completamos a demonstração da semi-continuidade inferior observando que $\|\cdot\|_{H^{1}(\Omega)} \leq\|\cdot\|_{H_{\epsilon}^{1}(\Omega)}$ sempre que $\epsilon \in(0,1)$. 


\section{APÊNDICE A}

\section{CONSIDERAÇÕES GERAIS}

Neste capítulo, fazemos uma breve revisão sobre alguns aspetos relacionados aos espaços $L^{p}$ e suas propriedades. Demonstra-se o Teorema sobre a convergência em $L^{p}(1 \leq p \leq \infty)$ de funções rapidamente oscilantes que será usado ao longo do trabalho. Parte dos resultados foram retirados de $[5,10,13,32,37]$.

\section{A.1 Formas lineares em espaços de Banach}

Definição A.1.1 Sejam X e $Y$ dois espaços de Banach. Definimos o conjunto $\mathcal{L}(X, Y)$ das aplicações lineares e limitadas, isto é

$$
\mathcal{L}(X, Y)=\{\Lambda: X \rightarrow Y \text {, tal que } \Lambda \text { é linear e limitada }\}
$$

munido da norma

$$
\|\Lambda\|_{\mathcal{L}(X, Y)}=\sup _{u \in X \neq\{0\}} \frac{\|\Lambda u\|_{Y}}{\|u\|_{X}} .
$$

Notemos que pela definição da norma $\|\Lambda\|_{\mathcal{L}(X, Y)}$ resulta que para qualquer $u \neq 0$ temos

$$
\frac{\|\Lambda u\|}{\|u\|}=\left\|\Lambda\left(\frac{u}{\|u\|}\right)\right\|
$$

Lema A.1.2 Para todo $u \in X$ tem-se sempre

$$
\|\Lambda u\|_{Y} \leq\|\Lambda\|_{\mathcal{L}(X, Y)}\|u\|_{X}
$$

Prova. Se $u \neq 0$, então o vetor $v=\frac{u}{\|u\|}$ pertence a uma bola unitária logo de (A.1) temos $\|\Lambda\|_{\mathcal{L}(X, Y)}=\sup _{\|u\|_{X} \leq 1}\|\Lambda v\|_{Y}$ implica que

$$
\left\|\Lambda\left(\frac{u}{\|u\|}\right)\right\|=\frac{\|\Lambda u\|_{Y}}{\|u\|_{X}} \leq\|\Lambda\|_{\mathcal{L}(X, Y)}
$$


donde resulta (A.2). Se $u=0$, então ambos membros de (A.2) se anulam.

Definição A.1.3 Uma aplicação linear $\Lambda: X \rightarrow Y$ é limitada se existe uma constante $C>0$ tal que

$$
\|\Lambda u\|_{Y} \leq C\|u\|_{X}, \quad u \in X
$$

O seguinte resultado nos dá a caracterização dos espaços $\mathcal{L}(X, Y)$.

Teorema A.1.4 Seja $\Lambda: X \rightarrow$ Y linear. Então as seguintes afirmações são equivalentes:

i) $\Lambda$ é limitado;

ii) $\Lambda$ é contínuo;

iii) $\Lambda$ é contínuo em um ponto $u_{0} \in X$.

Prova. i) $\Rightarrow$ ii) Seja $\Lambda$ limitado, então da desigualdade (A.2) e pela linearidade de $\Lambda$ segue que

$$
\|\| \Lambda u-\Lambda v\left\|_{Y}=\right\| \Lambda(u-v)\left\|_{Y} \leq\right\| \Lambda\left\|_{\mathcal{L}(X, Y)}\right\| u-v \|_{X}, \quad \forall u, v \in X
$$

donde resulta que $\Lambda$ é contínua, ou seja ii). Que ii) $\Rightarrow$ iii) segue diretamente da continuidade iii) $\Rightarrow$ i) Se $\Lambda$ é contínuo num ponto $u_{0} \in X$, então dado um $\eta>0$, existe um $\delta>0$ tal que

$$
\left\|\Lambda\left(u-u_{0}\right)\right\|_{Y}<\eta \quad \text { sempre que } \quad\left\|u-u_{0}\right\|_{X}<\delta .
$$

Se $v=u-u_{0}$ temos que $\|\Lambda v\|_{Y}<\eta \quad$ sempre que $\|v\|_{X}<\delta$. Consequentemente, fixando um $\omega=\frac{2}{\delta} v$, podemos escrever

$$
\sup _{\|\omega\|_{X}=1}\|\Lambda \omega\|_{Y}=\frac{2}{\delta} \sup _{\|v\|_{X}=\frac{\delta}{2}}\|\Lambda v\|_{Y} \leq \frac{2}{\delta} \eta,
$$

o que implica $i)$, em vertude de (A.2).

Definição A.1.5 Seja X um espaço de Banach. O conjunto das aplicações linerares e contínuas de X sobre $\mathbb{R}$ é chamado espaço dual de $X$ e escreve-se $X^{*}$. Se $x^{*} \in X^{*}$ a imagem $x^{*}(x)$ de $x \in X$ escrever-se-à $\left\langle x^{*}, x\right\rangle_{X^{*}, X} . O$ espaço $X^{* *}=\left(X^{*}\right)^{*}$ de $X^{*}$ chamar-se-á de espaço bidual de $X$.

Em particular o espaço dual $X^{*}=\mathcal{L}(X, \mathbb{R})$ de $X$ é um espaço de Banach munido da norma

$$
\left\|x^{*}\right\|_{X^{*}}=\sup _{x \in X \backslash\{0\}} \frac{\left\|\left\langle x^{*}, x\right\rangle_{X^{*}, X}\right\|}{\|x\|_{X}}, \quad \forall x^{*} \in X^{*} .
$$

No entanto tem lugar a desigualdade (A.2), ou seja,

$$
\left\|\left\langle x^{*}, x\right\rangle_{X^{*}, X}\right\| \leq\left\|x^{*}\right\|_{X^{*}}\|x\|_{X} \quad \forall x \in X .
$$


Pelo que fizemos acima vemos que se $X^{*}$ é um espaço de Banach então o seu espaço dual $X^{* *}$ também é. E podemos iterar e continuar com o processo e assim em particular podemos identificar o espaço $X$ como um subespaço de $X^{* *}$ através de uma isometria canônica. Usaremos a notação seguinte $\langle f, u\rangle$ ou $f u$ para denotar o elemento $f(u)$ onde $f \in X^{*}, u \in X$.

Mostra-se o seguinte resultado sobre a chamada aplicação canônica do espaço X sobre o seu espaço bidual.

Proposição A.1.6 Fixemos $u \in X$ e introduzimos a seguinte aplicação

$$
f u: u^{*} \in X^{*} \mapsto\left\langle u^{*}, u\right\rangle_{X^{*}, X} \in \mathbb{R},
$$

então $f u \in X^{* *}$ e a aplicação

$$
\mathcal{F}: u \in X \mapsto f u \in X^{* *}
$$

é uma isometria, isto é,

$$
\|u\|_{X}=\|\mathcal{F}(u)\|_{X^{* *}}=\|f u\|_{X^{* *}}
$$

Graças a Proposição A.1.6 que conseguimos identificar $u$ com a sua imagem $f u$ e então $X$ com a sua imagem também $\mathcal{F}(X) \subset X^{* *}$.

Definição A.1.7 Seja F a aplicação canônica do espaço X sobre o seu bidual definido na Proposição A.1.6. Então o espaço $X$ é reflexivo se e somente se $\mathcal{F}(X)=X^{* *}$.

Se $X$ é reflexivo devido a propriedade acima podemos identificar o espaço $X$ com seu bidual $X^{* *}$. À seguir, vamos dar uma noção de convergência fraca em um espaço de Banach abstrato $X$ equipado da norma $\|\cdot\|_{X}$. Para tal lembramos inicialmente a definição de convergência forte (ou simplesmente convergência) de uma sequência em $X$.

Definição A.1.8 (Convergência forte) uma sequência $\left\{x_{n}\right\} \in X$ converge fortemente (ou simplesmente converge) para um elemento $x \in X$ e escreve-se $x_{n} \rightarrow x$ (frequentemente iremos usar a seguinte notação $s-X$ para indicar a convergência forte) se e somente se

$$
\left\|x_{n}-x\right\|_{X} \rightarrow 0
$$

Definição A.1.9 i) Sejam $\left\{x_{n}\right\}, x \in X$. Diremos que a sequência $x_{n}$ converge fracamente para $x$ e escrevese $x_{n} \rightarrow x$ fracamente em $X($ ou usando a notação $w-X)$ se

$$
\left\langle x_{n}, x^{*}\right\rangle \rightarrow\left\langle x, x^{*}\right\rangle \quad \forall x^{*} \in X^{*}
$$

ii) Sejam $x_{n}^{*}, x^{*} \in X^{*}$. Diremos que a sequência $x_{n}^{*}$ converge fracamente para $x^{*}$ e escreve-se $x_{n}^{*} \rightarrow x^{*}$ (ou $\left.w^{*}-X^{*}\right)$ fracamente em $X^{*}$ se

$$
\left\langle x, x_{n}^{*}\right\rangle \rightarrow\left\langle x, x^{*}\right\rangle \quad \forall x \in X
$$


Corolário A.1.10 Seja X um espaço de Banach. Então

i) Se $x_{n} \rightarrow x$ (fortemente), implica que $x_{n} \rightarrow x$ (fracamente).

ii) Se $x_{n}^{*} \rightarrow x^{*}$ (fortemente), implica que $x_{n}^{*} \rightarrow x^{*}$ (fracamente $)$.

Prova. Vamos mostrar somente $i$ ) porque ii) mostra-se de modo análogo. Seja $\left\{x_{n}\right\}$ uma sequência em $X$ tal que

$$
x_{n} \rightarrow x \text { fortemente em } X
$$

Então, para todo $x^{*} \in X^{*}$ e utilizando a desigualdade (A.3) segue que

$$
\lim _{n \rightarrow \infty}\left\|\left\langle x_{n}, x^{*}\right\rangle-\left\langle x, x^{*}\right\rangle\right\|=\lim _{n \rightarrow \infty}\left\|\left\langle x_{n}-x, x^{*}\right\rangle\right\| \leq \lim _{n \rightarrow \infty}\left\|x^{*} \mid\right\| x_{n}-x \|=0 .
$$

Teorema A.1.11 (Eberlein-Šmuljan) Seja X um espaço de Banach reflexivo e $\left\{x_{n}\right\}$ uma sequência em X tal que para uma constante positiva K temos que

$$
\left\|x_{n}\right\| \leq K
$$

Então

i) Existe $x \in X$ e uma subsequência $\left\{x_{n j}\right\}$ de $\left\{x_{n}\right\}$ tal que

$$
x_{n j} \rightarrow x \text { fracamente em } X
$$

ii) Se para cada subsequência convergente fracamente de $\left\{x_{n}\right\}$ tem o mesmo limite $x$, então toda sequência $\left\{x_{n}\right\}$ converge fracamente para $x$, isto é

$$
x_{n} \rightarrow x \text { fracamente em } X
$$

\section{A.2 Alguns resultados sobre espaços $L^{P}$}

Seja $U$ é um subconjunto não vazio de $\mathbb{R}^{n}$ e seja $p \in \mathbb{R}$ tal que $1 \leq p \leq \infty$

Definição A.2.1 Para $1 \leq p<+\infty$ temos

$$
L^{p}\left(U, \mathbb{R}^{n}\right)=\left\{f \mid f: U \rightarrow \mathbb{R}, f \text { é mensurável e tal que } \int_{U}|f(x)|^{p} d x<+\infty\right\} .
$$

com a norma em $L^{p}(U, \mathbb{R})$ dada por

$$
\|f\|_{L^{p}\left(U, \mathbb{R}^{n}\right)}=\left(\int_{U}|f(x)|^{p} d x\right)^{\frac{1}{p}} .
$$


Definição A.2.2 Uma função $f: U \rightarrow \mathbb{R}$, mensurável é dita essencialmente limitada se existe $C>0$ tal que $|f(x)|<C$ quase sempre (q.s) em $x \in U$, ou seja

$$
L^{\infty}\left(U, \mathbb{R}^{n}\right)=\{f \mid f: U \rightarrow \mathbb{R}, f \text { é mensurável e tal que existe } C \in \mathbb{R} \operatorname{com}|f(x)| \leq C\}
$$

com a norma em $L^{\infty}\left(U, \mathbb{R}^{n}\right)$ dada por

$$
\|f\|_{L^{\infty}\left(U, \mathbb{R}^{n}\right)}=\operatorname{ess} \sup _{x \in U}|f(x)|=\inf \{C>0 ;|f(x)| \leq C \text { q.s em } x \in U\} .
$$

Notemos que em geral as igualdades (A.4) e (A.5) definem semi-normas uma vez que $\|f\|_{L^{p}\left(U, \mathbb{R}^{n}\right)}=0$ se e somente se, $f \equiv 0$ quase sempre em $U$ (veja [32]). Afim de contornar essa dificuldade define-se em $L^{p}\left(U, \mathbb{R}^{n}\right)$ uma relação $\sim$ dada por

$$
f \sim g \quad \Leftrightarrow \quad f \equiv g \quad \text { quase sempre em } \quad U \text {. }
$$

A relação é uma relação de equivalência.

Assim podemos considerar o quociente $L^{p}\left(U, \mathbb{R}^{n}\right) 1 \leq p \leq \infty$ por $\sim$. As classes de equivalências obtidas por $L^{p}\left(U, \mathbb{R}^{n}\right) / \sim$ formam um espaço vetorial com a norma

$$
\begin{gathered}
\|\{f\}\|_{L^{p}\left(U, \mathbb{R}^{n}\right)}=\left(\int_{U}|f(x)|^{p} d x\right)^{\frac{1}{p}}, \quad 1 \leq p<\infty \\
\|\{f\}\|_{L^{\infty}\left(U, \mathbb{R}^{n}\right)}=\operatorname{ess} \sup _{x \in U}|f(x)|, \quad p=\infty,
\end{gathered}
$$

onde $f$ é a representante de qualquer classe de equivalência $\{f\}$. Por conveniência escreve-se $f \in L^{p}\left(U, \mathbb{R}^{n}\right)$ e $\|f\|_{L^{p}\left(U, \mathbb{R}^{n}\right)}$ para denotar os elementos e a norma em $L^{p}\left(U, \mathbb{R}^{n}\right)$, com $1 \leq p \leq \infty$. Denotaremos $L^{p}\left(U, \mathbb{R}^{n}\right)$ por $L^{p}(U)$ ou simplesmente $L^{p}$ e a $\|f\|_{L^{p}\left(U, \mathbb{R}^{n}\right)}$ por $\|f\|_{L^{p}(U)}$ ou simplesmente $\|f\|_{p} \operatorname{com} 1 \leq p \leq \infty$.

Dizemos que duas funções definem o mesmo elemento de $L^{p}$ quando elas são iguais em quase todo ponto. Mediante esta identificação tem-se

Teorema A.2.3 Para $1 \leq p<\infty L^{p}$ é um espaço de Banach.

Demonstração. veja por exemplo [10, Teorema 6.6].

Se $p=2$ temos $L^{2}(U)$ é o espaço de Hilbert com produto interno dado por

$$
(u, v)_{L^{2}(U)}=\int_{U} u(x) v(x) d x .
$$

Proposição A.2.4 (Desigualdade de Hölder) Seja $1 \leq p<\infty$ e q o seu conjugado isto é,

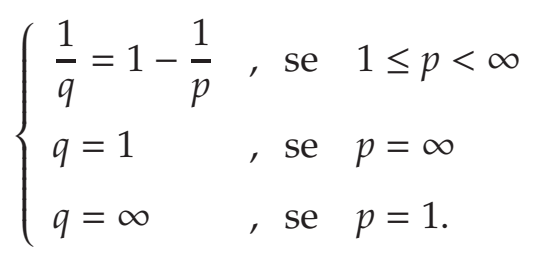


Então para todo $f \in L^{p}(U)$ e $g \in L^{q}(U) \operatorname{com} f g \in L^{1}(U)$ temos:

$$
\int_{U}|f(x) g(x)| d x \leq\|f\|_{L^{p}(U)}\|g\|_{L^{q}(U)}
$$

Demonstração. Veja por exemplo [13, Teorema 4.6].

Para $p=2$ a Desigualdade de Hölder é conhecida como Desigualdade de Cauchy-Schwarz. Uma consequência da Desigualdade de Hölder estabelece como se dão as imersões entre espaços $L^{p}(U)$, quando $U$ tem medida finita.

Corolário A.2.5 Seja $U \subset \mathbb{R}^{n}, \operatorname{com} \mu(U)<+\infty, p$ e q tais que $1 \leq p<q \leq+\infty$. Então

$$
L^{q}(U) \subset L^{p}(U) \quad \text { e } \quad\|\cdot\|_{p} \leq \mu(U)^{1 / p-1 / q}\|\cdot\|_{q} .
$$

Prova. Se $q=\infty$ temos

$$
\|f\|_{p}^{p}=\int_{U}|f| d x \leq\|f\|_{\infty}^{p} \int_{U} 1 d x=\mu(U)\|f\|_{\infty}^{p}
$$

Se $1 \leq p<\infty$, seja $f \in L^{p}(U)$ e apliquemos a desigualdade de Hölder para o par $\left(\frac{p}{q}, \frac{q}{q-p}\right)$. Então

$$
\|f\|_{p}^{p}=\int_{U}|f(x)|^{p}=\int_{U}|f(x)|^{p} \cdot 1 d x \leq\left[\int_{U}|f(x)|^{\frac{p q}{p}} d x\right]^{\frac{p}{q}}\left[\int_{U} 1 d x\right]^{\frac{q-p}{q}}=[\mu(U)]^{\frac{q-p}{q}}\|f\|_{q}^{p} .
$$

Teorema A.2.6 (de representação) Seja $1 \leq p<\infty e \varphi \in\left[L^{p}(U)\right]^{*}$, então existe único $\psi \in L^{q}(U)$ tal que

$$
\langle\varphi, f\rangle=\int_{U} \psi(x) f(x) d x \quad \forall f \in L^{p}(U),
$$

Além disso, verifica-se que:

$$
\|\psi\|_{L^{q}(U)}=\|\varphi\|_{\left[L^{p}(U)\right]^{*}}
$$

Observação A.2.7 Este teorema diz-nos que todo funcional linear contínuo em $L^{p}(U), 1 \leq p<\infty$ pode ser representado por uma integral e a aplicação $\varphi \mapsto \psi$ é uma isometria linear sobrejetivo o que nos permite identificar o espaço abstrato $\left[L^{p}(U)\right]^{*} \operatorname{com} L^{q}(U)$.

Sistematicamente podemos fazer a seguinte identificação $L^{q}(U)=\left[L^{p}(U)\right]^{*}$. Na tabela abaixo encontramse em resumo as principais propriedades de espaços $L^{p}(U)$ quando $U$ é um subconjunto mensurável de $\mathbb{R}^{n}$, nela consideramos $p \neq 1, p \neq 2$ e $q$ é tal que $\frac{1}{p}+\frac{1}{q}=1$. Para mais detalhes veja [13, pág. 103]. 


\begin{tabular}{|l||c|c|c|c|c|}
\hline & Banach & Hilbert & Reflexivo & Separável & Espaço Dual \\
\hline \hline$L^{p}(U)$ & Sim & Não & Sim & Sim & $L^{q}(U)$ \\
\hline$L^{1}(U)$ & Sim & Não & Não & Sim & $L^{\infty}(U)$ \\
\hline$L^{2}(U)$ & Sim & Sim & Sim & Sim & $L^{2}(U)$ \\
\hline$L^{\infty}(U)$ & Sim & Não & Não & Não & Contém $L^{1}(U)$ \\
\hline
\end{tabular}

Definição A.2.8 Seja A um conjunto mensurável em $\mathbb{R}^{n}$, a função característica de A é a função $\chi_{A}$ definida por

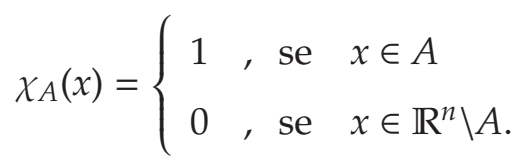

Definição A.2.9 A função $f: \mathbb{R}^{n} \rightarrow \mathbb{R}$ chama-se função simples se

$$
f(x)=\sum_{k=1}^{n} \alpha_{k} \chi_{I_{k}}
$$

com $m \in \mathbb{N}, \alpha_{k} \in \mathbb{R} e I_{k}=\prod_{k=1}^{n}\left[a_{k}, b_{k}\right]$ é um intervalo em $\mathbb{R}^{n} \forall k=\{1,2, \ldots, m\}$.

Se $U \subset \mathbb{R}^{n}$ é um conjunto aberto e limitado, denotamos por $S(U)$ o conjunto de todas funções simples da forma $\sum_{k=1}^{m} \alpha_{k} \chi_{I_{k}}$. Fato $S(U) \subset L^{p}(U), \forall p$ tal que $1 \leq p<\infty$.

Proposição A.2.10 Para $1 \leq p<\infty$, então $S(U) \operatorname{com} \mu(U)<\infty$, é denso em $L^{p}(U)$.

Demonstração. veja por exemplo [10, Proposição 6.7].

Observação A.2.11 Segue deste Proposição A.2.10 em particular que $L^{p}(U)$ é denso em $L^{1}(U)$.

Teorema A.2.12 Para $1 \leq p<\infty$ e um aberto $U$. O conjunto das funções contínuas em $U$ é denso em $L^{p}(U)$.

Demonstração. Veja por exemplo [37, Teorema 3.14].

Definição A.2.13 (Função Localmente Integrável) Sejam $1 \leq p<\infty$ e U um subconjunto aberto de $\mathbb{R}^{n}$. A função $f: U \rightarrow \mathbb{R}$ é localmente integrável em $L^{p}(U)$ e denotaremos $L_{\text {loc }}^{p}(U)$ se for mensurável e para qualquer compacto $\mathrm{K}$ tal que $\mathrm{K} \subset \mathrm{U}$ temos

$$
\int_{K}|f(x)|^{p} d x<\infty
$$

Proposição A.2.14 Seja $1 \leq p \leq \infty$. Então $L^{p}(U) \subset L_{l o c}^{1}(U)$ e $\|\cdot\|_{1} \leq C\|\cdot\|_{p}$.

Demonstração. Sem perda de generalidade suponhamos que $f \in L_{l o c}^{p}(U), 1<p<\infty$. Tomemos um 
subconjunto compacto $K$ de $U$. Seja $\chi_{K}$ a função característica de $K$, temos que $\chi_{K} \in L_{l o c}^{q}(U)$ para qualquer que seja $q$. Aplicando a desiguladade de Hölder temos:

$$
\int_{K}|f(x)| d x=\int_{U}|f(x)| \chi_{K}(x) d x \leq\left(\int_{K}|f(x)|^{p}\right)^{\frac{1}{p}}\left(\int_{K} d x\right)^{\frac{1}{q}}=\|f\|_{p}|K|^{q^{-1}} .
$$

Tomando $C=|K|^{q^{-1}}$, onde $q$ é o expoente conjugado de $p$, chegamos ao resultado.

\section{A.3 Convergência fraca em $L^{p}$}

Seja $U$ um subconjunto aberto limitado e suave de $\mathbb{R}^{n}$ e $p, q$ conjugados (veja A.6).

Definição A.3.1 Uma sequência $\left\{u_{n}\right\}$ em $L^{p}(U)$ converge fracamente para $u \in L^{p}(U)$, e neste caso escrevemos

$$
u_{n} \rightarrow u \text { fracamente em } L^{p}(U)
$$

se

$$
\int_{U} u_{n} v d x \rightarrow \int_{U} u v d x, \quad \forall v \in L^{q}(U)
$$

Se $p=\infty$, dizemos que a sequência $\left\{u_{n}\right\}$ converge fracamente* para $u \in L^{\infty}(U)$ e nesse caso escrevemos

$$
u_{n} \rightarrow u \text { fracamente } e^{*} \quad L^{\infty}(U)
$$

se

$$
\int_{U} u_{n} v d x \rightarrow \int_{U} u v d x, \quad \forall v \in L^{1}(U)
$$

Teorema A.3.2 (sobre a limitação duma sequência convergente fracamente) Suponhamos que a sequência $1 \leq p<\infty$ e $u_{n} \rightarrow$ u fracamente em $L^{p}(U)\left(\rightarrow\right.$ fracamente $^{*}$ em $L^{\infty}(U)$ se $\left.p=\infty\right)$. Então

(a) A sequência $\left\{u_{n}\right\}$ é limitada em $L^{p}(U)$, isto é, existe uma contante $C>0$ independente de $n$ tal que

$$
\forall n \in \mathbb{N} \quad\left\|u_{n}\right\|_{L^{p}(U)} \leq C
$$

(b) A norma é semi-contínua inferior em relação a convergência fraca, isto é,

$$
\|u\|_{L^{p}(U)} \leq \lim _{n \rightarrow \infty} \inf \left\|u_{n}\right\|_{L^{p}(U)}
$$

Teorema A.3.3 (sobre a convergência fraca em $L^{p}$ ) Suponha $1<p<\infty$ e a sequência $\left\{u_{n}\right\}$ é limitada em $L^{p}(U)$. Então existe uma subsequência que ainda denotaremos por $\left\{u_{n}\right\}$ e uma função $u \in L^{p}(U)$ tal que

$$
u_{n} \rightarrow u, \text { fracamente em } L^{p}(U)
$$

Se $p=\infty$, o resultado ainda vale substituindo fracamente por fracamente*. 
Observação A.3.4 O Teorema A.3.3 é falso quando $p=1$ uma vez que $L^{1}(U)$ não é dual de $L^{\infty}(U)$. Mas existe um resultado que substitue $L^{1}(U)$ como um subconjunto de um espaço chamado Espaço de Medida de Radon que denotemos por $\mathcal{M}(U)$ e usa a topologia fraca*. E define-se como sendo o espaço dual do espaço das funções contínuas com suporte compacto $C_{c}(U)$, isto é, $\left[C_{c}(U]^{\prime}=\mathcal{M}(U)\right.$, para mais detalhes veja por exemplo [5, pág, 23].

Teorema A.3.5 Seja $1<p<\infty$ e $\left\{u_{n}\right\}$ uma sequência em $L^{p}(U)$. Então as seguintes afirmações são equivalentes:

(1) $u_{n} \rightarrow u$ fracamente em $L^{p}(U) \Longleftrightarrow$

$$
\begin{cases}\text { (i) } \quad\left\|u_{n}\right\|_{L^{p}(U)} \leq C & \text { (independente de } n \text { ) } \\ \text { (ii) } \quad \int_{I} u_{n} d x \rightarrow \int_{I} u d x, & \text { para todo aberto } I \subset U .\end{cases}
$$

Se $p=\infty$, o resultado ainda continua válido substituindo fracamente por fracamente* veja por exemplo [5, pág. 25].

Demonstração. Suponhamos que vale (1) pelo Teorema A.3.2 (a) segue (i). Agora aplicando a definição de convergência fraca para a função $\varphi=\chi_{I}$ segue (ii). O que implica que (1) $\Rightarrow(2)$.

(2) $\Rightarrow(1)$ Seja $\varphi \in L^{q}(U) \operatorname{com} \frac{1}{p}+\frac{1}{q}=1$ pelo Teorma A.2.10 para todo positivo $\eta$ existe uma função simples $\varphi_{\eta}$ tal que:

$$
\left\|\varphi-\varphi_{\eta}\right\|_{L^{q}(U)} \leq \eta
$$

com

$$
\varphi_{\eta}=\sum_{k=1}^{m} \alpha_{k} \chi_{I_{k}}
$$

onde $m \in \mathbb{N}, \alpha_{k} \in \mathbb{R}$ e $I_{k}=\prod_{k=1}^{n}\left[a_{k}, b_{k}\right]$ é um intervalo aberto em $U$ para todo $k \in\{1,2, \ldots, m\}$. Então

$$
\int_{U}\left(u_{n}-u\right) \varphi d x=\int_{U}\left(u_{n}-u\right)\left(\varphi_{\eta}+\varphi-\varphi_{\eta}\right) d x=\int_{U}\left(u_{n}-u\right) \varphi_{\eta} d x+\int_{U}\left(u_{n}-u\right)\left(\varphi-\varphi_{\eta}\right) d x
$$

Em (ii) temos que quando $n \rightarrow \infty$

$$
\int_{U}\left(u_{n}-u\right) \varphi_{\eta}=\sum_{k=1}^{m} \alpha_{k} \int_{I_{k}}\left(u_{n}-u\right) d x \rightarrow 0
$$

Em (i) aplicando a desigualdade de Hölder temos que

$$
\int_{U}\left(u_{n}-u\right)\left(\varphi-\varphi_{\eta}\right) d x \leq \int_{U}\left|\left(u_{n}-u\right)\left(\varphi-\varphi_{\eta}\right)\right| d x \leq\left\|u_{n}-u\right\|_{L^{p}(U)}\left\|\varphi-\varphi_{\eta}\right\|_{L^{q}(U)} \leq C_{1} \eta,
$$

onde $C_{1}$ é independente de $n$ e $\eta$. Então 1 ) segue de (A.7) fazendo primeiro $n \rightarrow \infty$ e então $\eta \rightarrow 0$. 
Observação A.3.6 Notemos que os Teoremas A.3.2, A.3.3 e A.3.5 dão-nos uma caracterização da convergência fraca em espaços $L^{p}$ mas podem ser enunciados para um contexto mais geral, ou seja para qualquer espaço de Banach abstrato X.

\section{A.4 Funções periódicas rapidamente oscilantes}

Nesta seção vamos estudar uma importante classe de funções rapidamente oscilantes da forma

$$
a_{\epsilon}(x)=a\left(\frac{x}{\epsilon}\right)
$$

onde $a$ é uma função periódica e $\epsilon$ é um parametro positivo que toma seus valores em sequências tendentes a zero.

\section{A.4.1 Funções periódicas em $L^{1}$}

Denotemos por $Y$ um hipercubo em $\mathbb{R}^{n}$ definido por:

$$
Y=] 0, l_{1}[\times] 0, l_{2}[\times \ldots, \times] 0, l_{n}[,
$$

onde $l_{1}, l_{2}, \ldots, l_{n}$ são números inteiros positivos. Referiremo-nos a $Y$ como período referencial.

Definição A.4.1 Seja $Y$ definido em (A.8) e $f$ uma função definida quase sempre em $\mathbb{R}^{n}$. A função $f$ é $Y$ periódica se e somente se

$$
f\left(x+k l_{i} e_{i}\right)=f(x), \text { q.s em } \mathbb{R}^{n}, \forall k \in \mathbb{Z}, \forall i \in\{1,2, \ldots, n\},
$$

onde $\left\{e_{1}, e_{2}, \ldots, e_{n}\right\}$ é uma base de $\mathbb{R}^{n}$.

No caso $n=1$ dizemos simplesmente que $f$ é $l_{1}$-periódico. Notemos que se $f$ é uma função $Y$ periódica, então $f_{\epsilon}$ é uma função $\epsilon Y$ - periódica.

Definição A.4.2 Seja U um conjunto aberto e limitado de $\mathbb{R}^{n}$ e uma função $f \in L^{1}(U)$. O valor médio de $f$ sobre U é um número real $\mathcal{M}_{U}(f)$ dado por

$$
\mathcal{M}_{U}(f)=\frac{1}{|U|} \int_{U} f(y) d y
$$

Agora vamos provar um resultado que diz que o valor médio de uma função periódica qualquer pode ser calculado em qualquer conjunto transladado do período de referência. 
Lema A.4.3 Seja $f$ uma função $Y$ - periódica em $L^{1}(Y)$. Seja $y_{0}$ um ponto fixado em $\mathbb{R}^{n}$ e denotemos por $Y_{0} o$ conjunto transladado de $Y$ dado por

$$
Y_{0}=y_{0}+Y
$$

Seja

$$
f_{\epsilon}(x)=f\left(\frac{x}{\epsilon}\right) \quad \text { q.sem } \mathbb{R}^{n}
$$

então

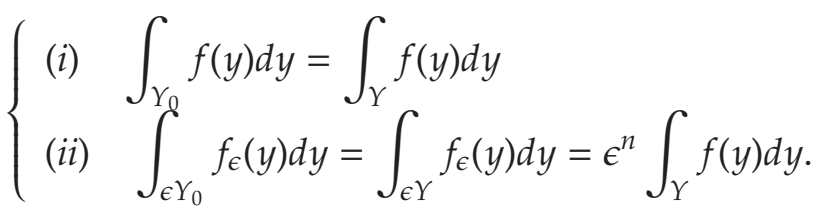

Prova. Se $y_{0}=\left(y_{0}^{1}, y_{0}^{2}, \ldots, y_{0}^{n}\right)$. Então $\left.Y_{0}=\right] y_{0}^{1}, y_{0}^{1}+l_{1}[\times] y_{0}^{2}, y_{0}^{2}+l_{2}[\times \ldots, \times] y_{0}^{n}, y_{0}^{n}+l_{n}[$. Seja $i \in\{1,2, \ldots, n\}$ fixado e a função

$$
\Phi\left(y_{i}\right)=\int_{\prod_{j \neq i} y_{0^{j}}^{j} y_{0}^{j}+l_{j}[} f d y_{1} d y_{2} \ldots d y_{i-1} d y_{i+1} \ldots d y_{n}
$$

é uma função $l_{i}$ - periódica. Então para cada um dos períodos tem se

$$
\begin{aligned}
\int_{Y_{0}} f(y) d y & =\int_{y_{0}^{1}}^{y_{0}^{1}+l_{1}} \Phi\left(y_{1}\right) d y_{1}=\int_{y_{0}^{1}}^{0} \Phi\left(y_{1}\right) d y_{1}+\int_{0}^{y_{0}^{1}+l_{1}} \Phi\left(y_{1}\right) d y_{1} \\
& =-\int_{0}^{y_{0}^{1}} \Phi\left(y_{1}\right) d y_{1}+\int_{0}^{l_{1}} \Phi\left(y_{1}\right) d y_{1}+\int_{l_{1}}^{y_{0}^{1}+l_{1}} \Phi\left(y_{1}\right) d y_{1} \\
& =-\int_{0}^{y_{0}^{1}} \Phi\left(y_{1}\right) d y_{1}+\int_{0}^{y_{0}^{1}} \Phi\left(y_{1}+l_{1}-l_{1}\right) d y_{1}+\int_{0}^{l_{1}} \Phi\left(y_{1}\right) d y_{1} \\
& =\int_{0}^{l_{1}} \Phi\left(y_{1}\right) d y_{1}=\int_{0}^{l_{1}} \int_{\prod_{j \neq 1} y_{0}^{j}, y_{0}^{j}+l_{j}[} f d y_{2} d y_{3} \ldots d y_{n} .
\end{aligned}
$$

Notemos que

$$
\int_{\prod_{j \neq 1} y_{0}^{j}, y_{0}^{j}+l_{j}[} f d y_{2} d y_{3} \ldots d y_{n}=\int_{y_{0}^{2}}^{y_{0}^{2}+l_{2}} \int_{\prod_{j>2} y_{0}^{j}, y_{0}^{j}+l_{j}[} f d y_{2} d y_{3} \ldots d y_{n}=\int_{y_{0}^{2}}^{y_{0}^{2}+l_{2}} \Phi\left(y_{2}\right) d y_{2} .
$$

Repitindo um cálculo similar na direção de $y_{2}$ obtemos

$$
\int_{\left.\prod_{j \neq 1}\right] y_{0}^{j}, y_{0}^{j}+l_{j}[} f d y_{2} d y_{3} \ldots d y_{n}=\int_{0}^{l_{2}} \int_{\left.\prod_{j>2}\right] y_{0}^{j}, y_{0}^{j}+l_{j}[} f d y_{2} d y_{3} \ldots d y_{n}
$$

portanto,

$$
\int_{Y_{0}} f d y=\int_{0}^{l_{1}} \int_{0}^{l_{2}} \int_{\prod_{j>2} y_{0}^{j}, y_{0}^{j}+l_{j}[} f d y_{2} d y_{3} \ldots d y_{n}
$$


Então (i) segue repitindo sucessivamente o mesmo argumento para $y_{3}, y_{4}, \ldots, y_{n}$. Por mudança de variável e um cálulo similar a (i) obtemos (ii), o que completa a prova do lema.

Para concluir este capítulo vamos provar o resultado sobre a convergência fraca em espaços $L^{p}$ de funções periódicas que é uma generalização ao fato de que uma função periódica converge fracamente para seu valor médio quando o número de oscilações aumenta.

Teorema A.4.4 Seja $1 \leq p \leq \infty$ e f uma função $Y$-periódica em $L^{p}(Y)$. Seja $f_{\epsilon} \in L^{p}(U)$ definido por

$$
f_{\epsilon}(x)=f\left(\frac{x}{\epsilon}\right) . \quad \text { q.s em } \mathbb{R}^{n},
$$

onde U é um subconjunto aberto e limitado de $\mathbb{R}^{n}$.

Então, se $1 \leq p<\infty$,

$$
f_{\epsilon} \rightarrow \mathcal{M}_{Y}(f)=\frac{1}{|Y|} \int_{Y} f(y) d y, \quad \text { fracamente em } L^{p}(U), \quad \text { quando } \quad \epsilon \rightarrow 0 .
$$

Se $p=\infty$,

$$
f_{\epsilon} \rightarrow \mathcal{M}_{Y}(f)=\frac{1}{|Y|} \int_{Y} f(y) d y, \quad \text { fracamente }{ }^{*} \text { em } L^{\infty}\left(\mathbb{R}^{n}\right), \quad \text { quando } \quad \epsilon \rightarrow 0 .
$$

Demonstração. Para provar este teorema vamos mostrar que se $1 \leq p \leq \infty$, então

1) $\left\|f_{\epsilon}\right\|_{L^{p}(U)} \leq C$, ou seja que a sequência $\left\{f_{\epsilon}\right\}$ é limitada em $L^{p}(U)$ para todo subconjunto aberto e limitado de $\mathbb{R}^{n}$, onde $C$ é uma constante positiva independente de $\epsilon$.

2) $\lim _{\epsilon \rightarrow 0} \int_{I}\left(f_{\epsilon}(x)-\mathcal{M}_{Y}(f)\right) d x=0$ para todo hipercubo $I$ de $\mathbb{R}^{n}$

Para tal vamos decompor em etapas a saber:

\section{Etapa 1. Estimativa priori}

Se $p=\infty$, então $f \in L^{\infty}(Y)$ e $f$ é $Y$-periódico e como $f_{\epsilon}$ é definido em (A.10) podemos deduzir que

$$
\left\|f_{\epsilon}\right\|_{L^{\infty}\left(\mathbb{R}^{n}\right)}=\|f\|_{L^{\infty}(Y)}
$$

Consequentemente pelo Teorema A.3.3 existe uma subsequência $\left\{f_{\epsilon^{\prime}}\right\}$ tal que

$$
f_{\epsilon^{\prime}} \rightarrow F \quad \text { fracamente* em } \quad L^{\infty}\left(\mathbb{R}^{n}\right)
$$

Agora consideremos $1 \leq p<\infty$. Seja $U$ subconjunto aberto e limitado de $\mathbb{R}^{n}$, escolhemos $\epsilon_{0} \in \mathbb{N}$ tal que $\epsilon_{0} U \supset Y$. Então pelo Lema A.4.3 temos

$$
\begin{aligned}
\left\|f_{\epsilon}\right\|_{L^{p}(Y)}^{p} & =\int_{Y}\left|f\left(\frac{x}{\epsilon}\right)\right|^{p} d x=\epsilon^{n} \int_{\epsilon^{-1} Y}|f(x)|^{p} d x \\
& \leq \epsilon^{n} \int_{\epsilon^{-1} \epsilon_{0} U}|f(x)|^{p} d x=\epsilon^{n} \epsilon^{-n} \epsilon_{0}^{n} \int_{U}|f(x)|^{p} d x=\epsilon_{0}^{n}\|f\|_{L^{p}(U)}^{p} .
\end{aligned}
$$


Para obter a estimativa priori em $L^{p}(U)$ é suficiente mostrar que para todo hipercubo $I$ de $\mathbb{R}^{n}$, existe uma constante $C>0$ independente de $\epsilon$ tal que

$$
\left\|f_{\epsilon}\right\|_{L^{p}(I)} \leq C
$$

Podemos supor que $I$ contém no mínimo um conjunto transladado de $Y$.

Notemos que podemos obter $n(\epsilon)$ conjuntos disjuntos de $Y$ denotado por $Y_{k}, k=1,2, \ldots, n(\epsilon)$ com $\epsilon Y_{k} \subset I$ e $n^{\prime}(\epsilon)$ conjuntos disjuntos transladados de $Y$ denotados por $Y_{j}^{\prime}, j=1,2, \ldots, n^{\prime}(\epsilon)$ com $\epsilon Y_{j}^{\prime} \cap \partial I \neq \emptyset$, tal que

$$
I \subset\left(\bigcup_{k=1}^{n(\epsilon)} \epsilon \overline{Y_{k}}\right) \cup\left(\bigcup_{j=1}^{n^{\prime}(\epsilon)} \epsilon \overline{Y_{j}^{\prime}}\right)
$$

Sejam $L_{1}, L_{2}, \ldots, L_{n}$ os comprimentos das arestas de $I$. mostremos primeiro que

$$
\left\{\begin{array}{l}
\text { (i) } \lim _{\epsilon \rightarrow 0} \epsilon^{n} n(\epsilon)=\frac{|I|}{|Y|} \\
\text { (ii) } \lim _{\epsilon \rightarrow 0} \epsilon^{n-1} n^{\prime}(\epsilon) \leq \sum_{i=1}^{n}\left(\prod_{j \neq i} \frac{L_{j}}{l_{j}}\right) \leq n \frac{|I|}{|Y|} .
\end{array}\right.
$$

Para cada $\epsilon>0$, existe $k_{1}^{\epsilon}, k_{2}^{\epsilon}, \ldots, k_{n}^{\epsilon} \in \mathbb{N}$ tais que para todo $i=1,2, \ldots, n$ tem se

$$
L_{i}=\epsilon k_{i}^{\epsilon} l_{i}+\gamma_{i}^{\epsilon}, \quad \text { com } 0 \leq \gamma_{i}^{\epsilon} \leq \epsilon l_{i}
$$

e consequentemente

$$
\epsilon k_{i}^{\epsilon} \rightarrow \frac{L_{i}}{l_{i}}, \quad \text { quando } \epsilon \rightarrow 0 .
$$

Por outro lado observemos que o número de períodos transladados de $\epsilon$ estritamente incluidos em $I$ é

$$
n_{\epsilon}=k_{1}^{\epsilon} \times k_{2}^{\epsilon} \times \cdots \times k_{n}^{\epsilon}
$$

Assim de (A.14) implica que

$$
\epsilon^{n} n_{\epsilon} \rightarrow \frac{L_{1} \times L_{2} \times \cdots \times L_{n}}{l_{1} \times l_{2} \times \cdots \times l_{n}}=\frac{|I|}{|Y|}
$$

que é a afirmação (A.13)(i).

Para avaliar $n_{\epsilon}^{\prime}$, observemos que os conjuntos $Y_{k}$ e $Y_{j}^{\prime}$ podem ser escolhidos de tal modo que o hipercubo $I$ seja coberto pela união disjunta dos conjuntos $K^{\epsilon}$ transladados de $\epsilon \bar{Y}$ definidos por $K^{\epsilon}=\left(k_{1}^{\epsilon}+1\right) \times\left(k_{2}^{\epsilon}+1\right) \times \cdots \times\left(k_{n}^{\epsilon}+1\right)$, donde

$$
K^{\epsilon}=\prod_{i=1}^{n} k_{i}^{\epsilon}+\sum_{i=1}^{n}\left(\prod_{j \neq i} k_{j}^{\epsilon}\right)+\sum_{i=1}^{n} k_{i}^{\epsilon}+1 .
$$

Então

$$
n_{\epsilon}^{\prime} \leq K^{\epsilon}-n_{\epsilon}=A_{\epsilon}+B_{\epsilon}
$$




$$
A_{\epsilon}=\sum_{i=1}^{n}\left(\prod_{j \neq i} k_{j}^{\epsilon}\right) \text { e } B_{\epsilon}=\sum_{i=1}^{n} k_{i}^{\epsilon}+1 .
$$

Assim de (A.14), vemos que

$$
\epsilon^{n-1} A_{\epsilon}=\sum_{i=1}^{n}\left(\prod_{j \neq i} \epsilon^{n-1} k_{j}^{\epsilon}\right) \rightarrow \sum_{i=1}^{n}\left(\prod_{j \neq i} \frac{L_{j}}{l_{j}}\right) \leq n \prod_{j=1}^{n} \frac{L_{j}}{l_{j}}=n \frac{|I|}{|Y|^{\prime}}
$$

para todo $j=1,2, \ldots, n$ tem-se $l_{j}<L_{j}$. Isto implica (A.13)(ii) uma vez que pela construção os $B_{\epsilon}$ são de ordem $n-2$ em $k_{i}^{\epsilon}$ multiplicando por $\epsilon^{n-1}$ obtemos uma soma finita de de $n$ termos que convergem para zero, ou seja,

$$
\epsilon^{n-1} B_{\epsilon}=\sum_{i=1}^{n} \epsilon^{n-1} k_{i}^{\epsilon}+\epsilon^{n-1} \rightarrow 0, \quad \text { quando } \quad \epsilon \rightarrow 0 \quad(n>1) .
$$

Agora a periodicidade de $f$, e o Lema A.4.3 dão-nos que

$$
\begin{aligned}
\left\|f_{\epsilon}\right\|_{L^{p}(I)}^{p} & \leq \sum_{k=1}^{n(\epsilon)} \int_{\epsilon Y_{k}}\left|f_{\epsilon}\right|^{p} d x+\sum_{j=1}^{n^{\prime}(\epsilon)} \int_{\epsilon Y_{j}^{\prime}}\left|f_{\epsilon}\right|^{p} d x=\sum_{k=1}^{n(\epsilon)} \epsilon^{n} \int_{Y_{k}}|f(x)|^{p} d x+\sum_{j=1}^{n^{\prime}(\epsilon)} \epsilon^{n} \int_{Y_{j}^{\prime}}|f(x)| d x \\
& =\left(\sum_{k=1}^{n(\epsilon)} 1+\sum_{j=1}^{n^{\prime}(\epsilon)} 1\right) \epsilon^{n} \int_{Y}|f(x)|^{p} d x=\left[n(\epsilon)+n^{\prime}(\epsilon)\right] \epsilon^{n} \int_{Y}|f(y)|^{p} d y \\
& \leq C\|f\|_{L^{p}(Y)}^{p} .
\end{aligned}
$$

É claro que $C$ é uma constante arbitrária positiva e independente de $\epsilon$, pois pela estimativa (A.13) temos que:

$$
\left[n(\epsilon)+n^{\prime}(\epsilon)\right] \epsilon^{n}=n(\epsilon) \epsilon^{n}+\epsilon n^{\prime}(\epsilon) \epsilon^{n-1} \rightarrow \frac{|I|}{|Y|}+0 \cdot n \frac{|I|}{|Y|}=\frac{|I|}{|Y|}, \quad \text { quando } \quad \epsilon \rightarrow 0 .
$$

O que significa que a sequência $\left\{f_{\epsilon}\right\}$ é limitada em $L^{p}(U)$ para qualquer subconjunto aberto $U$ de $\mathbb{R}^{n}$. Em particular para $1<p<\infty$ assim podemos aplicar o Teorema A.3.3 para extrair uma subsequência $\left\{f_{\epsilon^{\prime}}\right\}$ tal que

$$
f_{\epsilon^{\prime}} \rightarrow G \text { fracamente em } L^{p}(U)
$$

\section{Etapa 2. Identificação do limite}

(a) Caso $1<p<\infty$

Seja $U$ um subconjunto aberto de $\mathbb{R}^{n}$. Pela primeira etapa e o Teorema A.3.5 para identificar o limite em (A.16), é suficiente mostrar que

$$
\int_{I} f_{\epsilon}(x) \varphi d x \rightarrow \int_{I} \mathcal{M}_{Y}(f) \varphi=|I| \mathcal{M}_{Y}(f)=\frac{|I|}{|Y|} \int_{Y} f(x) d x
$$


$\forall \varphi \in L^{1}(U)$ e para todo hipercubo $I \subset U$. Utilizando (A.12), $\varphi=1$ e pela Observação A.2.11 tem-se

$$
\begin{aligned}
\int_{I} f_{\epsilon}(x) d x & =\sum_{k=1}^{n(\epsilon)} \int_{\epsilon Y_{k}} f_{\epsilon}(x) d x+\sum_{j=1}^{n^{\prime}(\epsilon)} \int_{\epsilon Y_{j}^{\prime} \cap I} f_{\epsilon}(x) d x \\
& =n(\epsilon) \epsilon^{n} \int_{Y} f(y) d y+\sum_{j=1}^{n^{\prime}(\epsilon)} \int_{\epsilon Y_{j}^{\prime} \cap I} f_{\epsilon}(x) d x .
\end{aligned}
$$

Da estimativa (A.13) segue que

$$
n(\epsilon) \epsilon^{n} \int_{Y} f(y) d y \rightarrow \frac{|I|}{|Y|} \int_{Y} f(y) d y=|I| \mathcal{M}_{Y}(f)=\int_{I} \mathcal{M}_{Y}(f) d x
$$

e também

$$
\left|\sum_{j=1}^{n^{\prime}(\epsilon)} \int_{\epsilon Y_{j}^{\prime} \cap I} f_{\epsilon}(x) d x\right| \leq n^{\prime}(\epsilon)\left|\int_{\epsilon Y} f_{\epsilon}(x) d x\right|=\epsilon^{n} n^{\prime}(\epsilon)\left|\int_{Y} f(y) d y\right| \rightarrow 0 .
$$

Consequentemente, em (A.16) tem-se $G=\mathcal{M}_{Y}(f)$. No entanto pelo ii) Teorema A.1.11 implica que qualquer sequência de $\left\{f_{\epsilon}\right\}$ converge para $\mathcal{M}_{Y}(f)$.

(b) Caso $p=\infty$

Seja $U$ um subconjunto aberto limitado de $\mathbb{R}^{n}$ e $\chi_{U}$ uma função característica. Em particular para toda $\phi \in L^{2}(U)$ tem-se $\phi \chi_{U} \in L^{1}(U)$. Então de (A.11) obtemos que

$$
\int_{U} f_{\epsilon^{\prime}} \phi d x \rightarrow \int_{U} F \phi d x
$$

desde que

$$
f_{\epsilon^{\prime}} \rightarrow F \quad \text { fracamente em } \quad L^{2}(U)
$$

Pelo caso anterior e pela unicidade de limite temos que

$$
F=\mathcal{M}_{Y}(f) \text { q.s em } U
$$

e por conseguinte quase sempre em $\mathbb{R}^{n}$, uma vez que $U$ é arbitrário. De novo pelo ii) Teorema A.1.11 implica que qualquer sequência de $\left\{f_{\epsilon}\right\}$ converge para $\mathcal{M}_{Y}(f)$.

(c) Caso $p=1$

Como o conjunto limitado no espaço $L^{1}$ não é fracamente compacto então não podemos aplicar o argumento usado nos casos anteriores. Para provar o resultado vamos aplicar o argumento de densidade. Com efeito para todo $\eta>0$, existe $g \in L^{2}(Y)$ tal que

$$
\|f-g\|_{L^{1}(Y)} \leq \eta
$$


Vamos estendemos $g$ por periodicidade em quase todo ponto de $\mathbb{R}^{n}$ por

$$
g\left(x+k l_{i} e_{i}\right)=g(x) \quad \text { q.s } \quad \text { em } \quad Y \quad \forall k \in \mathbb{Z} \quad \forall i \in\{1,2, \ldots, n\}
$$

onde $\left\{e_{1}, e_{2}, \ldots, e_{n}\right\}$ é a base canónica de $\mathbb{R}^{n}$. Defina $g_{\epsilon}(x)$ por

$$
g_{\epsilon}(x)=g\left(\frac{x}{\epsilon}\right) \quad \text { em } \quad \mathbb{R}^{n}
$$

Seja $U$ um subconjunto aberto e limitado de $\mathbb{R}^{n}$. Então para todo $\varphi \in L^{\infty}(U)$, e adicionando e subtraindo termos correspondentes tem-se

$$
\begin{aligned}
\int_{U}\left(f_{\epsilon}-\mathcal{M}_{Y}(f)\right) \varphi d x & =\int_{U}\left(f_{\epsilon}-g_{\epsilon}\right) \varphi d x+\int_{U}\left(g_{\epsilon}-\mathcal{M}_{Y}(g)\right) \varphi d x \\
& +\int_{U}\left(\mathcal{M}_{Y}(g)-\mathcal{M}_{Y}(f)\right) \varphi d x
\end{aligned}
$$

Se $I$ é um hipercubo em $\mathbb{R}^{n}$ tais que $U \subset I$, de (A.15) e (A.17) resulta que existe uma constante $C_{1}$ independente de $\epsilon$ e $\eta$ tais que

$$
\begin{aligned}
\left|\int_{U}\left(f_{\epsilon}-g_{\epsilon}\right) \varphi(x) d x\right| & \leq\left|\int_{I}\left(f_{\epsilon}-g_{\epsilon}\right) \varphi(x) d x\right| \leq\left(n(\epsilon)+n^{\prime}(\epsilon)\right) \epsilon^{n} \int_{I}|f(x)-g(x) \| \varphi(x)| d x \\
& \rightarrow \frac{|I|}{|Y|}\|f-g\|_{L^{1}(I)}\|\varphi\|_{L^{\infty}(U)} \leq C_{1} \eta\|\varphi\|_{L^{\infty}(U)} .
\end{aligned}
$$

De (A.17) tem-se

$$
\left|\int_{U}\left(\mathcal{M}_{Y}(g)-\mathcal{M}_{Y}(f)\right) \varphi d x\right| \leq C_{2} \eta\|\varphi\|_{L^{\infty}(U)},
$$

onde $C_{2}$ é uma constante independente de $\epsilon$ e $\eta$. Finalmente por (a)

$$
\int_{U}\left(g_{\epsilon}-\mathcal{M}_{Y}(g)\right) \varphi d x \rightarrow 0
$$

Consequentemente de (A.18) temos

$$
\int_{U}\left(f_{\epsilon}-\mathcal{M}_{Y}(f)\right) \varphi d x \rightarrow 0
$$

uma vez que $\eta$ é arbitrário, dessa forma concluimos a demonstração do Teorema. 


\section{APÊNDICE B}

\section{ALGUNS RESULTADOS SOBRE ESPAÇOS DE SOBOLEV}

Neste capítulo, pretendemos abordar algumas questões sobre os espaços de Sobolev $W^{m, p}(U)$, como são construidos e algumas propriedades desses espaços que são frequentemente usadas ao longo do texto. Para mais detalhes pode-se consultar por exemplo [32, 31, 13, 5, 28].

\section{B.1 Derivada fraca}

Seja $\varphi$ uma função real definida num aberto $U$ de $\mathbb{R}^{n}, \varphi$ é mensurável, e seja $\left(O_{i}\right)_{i \in I}$ a família de todos subconjuntos abertos de $O_{i}$ de $U$ tais que $\varphi=0$ quase sempre. Consideremos o subconjunto $O=\bigcup O_{i}$. Então $\varphi=0$ em $O$ e consequentemente

Definição B.1.1 Para uma função $\varphi: U \rightarrow \mathbb{R}$ o suporte da $\varphi$ denotado por $\operatorname{supp}(\varphi)$ é definido como sendo o subconjunto fechado de $U$,

$$
\operatorname{supp}(\varphi)=U \backslash O
$$

Se $\varphi$ é uma função contínua em $U$, então

$$
\operatorname{supp}(\varphi)=\overline{\{x \in O \mid \varphi(x) \neq 0\}}
$$

Se este conjunto for um conjunto compacto de $\mathbb{R}^{n}$ diremos que $\varphi$ possui suporte compacto.

Definição B.1.2 (Funções teste). Para $U \subset \mathbb{R}^{n}$, o conjunto

$$
C_{0}^{\infty}(U)=\left\{u \in C^{\infty}(U): \operatorname{supp}(u) \subset \subset U\right\}
$$


das funções suaves do suporte compacto. Tradicionalmente $\mathcal{D}(U)$ é frequentemente usado para denotar $C_{0}^{\infty}(U)$, isto é, $\mathcal{D}(U)$ é o espaço das funções teste.

Teorema B.1.3 Para $1 \leq p<\infty$ o espaço $\mathcal{D}(U)$ é denso em $L^{p}(U)$.

Demonstração. veja por exemplo [28, Proposição 6.]

Definição B.1.4 Definimos um multi-índice de $\alpha$ de ordem $k$ como uma n-upla $\alpha=\left(\alpha_{1}, \alpha_{2}, \ldots, \alpha_{n}\right)$ tal que:

$$
|\alpha|=\sum_{i=1}^{n} \alpha_{i} \quad \text { e } \quad \alpha !=\alpha_{1} ! \alpha_{2} ! \cdots \alpha_{n} !
$$

onde $\alpha_{i} \in \mathbb{N}^{n}$. O número $|\alpha|$ é a ordem do multi-índice $\alpha$. Se $|\alpha|>1$ e $x=\left(x_{1}, x_{2}, \ldots, x_{n}\right)$, então

$$
x^{\alpha}=x_{1}^{\alpha_{1}} x_{2}^{\alpha_{2}} \cdots x_{n}^{\alpha_{n}}
$$

Se $f \in C(U)$ denotamos

$$
D^{\alpha} f=\frac{\partial^{|\alpha|} f}{\partial x_{1}^{\alpha_{1}} \partial x_{2}^{\alpha_{2}} \cdots \partial x_{n}^{\alpha_{n}}}
$$

o operador de derivação quando a derivada mista do lado direito existe. $D^{\alpha} f=f$ quando $|\alpha|=0$. Temos que $D^{\alpha} f$ é uma função definida em $U$ que toma valores em $\mathbb{R}$. Assim, quando $f$ possui todas derivadas mistas de ordem $k$ escrevemos

$$
D^{k} f(x)=\left\{D^{\alpha} f(x): \alpha \text { é um multi-índice de ordem } k\right\}
$$

Definição B.1.5 (Derivada fraca). Suponha que $u \in L_{l o c}^{1}(U)$. Então $v^{\alpha} \in L_{l o c}^{1}(U)$ chama-se $\alpha$-ésima derivada fraca de u e escreve-se $v^{\alpha}=D^{\alpha} u$ se

$$
\int_{U} u(x) D^{\alpha} \phi(x) d x=(-1)^{|\alpha|} \int_{U} v^{\alpha} \phi(x) d x \quad \forall \phi \in C_{0}^{\infty}(U)
$$

Definição B.1.6 Seja $\left\{\varphi_{n}\right\}$ uma sequência $\mathcal{D}(O)$. Dizemos que a $\varphi_{n}$ converge para um elemento $\varphi \in \mathcal{D}(O)$, se e somente se

i) Existe um conjunto compacto $K \subset O$ tal que para todo $n \in \mathbb{N} \operatorname{supp}\left(\varphi_{n}\right) \subset K, n=1,2, \ldots$;

ii) Para todo $\alpha \in \mathbb{N}^{n}, D^{\alpha} \varphi_{n}$ converge uniformemente para $D^{\alpha} \varphi$ em $K$, quando $n \rightarrow \infty$.

\section{B.2 Distribuições}

Com base na definição anterior sabemos sobre a convergência em $C_{0}^{\infty}(U)$, assim podemos definir funcional contínuo em $C_{0}^{\infty}(U)$. Tal funcional é chamado distribuição, veremos também a sua convergência e algumas operações. 
Definição B.2.1 Seja $O \subset \mathbb{R}^{n}$. O funcional linear contínuo $T: \mathcal{D}(O) \rightarrow \mathbb{R}$ é dito distribuição em $O$, se e somente se

i) Té linear, isto é,

$$
\forall \lambda_{1}, \lambda_{2} \in \mathbb{R}, \varphi_{1}, \varphi_{2} \in \mathcal{D}(O) \quad T\left(\lambda_{1} \varphi_{1}+\lambda_{2} \varphi_{2}\right)=\lambda_{1} T\left(\varphi_{1}\right)+\lambda_{2} T\left(\varphi_{2}\right)
$$

ii) Té continua em sequência, isto é,

$$
\left(\varphi_{n} \rightarrow \varphi \quad \text { em } \quad \mathcal{D}(O)\right) \rightarrow\left(T\left(\varphi_{n}\right) \rightarrow T(\varphi)\right)
$$

Denotaremos por $\mathcal{D}^{\prime}(O)$ o conjunto das distribuições em $O$ e $T(\varphi)=\langle T, \varphi\rangle$.

Exemplo B.2.2 Seja $f \in L_{l o c}^{1}(O)$. O conjunto

$$
\left\langle T_{f}, \phi\right\rangle=\int_{O} f \phi d x, \quad \forall \phi \in \mathcal{D}(O)
$$

é uma distribuição.

De fato, $T_{f}$ é linear pois,

$$
\left\langle T_{f}, \phi_{1}+\lambda \phi_{2}\right\rangle=\int_{O} f(x)\left(\phi_{1}+\lambda \phi_{2}\right)(x) d x \int_{O} f(x) \phi_{1}(x) d x+\lambda \int_{O} f(x) \phi_{2}(x) d x=\left\langle T_{f}, \phi_{1}\right\rangle+\lambda\left\langle T_{f}, \phi_{2}\right\rangle,
$$

e $T_{f}$ é contínua, pois se $\phi_{j}$ é uma sequência convergindo para $\phi$ em $\mathcal{D}(O)$ tem-se

$$
\left|\left\langle T_{f}, \phi_{j}-\phi\right\rangle\right|=\left|\int_{\operatorname{supp}\left(\phi_{j}\right)} f\left(\phi_{j}-\phi\right) d x\right| \leq\|f\|_{L^{1}(K)} \sup _{K}\left|\phi_{j}-\phi\right| \rightarrow 0 .
$$

Onde $K$ é um conjunto compacto que contém o $\operatorname{supp}\left(\phi_{j}\right), \forall j$. Portanto a Definição B.2.1 logo $T_{f}$ é distribuição.

De acordo com [10], mostra-se que se $\left\langle T_{f}, \phi\right\rangle=\left\langle T_{g}, \phi\right\rangle$ para toda $\phi \in \mathcal{D}(O)$ e $f, g \in L_{L o c}^{1}(O)$. Então $f=g$ q.s. Com base na igualdade (B.1) podemos enunciar o seguinte teorema

Teorema B.2.3 Se $f \in L_{\text {Loc }}^{1}(O)$ é tal que

$$
\int_{O} f(x) \phi(x) d x=0, \quad \forall \phi \in \mathcal{D}(O) .
$$

Então $f=0$, q.s em $O$.

Demonstração. Veja por exemplo [28, Proposição 3].

Com base no Teorema B.2.3 segue que se $T$ é definido tal como em (B.1) mostra-se que $T_{f}=0$ se e somente se $f=0$, veja por exemplo [28, Proposição 4]. Esta observação nos induz a 
Definição B.2.4 Dizemos que a distribuição T é em $L_{\text {Loc }}^{1}(O)$ (respectivamente $L^{1}(O)$ ) se existe $f \in L_{\text {Loc }}^{1}(O)$ (respectivamente em $L^{1}(O)$ ) tal que $T=T_{f}$, onde $T_{f}$ é dado em (B.1).

Observação B.2.5 Suponha que $T \in \mathcal{D}^{\prime}(O)$ é como na Definição (B.2.4). Então a função $f$ está bem determinada em vertude do Teorema B.2.3. Por isso podemos identificar frequentemente $T$ com $f$, ou seja,

$$
\langle f, \phi\rangle=\left\langle T_{f}, \phi\right\rangle=\int_{O} f \phi d x
$$

Assim podemos identificar as funções localmente integráveis com o funcional $T_{f}$ dado em (B.1) e podemos considerar outros espaços de funções como $L^{p}(O), 1 \leq p \leq \infty, C^{k}(O), 1 \leq k \leq \infty$ como subespaços de $\mathcal{D}^{\prime}(O)$. Neste sentido as distribuições são denominadas funções generalizadas. A noção de convergência para sequência em $\mathcal{D}^{\prime}(O)$

Definição B.2.6 A sequência $\left\{T_{n}\right\}$ em $\mathcal{D}^{\prime}(O)$ diz-se converge (no sentido de destribuição) para um elemento $T \in \mathcal{D}^{\prime}(O)$, se e somente se

$$
\left\langle T_{n}, \varphi\right\rangle_{\mathcal{D}^{\prime}(O), \mathcal{D}(O)} \rightarrow\langle T, \varphi\rangle_{\mathcal{D}^{\prime}(O), \mathcal{D}(O)} \quad \varphi \in \mathcal{D}(O)
$$

A denotação para esta convergência é dada por

$$
T_{n} \rightarrow T \quad \text { em } \quad \mathcal{D}^{\prime}(O)
$$

Definição B.2.7 (Derivada da distribuição) Seja $T \in \mathcal{D}^{\prime}(O)$ para todo $i=1,2, \ldots$, n a derivada $\frac{\partial T}{\partial x_{i}}$ de $T$ em relação a $x_{i}$ é definido por

$$
\left\langle\frac{\partial T}{\partial x_{i}}, \varphi\right\rangle_{\mathcal{D}^{\prime}(O), \mathcal{D}(O)}=-\left\langle T, \frac{\partial \varphi}{\partial x_{i}}\right\rangle_{\mathcal{D}^{\prime}(O), \mathcal{D}(O)} \quad \forall \varphi \in \mathcal{D}(O) .
$$

É claro que $\frac{\partial T}{\partial x_{i}}, i=1,2, \ldots, n$ é linear pois o operador derivada é linear. Agora seja $\varphi_{n} \rightarrow 0$ em $\mathcal{D}(O)$ temos que $\frac{\partial \varphi_{n}}{\partial x_{i}} \rightarrow 0$ em $\mathcal{D}(O)$ o que mostra que $\frac{\partial T}{\partial x_{i}}$ é continua e portanto é uma distribuição. E além disso se $T_{n}$ converge para $T$ no sentido de distribuição então $\frac{\partial T_{n}}{\partial x_{i}}$ converge para $\frac{\partial T}{\partial x_{i}}$ no sentido de destribuição para $i=1,2, \ldots, n$, isto é,

$$
\left\langle T_{n}, \varphi\right\rangle \rightarrow\langle T, \varphi\rangle \quad \forall \varphi \in \mathcal{D}(O) \quad \text { e } \quad\left\langle\frac{\partial T}{\partial x_{i}}, \varphi\right\rangle \rightarrow\langle S, \varphi\rangle \quad \forall \varphi \in \mathcal{D}(O), \quad \text { então } \quad S=\frac{\partial T}{\partial x_{i}} .
$$

De fato,

$$
\left\langle\frac{\partial T}{\partial x_{i}}, \varphi\right\rangle=-\left\langle T, \frac{\partial \varphi}{\partial x_{i}}\right\rangle=-\lim \left\langle T_{n}, \frac{\partial \varphi}{\partial x_{i}}\right\rangle=\lim \left\langle\frac{\partial T_{n}}{\partial x_{i}}, \varphi\right\rangle=\langle S, \varphi\rangle \quad \forall \varphi \in \mathcal{D}(O)
$$


Observação B.2.8 Seja $f \in L^{1}(O)$. Suponhamos que $f$ é derivavel no sentido de destribuição $\frac{\partial f}{\partial x_{i}}$ é em $L^{1}(O)$. pelo considerações feita acima temos

$$
\int_{O} f \frac{\partial \varphi}{\partial x_{i}} d x=-\int_{O} \frac{\partial f}{\partial x_{i}} \varphi d x, \quad \forall \varphi \in \mathcal{D}(O)
$$

Esta observação juntamente com o Teorema B.2.3 diz em particular que se a função é da classe $C^{1}(O)$ a derivada no sentido de destribuição coincide com a derivada com a derivada usual.

\section{B.3 Espaços de $W^{m, p}(U)$}

O espaço Linear $W^{m, p}(U)$ é construido da seguinte forma. Uma função $u \in W^{m, p}(U)$ se $u \in L^{p}(U)$ e se todas as derivadas fracas de ordem menor ou igual a $m$ pertencem a $L^{p}(U)$, ou seja. Considere $U$ um domínio limitado em $\mathbb{R}^{n}$ e denotemos por $m$ a parte inteira de $s$ e por $\sigma$ a sua parte fracionária ou seja $s=m+\sigma$. Por [31] temos

Definição B.3.1 (Espaços de Sobolev) $W^{m, p}(U)$ é o espaço de todas as distribuições definidas em $\mathbb{R}^{n}$, tais que:

(a) $D^{\alpha} u \in L^{p}(U)$ para $|\alpha| \leq m$, quando $s=m$ é um inteiro não negativo.

(b) $\iint_{U \times U} \frac{\left|D^{\alpha} u(x)-D^{\alpha} u(y)\right|^{p}}{|x-y|^{n+\sigma p}} d x d y<\infty$, para $|\alpha|=m$, quando $s=m+\sigma$ é não inteiro e não negativo.

Definimos neste espaço a norma: Para o caso $(a)$

$$
\|u\|_{m, p, U}=\left(\sum_{|\alpha|<m} \int_{U}\left|D^{\alpha} u\right|^{p} d x\right)^{1 / p}
$$

e para o caso $(b)$

$$
\|u\|_{s, p, U}=\left(\|u\|_{m, p, U}^{p}+\sum_{|\alpha|=m} \iint_{U \times U} \frac{\left|D^{\alpha} u(x)-D^{\alpha} u(y)\right|^{p}}{|x-y|^{n+\sigma p}} d x d y\right)^{1 / p}
$$

O espaço $W^{m, p}(U)$ munido da norma (B.3) é um espaço de Banach e é separável [32, Teoremas 3.3 e 3.5]. No caso $p=2$ é um espaço de Hilbert munido do produto interno

$$
(u, v)_{W^{m, 2}(U)}=\int_{U} \sum_{|\alpha| \leq m} D^{\alpha} u(x) D^{\alpha} v(x) .
$$

Definição B.3.2 $H^{m, p}(U)$ é o completamento na norma (B.3) do espaço das funções $u: U \rightarrow \mathbb{R}$ da classe $C^{m}(U)$ tais que $D^{\alpha} u \in L^{p}(U), \operatorname{com}|\alpha| \leq m$. 
Prova-se que $W^{m, p}(U)=H^{m, p}(U)$ para $1 \leq p<\infty$ (veja por exemplo [32, Teorema 3.17]). No caso quando $p=2$ tem-se uma notação especial $H^{m, p}(U)=H^{m}(U)$. No caso particular em que $m=1 \mathrm{e}$ $p=12$ temos que $W^{1,2}(U)=H^{1}(U)$ é um espaço de Hilbert e defini-se como

$$
H^{1}(U)=\left\{u \mid u \in L^{2}(U), \frac{\partial u}{\partial x_{i}} \in L^{2}(U), \quad i=1,2, \ldots, n\right\},
$$

dotado do produto interno seguinte

$$
(u, v)_{H^{1}(U)}=(u, v)_{L^{2}(U)}+\sum_{i=1}^{n}\left(\frac{\partial u}{\partial x_{i}} \frac{\partial v}{\partial x_{i}}\right)_{L^{2}(U)}=\int_{U} u v+\sum_{i=1}^{n} \int_{U} \frac{\partial u}{\partial x_{i}} \frac{\partial v}{\partial x_{i}} \quad u, v \in H^{1}(U) .
$$

Uma norma associada é dado por

$$
\|u\|_{H^{1}(U)}=\left(\|u\|_{L^{2}(U)}^{2}+\sum_{i=1}^{n}\left\|\frac{\partial u}{\partial x_{i}}\right\|_{L^{2}(U)}\right)^{1 / 2}=\left(\|u\|_{L^{2}(U)}^{2}+\sum_{i=1}^{n}\|\nabla u\|_{L^{2}(U)}\right)^{1 / 2}
$$

onde $\nabla u$ é o gradiente de $u$ e é definido como $\nabla u=\left(\frac{\partial u}{\partial x_{1}}, \frac{\partial u}{\partial x_{2}}, \ldots, \frac{\partial u}{\partial x_{n}}\right)$.

\section{B.4 Imersões de Sobolev}

Muitas propriedades de espaços de Sobolev definidos em um domínio $U$ e em particular propriedades de imersão destes espaços dependem das propriedades de regularidade do domínio $U$, que geralmente são expressas por condições geométricas que podem ou não serem satisfeitas num dado domínio. Nessa ótica antes de enunciar os teoremas de imersão de Sobolev precisamos de algumas definições sobre essas propriedades geométricas de domínio $U$ que podem ser encontradas para uma leitura mais aprofundada em [31,32], pelo que

Definição B.4.1 Uma cobertura aberta $O$ de um conjunto $S \subset \mathbb{R}^{n}$ é dita localmente finita se todo conjunto compacto de $\mathbb{R}^{n}$ pode intersetar no máximo um número finito de elementos de $O$.

Definição B.4.2 Um dominio U satisfaz a Propriedade Lipschitz local se existe uma cobertura localmente finita $\Gamma$ por abertos $U$ tais que $\Gamma \cap U$ são gráficos de funções uniformemente Lipschitz.

Definição B.4.3 Seja U um domínio em $\mathbb{R}^{n}$. A fronteira dU é dita Lipschitz contínua existem constantes $a_{j}, 1 \leq j \leq n$ e para cada $x \in \partial U$ existe uma vizinhança $V$ de $x$ em $\mathbb{R}^{n}$ e novas coordenadas ortogonais $\left\{y_{1}, y_{2}, \ldots, y_{n}\right\}$ tais:

(a) Vé um hipercubo nestas novas coordenadas

$$
V=\left\{\left(y_{1}, y_{2}, \ldots, y_{n}\right):-a_{j}<y_{j}<a_{j}, 1 \leq j \leq n\right\}
$$


(b) Existe uma aplicação $\varphi: V^{\prime} \rightarrow \mathbb{R}$ Lipschitz contínua, onde $V^{\prime}$ é definida por

$$
V^{\prime}=\left\{\left(y_{1}, y_{2}, \ldots, y_{n-1}\right):-a_{j}<y_{j}<a_{j}, 1 \leq j \leq n-1\right\}
$$

tais que

$$
\begin{aligned}
& \left|\varphi\left(y^{\prime}\right)\right| \leq a_{n} / 2 \text { para todo } y^{\prime}=\left(y_{1}, y_{2}, \ldots y_{n-1}\right) \in V^{\prime} \\
& U \cap V=\left\{y=\left(y^{\prime}, y_{n}\right) \in V: y_{n}<\varphi\left(y^{\prime}\right)\right\} \\
& \partial U \cap V=\left\{y=\left(y^{\prime}, y_{n}\right) \in V: y_{n}=\varphi\left(y^{\prime}\right)\right\}
\end{aligned}
$$

Por outras palavras a Definição B.4.3 diz que em uma vizinhança de $x$ o domínio $U$ pertence ao gráfico da $\varphi$ e consequentemente a fronteira $\partial U$ é gráfico da $\varphi$.

Definição B.4.4 Uma função de $\varphi: V^{\prime} \rightarrow \mathbb{R}$ é dita Lipschitz contínua se existe uma constante positiva $K$ tal que

$$
|\varphi(x)-\varphi(y)| \leq K|x-y|, \quad \forall x, y \in V^{\prime},
$$

onde a constante K é chamada constante de Lipschitz.

Definição B.4.5 $C_{B}^{m}(U)$ é o espaço das funções $u \in C^{m}(U)$ tais que $D^{\alpha} u, 0 \leq|\alpha| \leq m$ é limitada em $U$. munido da norma

$$
\|u\|_{C_{B}^{m}(U)}=\max _{0 \leq|\alpha| \leq m} \sup _{x \in U}\left|D^{\alpha} u(x)\right| .
$$

$C_{B}^{m}(U)$ é um espaço de Banach e é maior que o espaço $C^{m}(\bar{U})$ em que os seus elementos não precisam ser necessariamente uniformemente contínuas, onde

$$
C^{m}(\bar{U})=C^{m}(U) \cap\left\{u, D^{\alpha} u \text { tem uma extensão contínua na } \bar{U}, 0 \leq|\alpha| \leq m\right\} .
$$

Antes de enunciar os teoremas de imersão de Sobolev vamos dar a seguinte noção. Sejam $Z_{1}$ e $Z_{2}$ espaços vetoriais normados. Dizemos que $Z_{1}$ é uma imersão em $Z_{2}$ se

$$
Z_{1} \subset Z_{2}
$$

O operador identidade $I$ é considerado como um operador que age $Z_{1}$ em $Z_{2}$, ou seja,

$$
\forall f \in Z_{1} \quad I(f)=f \quad I: Z_{1} \rightarrow Z_{2}
$$

que é possível graças a (B.9), chamaremos operador de imersão correspondendo a imersão (B.9).

Definição B.4.6 Sejam $Z_{1}$ e $Z_{2}$ espaços vetoriais dizemos que $Z_{1}$ é uma imersão contínua em $Z_{2}$ e escrevemos

$$
Z_{1} \hookrightarrow Z_{2}
$$


se em (B.9) o correspondente operador de imersão é contínua, isto é existe $C>0$ tal que $\forall f \in Z_{1}$

$$
\|f\|_{Z_{2}} \leq\|f\|_{Z_{1}}
$$

Teorema B.4.7 ( de Imersão de Sobolev) Seja U um dominio em $\mathbb{R}^{n}$ satisfazendo a propriedade de cone (veja [31, Definição 1.2.2.1]), j e m inteiros não negativos e $p$ satisfazendo $1 \leq p<\infty$. Então as imersões seguintes são contínuas

I) Se $m p<n$, então,

$$
W^{j+m, p}(U) \hookrightarrow W^{j, q}(U) \quad p \leq q \leq \frac{n p}{n-m p} \stackrel{\text { def }}{=} p^{*}
$$

ou

$$
\begin{gathered}
W^{m, p}(U) \hookrightarrow L^{q}(U) \quad p \leq q \leq p^{*} . \\
W^{j+m, p}(U) \hookrightarrow W^{j, q}(U), \quad p \leq q<\infty .
\end{gathered}
$$

III) Se $m p>n$, então,

$$
W^{j+m, p}(U) \hookrightarrow C_{B}^{j}(U)
$$

IV) Se $p(m-1)<n<m p$ e U tem a grande propriedade Lipschitz local, então,

$$
W^{j+m, p}(U) \hookrightarrow C^{j, \lambda}(\bar{U}), \quad 0<\lambda<1 .
$$

Onde $C^{j, \lambda}$ denota o espaço de Banach das restrições $\bar{U}$ das funções pertencentes a $C^{j, \lambda}\left(\mathbb{R}^{n}\right)$ equipado da seguinte norma induzida

$$
\|u\|_{C^{j, \lambda}}=\max _{0 \leq|\alpha| \leq m} \sup _{x \in U}\left|D^{\alpha} u(x)\right|+\max _{|\alpha| \leq k} \sup _{\substack{x, y \in \bar{U} \\ x \neq y}} \frac{\left|D^{\alpha} u(x)-D^{\alpha} u(y)\right|}{\|x-y\|^{p}} .
$$

Demonstração. Veja por exemplo [32, Teorema 5.2]

Teorema B.4.8 (Rellich- Kondrachov) Seja U um dominio limitado satisfazendo a propriedade de cone, $j$ e $m$ são inteiros não negativos, com $m \geq 1 e 1 \leq p<\infty$. Então para qualquer $j$ as imersões seguintes são compactas:

I) Se $m p<n$, então,

$$
W^{j+m, p}(U) \hookrightarrow W^{j, q}(U) \quad 1 \leq q<p^{*}
$$

II) Se $m p=n$, então,

$$
W^{j+m, p}(U) \hookrightarrow W^{j, q}(U) \quad 1 \leq q<\infty
$$


III) Se $m p>n$, então,

$$
W^{j+m, p}(U) \hookrightarrow C_{B}^{j}(U)
$$

IV) Se U satisfaz a grande propriedade Lipschitz local, então,

$$
\left\{\begin{array}{l}
W^{j+m, p}(U) \hookrightarrow C^{j}\left(U_{0}\right) \quad, \text { se } m p>n \\
W^{j+m, p}(U) \hookrightarrow C^{j, \lambda}\left(U_{0}\right), \text { se } m-1<\frac{n}{p}<m \quad \text { e } 0<\lambda<m-\frac{n}{p} .
\end{array}\right.
$$

Onde $U_{0}$ é um subdominio de $U$.

Demonstração. Veja por exemplo [32, Teorema 6.2]

\section{B.5 Espaço $W_{0}^{m, p}(U)$}

Por [31] a definição B.3.1 não se aplica para o conjunto $\mathcal{D}(U)$ pois o conjunto das funções $C_{0}^{\infty}(U)$ não é denso em $W^{m, p}(U)$ para $m>1$. Consequentemente o espaço dual de $W^{m, p}(U)$ não pode ser identificado como o espaço das distribuições em $U$. Motivados por esta razão introduz-se o seguinte espaço:

Definição B.5.1 Para $m>0$ denotemos por $W_{0}^{m, p}(U)$ o fecho de $\mathcal{D}(U)$ em relação a norma em $W^{m, p}(U)$. $O$ que equivale a dizer que $W_{0}^{m, p}(U)$ é o fecho em $W^{m, p}(U)$ de todas as distribuições com suporte compacto em $U$. Assim segue a extensão da definição B.3.1

Definição B.5.2 Para $m<0$ denotemos por $u \in W^{m, p}(U)$ o espaço dual de $W^{-m, q}(U)$ onde $p$ e $q$ são conjugados definidos pela relação (A.6).

Em especial para $p=2$ como fizemos antes $W_{0}^{m, p}(U)=H_{0}^{m}(U)$ também é espaço de Hilbert. Quando $s=1$ e temos

$$
W_{0}^{1,2}(U)=H_{0}^{1}(U)
$$

É claro que $H_{0}^{1}(U) \hookrightarrow H^{1}(U), \operatorname{logo} H_{0}^{1}(U)$ é um espaço de Hilbert com o produto interno (B.6).

As funções em $H_{0}^{1}(U)$ são caraterizados como sendo funções em $H^{1}(U)$ que se anulam na $\partial U$, mas precisamente $u \in H_{0}^{1}(U)$ são definidas quase sempre e a medida é nula na fronteira $\partial U$ e assim temos o seguinte resultado

Teorema B.5.3 Suponhamos que $u \in H^{1}(U)$. Então $u \in H_{0}^{1}(U)$ se e somente se $u=0$ quase sempre na fronteira dU Lipschitz contínua. Ou seja

$$
H_{0}^{1}(U)=\left\{u \mid u \in H^{1}(U), \text { e } u=0 \text { quase sempre na } \partial U\right\}
$$

Demonstração. Veja por exemplo [13, Teorema 8.12]. 
Teorema B.5.4 ( de Green) Suponhamos que a fronteira $\partial U$ é Lipschitz contínua. Então para todo $u, v \in$ $H^{1}(U)$ temos

$$
\int_{U} u \frac{\partial v}{\partial x_{i}} d x=-\int_{U} \frac{\partial v}{\partial x_{i}} v d x+\int_{\partial U} \gamma(u) \gamma(v) v_{i} d \sigma
$$

onde $v=\left(v_{1}, v_{2}, \ldots v_{n}\right)$ é o vetor normal exterior a $U$ e $\gamma(v)$ é o operador traço veja [5, Teorema 3.28] de v na fronteira $\partial U$

Proposição B.5.5 (Desigualdade de Poincaré) Existe uma constante $C_{U}$ tal que

$$
\|u\|_{L^{2}(U)} \leq C_{U}\|\nabla u\|_{L^{2}(U)}, \quad \forall u \in H_{0}^{1}(U)
$$

A constante $C_{U}$ é uma constante que depende apenas de $U$.

Demonstração. Veja por exemplo [5, Proposição 3.35].

Os teoremas de imersão de Sobolev continuam válidas para $W^{1, p}(U)$ sem nenhuma pré-condição para a fronteira $\partial U$ e temos

Teorema B.5.6 As seguintes inclusões são verdadeiras:

i) Se $1 \leq p<\infty \quad W_{0}^{1, p}(U) \hookrightarrow L^{q}(U)$, com $\left\{\begin{array}{l}\left.\text { imersão compacta para } q \in] 1, p^{*}\right], \quad p^{*}=\frac{n p}{n-p} \\ \text { imersão contínua para } q=p^{*} ;\end{array}\right.$

ii) Se $p=n \quad W_{0}^{1, p}(U) \hookrightarrow L^{q}(U)$, com imersão compacta para $q \in[1,+\infty]$;

iii) Se $p>n \quad W_{0}^{1, p}(U) \hookrightarrow C^{0}(\bar{U})$, com imersão compacta .

Em geral para $m>1$ e $1 \leq p<\infty$ para o espaço $W^{m, p}(U)$ e segundo a definição B.5.1 mostra-se que

$$
W_{0}^{m, p}(U)=\left\{u \in W^{m, p}(U): u=D(u)=\cdots=D^{m-1}(u)=0 \text { na } \partial U\right\} .
$$

O espaço $H_{0}^{1}(U)$ é muito importante no estudo de problemas elípticos e conforme a definição B.5.2. Temos

Definição B.5.7 o espaço $H^{-1}(U)$ é um espaço de Banach definido por

$$
H^{-1}(U)=\left(H_{0}^{1}(U)\right)^{*}
$$

equipado com a norma

$$
\|F\|_{H^{-1}(U)}=\sup _{H_{0}^{1}(U) \backslash\{0\}} \frac{\left|\langle F, u\rangle_{H^{-1}(U), H_{0}^{1}(U)}\right|}{\|u\|_{H_{0}^{1}(U)}} .
$$


Observação B.5.8 Suponhamos que a fronteira dU é Lipschitz contínua, tem-se que

$$
L^{2}(U) \hookrightarrow H^{-1}(U)
$$

com imersão compacta.

Para finalizar esta seção observemos que para $1 \leq p<\infty$ o espaço $L^{p}(U)$ pode ser identificado com o seu dual $L^{q}(U), p$ e $q$ são conjugados e que pelo Teorema B.1.3 o espaço $\mathcal{D}(U)$ é denso em $L^{p}(U)$, e juntamente com o fato de que $H_{0}^{1}(U) \hookrightarrow H^{1}(U)$, os Teoremas de Imersão B.4.7 e B.4.8 e a Observação B.5.8 temos que as seguintes imersões são compactas

$$
H_{0}^{1}(U) \hookrightarrow H^{1}(U) \hookrightarrow L^{2}(U) \hookrightarrow H^{-1}(U)
$$




\section{APÊNDICE C}

\section{TEOREMA DE LAX-MILGRAM}

Neste capítulo, pretendemos enunciar o Teorema de Lax-Milgram que garante a existência e unicidade de alguns problemas de Equações Diferenciais Parciais, ele estabelece que cada funcional linear definido num espaço de Hilbert $\mathcal{H}$ está associado a um único elemento em $\mathcal{H}$ de maneira que este funcional pode ser representado por uma forma bilinear e contínua veja [5, 13].

\section{C.1 Formas bilineares em espaços de Banach}

Definição C.1.1 Seja $V$ um espaço de Banach. Uma aplicação $a: V \times V \rightarrow \mathbb{R}$ é uma forma bilinear em $V$ se e somente se para qualquer $u \in V$ fixado as seguintes aplicações

$$
\begin{aligned}
& a(u, \cdot): u \in V \mapsto a(u, v) \in \mathbb{R} \\
& a(\cdot, u): u \in V \mapsto a(u, v) \in \mathbb{R}
\end{aligned}
$$

sao lineares.

Definição C.1.2 A aplicação $a: V \times V \rightarrow \mathbb{R}$ é limitada em $V$ se e somente se existe uma constante $C>0$ tal que

$$
|a(u, v)| \leq C\|u\|_{V}\|v\|_{V}
$$

Proposição C.1.3 Seja $a: V \times V \rightarrow \mathbb{R}$ uma forma bilinear. Então a é limitada se e somente se a é contínua em $V \times V$.

Prova. $(\Rightarrow)$ Suponhamos que vale (C.1). Então para $(u, v)$ e $\left(u_{0}, v_{0}\right) \in V \times V$ temos

$$
\begin{aligned}
\left|a(u, v)-a\left(u_{0}, v_{0}\right)\right| & =\left|a(u, v)-a\left(u, v_{0}\right)+a\left(u, v_{0}\right)-a\left(u_{0}, v_{0}\right)\right|=\left|a\left(u, v-v_{0}\right)+a\left(u-u_{0}, v\right)\right| \\
& \leq\left|a\left(u, v-v_{0}\right)\right|+\left|a\left(u-u_{0}, v\right)\right| \leq C\|u\|_{V}\left\|v-v_{0}\right\|_{V}+C\left\|u-u_{0}\right\|_{V}\|v\|_{V},
\end{aligned}
$$


que nos dá a continuidade de $a$ em $V \times V$.

$(\Leftarrow)$ Suponhamos que $a$ é contínua então para qualquer $\varepsilon>0$ existe $\delta>0$ talque

$$
\|w\|_{V}<\delta,\|z\|_{V}<\delta \quad \Longrightarrow|a(w, z)|<\varepsilon
$$

Agora pela linearidade, temos para todo $u, v \in V \backslash\{0\}$ temos

$$
\frac{|a(u, v)|}{\|u\|_{V}\|v\|_{V}}=\left|a\left(\frac{u}{\|u\|_{V}}, \frac{v}{\|v\|_{V}}\right)\right|
$$

introduzindo $w$ e $z$ como se segue

$$
w=\frac{\delta}{2} \frac{u}{\|u\|_{V}}, \quad z=\frac{\delta}{2} \frac{v}{\|v\|_{V}}
$$

É claro que

$$
\|w\|_{V}=\frac{\delta}{2}<\delta, \quad\|z\|_{V}=\frac{\delta}{2}<\delta
$$

Escrevendo (C.2) para $w$ e $z$ definidos em (C.4). Temos

$$
\left|a\left(\frac{u}{\|u\|_{V}}, \frac{v}{\|v\|_{V}}\right)\right|=\frac{4}{\delta^{2}}|a(w, z)| \leq \frac{4}{\delta^{2}} \varepsilon
$$

que junto com (C.3) dá-nos que

$$
\frac{|a(u, v)|}{\|u\|_{V}\|v\|_{V}}=\left|a\left(\frac{u}{\|u\|_{V}}, \frac{v}{\|v\|_{V}}\right)\right| \leq \frac{4}{\delta^{2}} \varepsilon
$$

e tomando $C=\frac{4}{\delta^{2}}$ segue (C.1) como queriamos.

Definição C.1.4 A forma bilinear a em $V$ é simétrica se e somente se

$$
a(u, v)=a(v, u), \quad \forall u, v \in V
$$

E positiva se e somente se

$$
a(u, u) \geq 0, \quad \forall u \in V .
$$

Definição C.1.5 A forma bilinear a é dita $V$ - elíptica ou coerciva em $V$ com a constante $\alpha_{0}$ se existe a constante $\alpha_{0}>0$ tal que

$$
a(u, u) \geq \alpha_{0}\|u\|_{V^{\prime}}^{2} \quad \forall u \in V .
$$




\section{C.2 Teorema de Lax-Milgram}

Seja a uma forma bilinear em um espaço de Hilbert $\mathcal{H}$ e $F \in \mathcal{H}^{*}$ onde $\mathcal{H}^{*}$ é o espaço dual de $\mathcal{H}$. Consideremos o seguinte problema

$$
\begin{cases}\text { Encontrar } u \in \mathcal{H} & \text { tal que } \\ a(u, v)=\langle F, v\rangle_{\mathcal{H}^{*}, \mathcal{H}} & \forall v \in \mathcal{H}\end{cases}
$$

A equação (C.5) chamaremos equação variacional e $v \in \mathcal{H}$ chamaremos função teste. O teorema de Lax-Milgram dá-nos condições sobre a existência e unicidade das soluções da equação (C.5) e basea-se fundamentalmente no teorema de Representação de Riez para o dual de um espaço de Hilbert que enunciamos a seguir

Teorema C.2.1 (de representação de Riez) Seja $\mathcal{H}$ um espaço de Hilbert e $F \in \mathcal{H}^{*}$. Então existe uma única $\tau F \in \mathcal{H}$ tal que

$$
\langle F, v\rangle_{\mathcal{H}^{*}, \mathcal{H}}=(\tau F, v)_{\mathcal{H}} \quad \forall v \in \mathcal{H}
$$

Além disso, a aplicação injetora

$$
\tau: F \in \mathcal{H}^{*} \mapsto \tau F \in \mathcal{H}
$$

é uma isometria (isometria de Riez), isto é, satisfaz

$$
\|\tau F\|_{\mathcal{H}}=\|F\|_{\mathcal{H}^{*}}
$$

Pelo exposto acima já estamos em condições de enunciar o seguinte resultado geral

Teorema C.2.2 (Lax-Milgram) Seja a uma forma bilinear e contínua em um espaço de Hilbert $\mathcal{H}$ e $F \in \mathcal{H}^{*}$. Assumimos que a é coercivo com constante $\alpha_{0}$. Então a equação variacional (C.5) tem solução única $u \in \mathcal{H}$. Além disso,

$$
\|u\|_{\mathcal{H}} \leq \frac{1}{\alpha_{0}}\|F\|_{\mathcal{H}^{*}}
$$

Demonstração. veja por exemplo [5, Teorema 4.6].

Se a forma bilinear $a$ é simétrica então $a(u, v)$ é um produto interno equivalente a $(\cdot, \cdot)_{\mathcal{H}}$ e portanto é uma consequência do Teorema C.2.1. Além disso, a equação variacional (C.5) pode ser caracterizado como um ponto mínimo de um funcional adequado. Veja [5, pág. 68] 


\section{APÊNDICE D}

\section{GRAU TOPOLÓGICO DE LERAY \& SCHAUDER}

Neste capítulo pretendemos abordar algumas questões sobre grau topológico de Leray \& Schauder suas propriedades, Teorema de Schauder sobre ponto fixo que garante a existência soluções de uma equação diferencial parcial elíptico. Para uma leitura mais aprofundada sugerimos [22, 14, 7, 12].

\section{D.1 Aplicações Leray \& Schauder}

Os conjuntos $X$ e $Y$ sempre denotaram espaços de Banach munidos da norma $\|\cdot\|$, e $U$ um subconjunto do espaço de Banach $X$.

Definição D.1.1 Seja $U \subset X$. Diremos que U é compacto se qualquer sequência de pontos de U contém uma subsequência que converge para algum ponto de $U$.

Definição D.1.2 Seja $X, Y$ espaços de Banach, $U \subset X$. Diremos que uma aplicação $f: U \rightarrow Y$ é compacta se

i) fé contínua;

ii) $\overline{f(A)}$ é um conjunto compacto para todo conjunto aberto e limitado $A \subset U$.

Equivalentemente fé contínua e $f(U)$ é relativamente compacto, isto é todo suconjunto fechado e limitado de U temos que $\overline{f(A)}$.

Denotaremos por $\mathcal{K}(U, Y):=$ o conjunto das aplicações compactas de $U$ em $Y$ e escreveremos simplesmente $\mathcal{K}(U)$. 
Definição D.1.3 Uma aplicação $f: U \rightarrow X$ diz-se de Leray \& Schauder que abreviadamente escreveremos L-S se:

i) $\Phi(x) \neq 0, \forall x \in \partial U$

ii) $\Phi$ é uma perturbação completamente contínua da identidade, isto é $\Phi=I-f$ e é completamente contínua.

Vamos provar algumas propriedades das aplicações L-S:

Proposição D.1.4 Seja U um subconjunto limitado de X e $f: U \rightarrow X$ uma aplicação L-S. Então

i) $f(A)$ é fechado para todo conjunto fechado $A \subset \bar{U}$;

ii) $f^{-1}(K)$ é compacto para todo conjunto compacto de X.

Demonstração. Seja $\Phi=I-f$

i) Assuma que $\left\{x_{n}: n \in \mathbb{N}\right\} \subset X$ e $\left\{f\left(x_{n}\right)\right\}$ converge para um ponto $p \in X$. Como $\Phi$ é completamente contínuo e $\bar{V}$ é um conjunto aberto e limitado, existe uma subsequência $\left\{x_{n j}\right\}_{j}$ tais que $f\left(x_{n j}\right)$ converge para algum $y \in X$. Temos que

$$
y+p=\lim _{j \rightarrow+\infty} x_{n j}
$$

Mas por hipótese temos que $A$ é um conjunto fechado então $p+y \in A$ e

$$
f(y+p)=f\left(\lim x_{n j}\right)=\lim f\left(x_{n j}\right)=p, \quad \text { assim } \quad p \in f(A) .
$$

ii) Seja $\left\{x_{n}: n \in \mathbb{N}\right\} \subset f^{-1}(K)$. Existe uma subsequência $\left\{x_{n j}\right\} \subset f^{-1}(K)$ tais que $\left\{\Phi\left(x_{n j}\right)\right\}_{j}$ converge para algum $y \in X$. Temos que $x_{n j}-\Phi\left(x_{n j}\right)=f\left(x_{n j}\right) \in K$ e como $K$ é um conjunto compacto a subsequência $x_{n j}-\Phi\left(x_{n j}\right)$ converge para algum $p \in X$. Assim

$$
\lim _{j \rightarrow+\infty} x_{n j}=\lim _{j \rightarrow+\infty}\left(x_{n j}-\Phi\left(x_{n j}\right)+\Phi\left(x_{n j}\right)\right)=y+p .
$$

Como $f^{-1}(K)$ é um conjunto fechado, temos que $p+y \in f^{-1}(K)$ e portanto $f^{-1}(K)$ é um conjunto compacto.

Proposição D.1.5 Seja U é um conjunto fechado e limitado em X e $f: \bar{U} \rightarrow X$ uma aplicação L-S injetora, então a aplicação $f^{-1}: f(\bar{U}) \rightarrow$ X é uma aplicação L-S.

Demonstração. Seja $\Phi=I-f$. Temos que

$$
I=\Phi+f=\left(\Phi \circ f^{-1}+I\right) \circ f .
$$


Assim vemos que $f^{-1}=\Phi \circ f^{-1}+I$.

Primeiro vamos mostrar que $\Phi \circ f^{-1}$ é relativamente compacto para todo $A \subset f(\bar{U})$ limitado. Com efeito $f^{-1}(A) \subset \bar{U}$ é limitado e $\Phi$ é compacto então $\Phi \circ f^{-1}(A)$ é relativamente compacto.

Para provar que $\Phi \circ f^{-1}$ é contínua consideremos a sequência $\left\{y_{n}: n \mathbb{N}\right\} \subset f(\bar{U})$ convergindo para $y \in f(\bar{U})$. Existe um conjunto $\left\{x_{n}: n \in \mathbb{N}\right\} \subset \bar{U}$ e um ponto $x \in \bar{U}$ tais que

$$
y_{n}=f\left(x_{n}\right) \quad \text { e } \quad y=f(x)
$$

É suficiente mostrar que

$$
x_{n}=f^{-1}\left(y_{n}\right) \rightarrow x=f^{-1}(y) .
$$

Pela Proposição D.1.4 e como $\left\{y_{n}: n \in \mathbb{N}\right\}$ é relativamente compacto vemos que o conjunto $\left\{f^{-1}\left(y_{n}\right)\right.$ : $n \in \mathbb{N}\}$ é também relativamente compacto. E consequentemente podemos extrair subsequência tal que $\left\{x_{n j}\right\}$ converge para algum ponto $a \in \bar{U}$. Agora usando a continuidade de $f$ em $a$ temos que

$$
f(a)=\lim _{j \rightarrow+\infty} f\left(x_{n j}\right)=y=f(x)
$$

e como $f$ é injetor, concluimos que $a=x$.

\section{D.2 Grau topológico de Leray \& Schauder}

Definição D.2.1 Seja $U \subset X$ e $\mathscr{H}: \bar{U} \times[0,1] \rightarrow X$. Diremos que $\mathscr{H}$ é uma homotopia da transformação compacta em U se

i) $\mathscr{H}(\cdot, t)$ é $\mathcal{K}(U)$ para todo $t \in[0,1]$;

ii) Para todo $\varepsilon>0$ e todo conjunto limitado $L \subset M$ existe $\delta>0$ tal que $\|\mathscr{H}(x, t)-\mathscr{H}(x, s)\|<\varepsilon$ sempre que $x \in L e|s-t|<\delta$.

Definição D.2.2 A tripla $(\Phi, U, y)$ é admíssivel se $f$ L-S e $y \in X$ com $y \notin f(\partial U)$ onde $\partial U$ é a fronteira do subconjunto limitado $U \subset X$.

A esta tripla admissível $(\Phi, U, y)$, existe uma função $D$ definida em $\mathbb{Z}$ veja [22, pág. 56] pelo que

Teorema D.2.3 Existe uma única função $D$ definida no conjunto de todas triplas admissíveis $(\Phi, U, y)$ determinada pelas seguintes propriedades:

(D1) $D(I, U, y)=1$ para $y \in U$;

(D2) $D(\Phi, U, y) \neq 0$ o que implica que $y \in \Phi(\partial U)$ então existe $x \in U$ tal que $\Phi(x)=y$; 
(D3) $D(\Phi, U, y)\left(\Phi, U_{1}, y\right)+D\left(\Phi, U_{2}, y\right)$ sempre que $U_{1}$ e $U_{2}$ forem subconjuntos disjuntos de $U$ tais que $y \notin \Phi\left(\bar{U} \backslash\left(U_{1} \cup U_{2}\right)\right) ;$

(D4) $D\left(\Phi_{t}, U, y\right)$ é independente de tse $\Phi_{t}$ é uma homotopia contínua tal que y $\notin \Phi_{t}(\partial U)$ para todo $t \in[0,1]$.

Teorema D.2.4 (Invariância sob homotopia) Suponhamos que $\mathscr{H}: \bar{U} \times[0,1] \rightarrow$ Xé uma homotopia de uma transformação compacta em $\bar{U}$. O conjunto

$$
\Phi_{t} \stackrel{\text { def }}{=} I-\mathscr{H}(\cdot, t)
$$

para $t \in[0,1]$ e suponhamos que $y \notin \Phi_{t}(\partial U)$ para todo $t \in[0,1]$. Então $D\left(\Phi_{t}, U, y\right)$ é independente de $t$.

Definição D.2.5 O número $D(\Phi, U, y)$ definido no Teorema D.2.3 chama-se grau topológico de Leray \& Schauder da aplicação $\Phi$.

Observação D.2.6 Se $X=\mathbb{R}^{n}$ então $D(\Phi, U, y)$ é o grau topológico de Brouwer em $\mathbb{R}^{n}$ e $\Phi \in C\left(\bar{U}, \mathbb{R}^{n}\right)$ com $y \notin \Phi(\partial U)$ e escreve-se $d(\Phi, U, y)$.

O grau topológico de Brouwer pode ser extendido para aplicações diferenciáveis [7, 14, 22].

Definição D.2.7 Seja $f: U \rightarrow \mathbb{R}^{n}$ de classe $C^{k}(U), k \geq 1$. O ponto $x_{0} \in U$ é um ponto crítico de $f$ se $J_{f}\left(x_{0}\right)=0$, onde $J_{f}\left(x_{0}\right)=\operatorname{det}\left(f^{\prime}\left(x_{0}\right)\right)=\operatorname{det}\left(\frac{\partial f_{i}}{\partial x_{j}}\right)$. E $f\left(x_{0}\right)$ é o valor crítico da $f$. Denotaremos por $S_{f}(U)$ ou simplesmente $S_{f}$ ao conjunto dos pontos de $U$ para os quais $J_{f}\left(x_{0}\right)=0$, ou seja

$$
S_{f}(U)=\left\{x \in U: J_{f}\left(x_{0}\right)=0\right\}
$$

Definição D.2.8 O ponto $y \in \mathbb{R}^{n}$ é valor regular de $f: \bar{U} \rightarrow \mathbb{R}^{n}$ se $f^{-1}(y) \cap S_{f}(U)=\emptyset$. Caso contrário chamaremos valor singular.

Lembremos que se $\bar{U}$ é compacto então $f^{-1}(\{y\})$ é fechado e que $x \in f^{-1}(\{y\})$ temos que $J_{f}(x) \neq 0$, então pelo Teorema da Aplicação Inversa [37]. Logo existe uma vizinhança $\mathbf{V}$ de $x$ e uma vizinhança $\mathbf{W}$ de $y$, isto é $\left.f\right|_{\mathbf{V}}: \mathbf{V} \rightarrow f(\mathbf{V})=\mathbf{W}$ é um defeomorfismo o que implica que $f^{-1}(\{y\})$ é finito.

Definição D.2.9 Seja $U \subset \mathbb{R}^{n}$ aberto e limitado, $f \in C^{1}\left(\bar{U}, \mathbb{R}^{n}\right)$ e $y \in \mathbb{R}^{n} \backslash f\left(\partial U \cup S_{f}\right)$. Definimos o Grau topológico de Brouwer de $f$ em relação a $U$ e y como sendo o número inteiro

$$
d(f, U, y)=\sum_{x \in f^{-1}(y)} \operatorname{sign}\left(J_{f}\left(x_{0}\right)\right),
$$

onde sign é a função sinal que é definida por:

$$
\operatorname{sign}(y)=\left\{\begin{array}{c}
+1, \quad \text { se } y>0 \\
-1, \text { se } y<0
\end{array}\right.
$$


É claro que da definição D.2.9 segue que se $f$ é a aplicação identidade, então

$$
d(I, U, y)=\left\{\begin{array}{lll}
1, & \text { se } & y \in U \\
0, & \text { se } & y \notin U .
\end{array}\right.
$$

Afim de enunciar as propriedades de grau topológico de Leray \& Schauder cujas demonstrações podem ser encontradas por exemplo em $[14,22,7]$ definamos

$$
\mathcal{K}_{1}(U) \stackrel{\text { def }}{=}\{\Phi: \Phi=I-f, f \in \mathcal{K}(U)\} .
$$

Teorema D.2.10 As seguintes afirmações são verdadeiras:

(5) $D(I, U, y)=0$ o que implica que $y \notin \Phi(\partial U)$;

(6) $D(\Phi, U, y)=D(\Phi, \widetilde{U}, y)$ para todo subconjunto $\widetilde{U} \subset U$ existe $x \in U \backslash \widetilde{U}$ tais que $\Phi(x) \neq y$.

(7) Suponhamos que $\Phi, \Psi \in \mathcal{K}_{1}(U),\left.\Phi\right|_{\partial U}=\left.\Psi\right|_{\partial U}$ e $y \notin \Phi(\partial U)$. Então $D(\Phi, U, y)=D(\Psi, U, y)$;

(8) Suponhamos que $\Phi \in \mathcal{K}_{1}(U), y \notin \Phi(\partial U)$ e seja $p \in X$. Então $D(\Phi, U, y)=D(\Phi-p, U, y-p)$

O grau de Leray \& Schauder provou ser uma ferramenta muito poderosa para o estudo dos pontos fixos das aplicações compactas e completamente contínuas. E tem tido aplicações na investigação da existencia de soluções de equações diferenciais [22, 14].

No que se segue assumimos que $X$ é um espaço de Banach uniformemente convexo, isto é, $X$ é um espaço de Banach munido da norma estritamente convexa,

$$
\|\lambda x+(1-\lambda) y\| \leq \lambda\|x\|+(1-\lambda)\|y\|
$$

para todo $x, y \in X$ linearmente independentes e $\lambda \in(0,1)$. Espaços de Hilbert são uniformemente convexos. Veja por exemplo [32, Corolário 2.29]

Teorema D.2.11 (Aplicações ímpar) Seja $U \subset X$ conjunto aberto, limitado, simétrico e contendo zero. Suponhamos que a aplicação $\Phi \in \mathcal{K}_{1}(U)$ é tal que $\Phi(-u)=-\Phi(u)$, com $u \in \partial U$ e $0 \notin \Phi(\partial U)$. Então $D(\Phi, \mathcal{U}, 0)$ é um número impar.

Demonstração. Veja por exemplo [14, Teorema 7.19 ].

Proposição D.2.12 Seja X um espaço de Banach $T \in \mathcal{K}(X)$, completamente contínuo e $L=I-T$. Então

a) Seja $M=I-M_{0}$, com $M_{0} \in \mathcal{K}(X)$ completamente contínua. supomos que L e $M$ são injetoras

$$
D(L M, U, 0)=D(T, U, 0) D(M, U, 0),
$$

para todo subconjunto aberto $U \subset X$ tais que $0 \in \Omega$. 
b) Seja $X=\bigoplus_{i=1}^{m} X_{i}$ uma soma direta de subespaços topológicos $X_{1}, X_{2}, \ldots, X_{m}$. E suponhamos que Lé injetora. Então

$$
D\left(T, B_{1}(0) \cap X_{i}, 0\right)=\prod_{i=1}^{m} D\left(\left.T\right|_{X_{i}}, B_{1}(0) \cap X_{i}, 0\right)
$$

Demonstração. veja por exemplo [22, Proposição 8.4]

Teorema D.2.13 Seja X um espaço de Banach real e $T \in \mathcal{K}(\bar{U})$, completamente contínuo, $\lambda \neq 0$ e $\lambda^{-1}$ não é auto-valor de L. Seja $U \subset X$ um subconjunto aberto e limitado e $0 \in \Omega$. Então

$$
D(I-\lambda T, U, 0)=(-1)^{\beta(\lambda)}
$$

onde $\beta(\lambda)$ é a soma das multiplicidades algébricas dos auto valores $\mu$ satisfazendo $\mu \lambda>1$. Se não existe auto-valores temos que $\beta(\lambda)=0$

Lembremos que se $\mu$ é um auto-valor de $L$, então a multiplicidade algébrica $m(\lambda)$ de $\mu$ é definido pela fórmula

$$
m(\mu)=\operatorname{dim} \bigcup_{n \geq 1} \operatorname{ker}\left[(\mu I-T)^{n}\right]
$$

Demonstração. veja por exemplo [22, Teorema 8.10]

No Teorema D.2.13 $T: \bar{U} \rightarrow X$ significa que o operador $T$ completamente contínuo não possui possui pontos fixos na $\partial U$. E para cada operador $T$ podemos associar um inteiro ind $(T, U)$ que chamaremos de índice de ponto fixo de T em U que nos dá uma caracterização sobre a existência e a multiplicidade algébrica de pontos fixos [12].

Definição D.2.14 (Ponto fixo isolado) Seja $T: U \rightarrow X$. Um ponto fixo $u^{*}$ é isolado se existir uma vizinhança $U_{0}$ de $u^{*}$ tal que o único ponto fixo de $T$ em $U_{0}$ é $u^{*}$.

Observação D.2.15 Com base na propriedade (6) do Teorema D.2.10 e nas condições do Teorema D.2.13 temos que a definição D.2.14 independe da escolha da vizinhança $U_{0}$ de $u^{*}$ [12].

De fato,

Suponhamos que $\widetilde{U}_{0}$ seja uma outra vizinhança de $u^{*}$ tal que $T(u) \neq u$ para todo $u \in \widetilde{U}_{0} \backslash\left\{u^{*}\right\}$ então $T$ não possui pontos fixos em ambos conjuntos $U_{0} \backslash\left(\widetilde{U}_{0} \cap U_{0}\right)$ e $\widetilde{U}_{0} \backslash\left(\widetilde{U}_{0} \cap U_{0}\right)$. Consequentemente

$$
\operatorname{ind}\left(T, U_{0}\right)=\operatorname{ind}\left(T, \widetilde{U}_{0} \cap U_{0}\right)=\operatorname{ind}\left(T, \widetilde{U}_{0}\right)
$$

E portanto temos 
Definição D.2.16 (Índice de ponto fixo isolado) O indice de T no ponto fixo isolado $u^{*}$ em uma vizinhança suficientemente pequena $U_{0}$ é definido por

$$
\operatorname{ind}\left(T, u^{*}\right) \stackrel{\text { def }}{=} \operatorname{ind}\left(T, U_{0}\right) \text {. }
$$

Definição D.2.17 (Derivada de Fréchet) Seja $U \subset X$ a aplicação $F: U \rightarrow Y$, onde $X$ e $Y$ são espaços normados é dita diferenciável a Fréchet no ponto $x_{0}$ se $x_{0}$ é um ponto interior de A e existe uma aplicação linear $L: X \rightarrow Y$ satisfazendo

$$
\lim _{x \rightarrow x_{0}} \frac{\left\|F(x)-F\left(x_{0}\right)-L\left(x-x_{0}\right)\right\|}{\left\|x-x_{0}\right\|_{X}}=0 .
$$

A derivada de Fréchet de $F$ em $x_{0}$ e denotar-se-à por $d F\left(x_{0}\right)$ ou simplesmente $F^{\prime}\left(x_{0}\right)$. Equivalentemente,

Definição D.2.18 Seja $U \subset X$ a aplicação $F: U \rightarrow Y$, onde $X$ e $Y$ são espaços normados. Dizemos que aplicação $T: X \rightarrow$ Yé a derivada de Fréchet de F em $x$ se para todo $\varepsilon>0$ existe $\delta>0$ tal que

$$
\|F(x+h)-F(x)-L(h)\|_{Y} \leq \varepsilon\|h\|_{X}
$$

para todo h sempre que $\|h\|_{X} \leq \delta$.

Finalmente, as condições ind $\left(T, u^{*}\right)$ ou ind $(T, U)$ são essencias para aplicações e algumas das suas aplicações são dadas pelos seguintes resultados [12]

Teorema D.2.19 Se U é um domínio convexo e $L=I-T$ e é o grau de Leray \& Schauder tal que

$$
T(\bar{U}) \subset \bar{U},
$$

então ind $(T, U)=1$.

Teorema D.2.20 (Sobre o ponto fixo de Schauder) Seja $U \subset X$ um conjunto fechado limitado e contendo a origem em seu interior e seja $T \in \mathcal{K}(U)$ tal que $T(U) \subset U$. Então $f$ tem um ponto fixo.

Teorema D.2.21 Se $T \in \mathcal{K}(U)$ é uma aplicação Fréchet diferenciável em um ponto fixo $u^{*}$ de $T$ e se o núcleo de $I-T^{\prime}\left(u^{*}\right)$ é tal que $\mathscr{N}\left(I-T^{\prime}\left(u^{*}\right)\right)=\{0\}$, então o ponto fixo $u^{*}$ é isolado e $\operatorname{ind}\left(T, u^{*}\right)=1$. 


\section{REFERÊNCIAS BIBLIOGRÁFICAS}

[1] A. Jiménez-Casas and A. Rodrígues-Bernal, Singular limit for a nonlinear parabolic equation terms concentrating in the boundary, J. of Math. Appl. 379 (2) (2011) 567-588.

[2] A. Jiménez-Casas and A. Rodrígues-Bernal, Asymptotic behavior of a parabolic problem with terms concentrating at the boundary, Nonlinear Analysis: Theory, Math. Appl. 71 (12) (2009) 237-2383.

[3] D. Blanchard, A. Gaudiello and G. Griso, Junction of a periodic family of elastic rods with a thin plate. Part II, J. Math. Pures et Appl. 88 (2) (2007) 149-190.

[4] D. Blanchard, A. Gaudiello and J. Mossino, Highly oscillating boundaries and redution of dimension: the critical case, Anal. Appl. 5 (2) (2007) 137-163.

[5] D. Cioranescu e P. Donato, An Introdution to Homogenization, Oxford Lecture Series in Mathematics and its application 17, Oxford press 1999.

[6] D. Henry, Geometry Theory of Semilinear Parabolic Equations, in Lectures Notes in Mathematics, vol. 840, Springer-Verlag, Berlin 1981.

[7] E. H. Rothe, Introduction to Various Aspects of Degree Theory in Banach Spaces, Math Surveys 23, American Math. Soc. Providence 1986.

[8] G. S. Aragão, A. L. Pereira and M. C. Pereira, Attractors for a nonlinear parabolic with terms concentrating on the boundary, Journal of Dynamics and Differential Equation, v. 26(4) (2014) 871-888.

[9] G. S. Aragão, A. L. Pereira and M. C. Pereira, A nonlinear eliptic problem with Terms concentrating in the boundary, Math. Methods Appl. Sci. 35 (9) (2012) 1110-1116.

[10] G. Folland, Introdution to Partial Diferential Equation, New Jersey, second edition 1995. 
[11] G. Raugel Dynamics of partial differential equations on thin domains, Lecture Notes in Math. 1609, Springer Verlag (1995).

[12] G. Vainikko, Approximative Methods for nonlinear equations (two approaches to the convergence problem), Nonlinear Analysis \& Application, 2 no 6 (1978) 647-687.

[13] H. Brezis, Funtional Analysis, Sobolev Spaces and Partial Diferential Equation, USA, Springer 2010.

[14] I. Fonseca and W. Gangbo, Degree Theory in Analysis and Applications, Oxford Lecture Series in Analysis and its Application.2, Clarendon Press. Oxford 1995.

[15] J. M. Arrieta, A. Jiménez-Casas e A. Rodrígues-Bernal, Flux terms and Robin boundary conditions as limits of reactions and potencials concentrating at the boundary, Revista Matemática Iberoamericana 24 (1) (2008) 183-221.

[16] J. M. Arrieta and S. M. Bruschi, Rapidly varying boundaries in equations with nonlinear boundary conditions. The case of a Lipschtz deformation, Math. Models and Meth. in Appl. Sciences 17 (10) (2007) 1555-1585.

[17] J. K. Hale and G. Raugel, Reaction-difusion equation on thin domain, J. Math. Pures et Appl. (9) 71 (1) (1992) 33-95.

[18] J. M. Arrieta, A. N. Carvalho, M. C. Pereira and R. P. Silva, Semilinear parabolic problems in thin domains with a highly oscillatory boundary, Nonlinear Analysis: Th. Math. Appl. 74 (2011) 5111-5132.

[19] J. M. Arrieta and M. C. Pereira, The Neumann problem in thin domains with very highly oscilatory boundaries, J. Math. Anal. Appl. 404 (2013) 86-104.

[20] J. M. Arrieta and M. C. Pereira, Homogenization in a thin domain with an oscillatory boundary, J. Math. Pures et Appl 96 (2011) 29-57.

[21] J. M. Arrieta and M. C. Pereira, Elliptic problems in thin domains with highly oscillanting boundaries, Bol. Soc. Esp. Mat. Apl. 51 (2010) 17-25

[22] K. Deimling, Nonlinear Functional Analysis, Springer-Verlag, New York 1984.

[23] M. A. Krasnoselskii and P. P. Zabreiko, Geometrical Methods of Nonlinear Analysis, Springer-Velag, New York 1984. 
[24] M. C. Pereira, Parabolic problems in highly oscillating thin domains, Annali di Matematica Pura ed Applicata (2014) DOI 10.1007/s10231-014-0421-7

[25] M. C. Pereira, Remarks on semilinear parabolic systems with terms concentrating in the boundary, Nonlinear Analysis Series B: Real World Applications 14 (4) (2013) 192101930.

[26] M. C. Pereira and R. P. Silva, Correctors for the Neumann problem in thin domain with locally periodic oscillatory structures, to apper in Quart. Appl. Math. (2014)

[27] M. C. Pereira and R. P. Silva, Error estimates for a Neumann problem in highly oscillating thin domains, Discrete and continuos Dyn. Systems 33 (2) (2013) 803-817.

[28] M. M. Cavalcanti e V. N. Cavalvanti, Introdução à Teoria das Distribuições e aos Espaços de Sobolev, Eduem Maringá- Paraná 2009.

[29] M. Prizzi, M. Rinaldi and K. P. Rybakowski, Curved thin domain and parabolic equations, Studia Mathematica 151 (2) (2002) 109-140

[30] M. Prizzi and K. P. Rybakowski, The effect of domain squeezing upon the dinamics of reaction-diffusion equations, J. of Diff. Equations 173 (2) (2001) 271-320 .

[31] P. Grivard, Elliptic Problems in Nonsmooth Domains, Pitman Advanced Publishing Program, 1985.

[32] R. A. Adams e J. J. F. Fournier, Sobolev Spaces, Second Edition, Academic Press, 2003.

[33] R. P. Silva, Behavior of the p-laplacian on thin domains, International J. of Diff. Eq 2013(2013).

[34] T. A. Mel'nyk and A. V. Popov, Asymptotic anaysis of boundary value and spectral problems in thin perforated domains with rapidly changing thikness and different limiting dimensions, Mat. Sb. 203 (8) (2012) 97-124.

[35] T. Elsken, Continuity of attractores for net-shaped on thin domain, J. Math. Pures et Appl. (9) 71 (1) (1992) 33-95.

[36] T. Kato, Perturbation Theory for Linear Operator, second ed., Grundlehren Math. Wiss., vol. 332, Springer, Berlim 1976.

[37] W. Rudin. Real and Complex Analysis, McGraw Hill, 1987. 